Universidade de São Paulo

Instituto de Física

\title{
Estudo de Emaranhamento num Sistema de Partículas Carregadas em Campo de Onda Plana Quantizada
}

\author{
Bruno Lima de Souza
}

Dissertação de mestrado apresentada ao

Instituto de Física para a obtenção do título de Mestre em Ciências

Orientador: Prof. Dr. Dmitri M. Gitman (IFUSP)

Banca examinadora:

Prof. Dr. Dmitri M. Gitman (IFUSP)

Prof. Dr. Arnaldo Gammal (IFUSP)

Prof. Dr. Dmitry Vasilevich (UFABC)

São Paulo, Setembro de 2012 
FICHA CATALOGRÁFICA

Preparada pelo Serviço de Biblioteca e Informação do Instituto de Física da Universidade de São Paulo

Souza, Bruno Lima de

Estudo de emaranhamento num sistema de partículas carregadas em campo de onda plana quantizada. São Paulo, 2012.

Dissertação (Mestrado) - Universidade de São Paulo. Instituto de Física, Depto. Física Nuclear.

Prientador: Prof. Dr. Dmitri Maximovitch Gitman

Área de Concentração: Física

Unitermos: 1. Física; 2. Mecânica Quântica; 3. Informação Quântica 


\section{Resumo}

Neste trabalho estudamos as propriedades de emaranhamento dos estados de dois quasifótons de frequências diferentes, fazendo a aproximação de que o vácuo de quasifótons é igual ao vácuo de fótons, no caso em que não temos campo externo algum e no caso da presença de um campo magnético externo, constante e homogêneo. Estudamos também as propriedades de emaranhamento do próprio vácuo de quasifótons e dos estados de dois quasifótons no caso monocromático, sem campo externo e com o vácuo de quasifótons levado em conta exatamente. 


\begin{abstract}
In this work we study the properties of entanglement of the states of two quasi-photons of different frequencies, considering the approximation that the quasi-photon's vacuum is equal the photon's vacuum, in the case where we have no external field and in the case where we have an external, constant and homogeneous magnetic field. We study also the properties of entanglement of the quasi-photon's vacuum and of the states of two quasi-photons in the monochromatic case, without external field and with the quasi-photon's vacuum considered exactly.
\end{abstract}


Dedico este trabalho aos meus pais

Pedro Bertolino de Souza

Maria Senhora Lima dos Santos Souza

e ao meu irmão

Daniel Lima de Souza 


\section{Agradecimentos}

Ao meu orientador professor Dr. Dmitri M. Gitman, ao professor Dr. João Carlos Alves Barata pelas discussões e pelo apoio e a todos os professores do Instituto de Física da USP que contribuíram para a minha formação.

Ao Mário César Baldiotti e ao João Luís Meloni Assirati pelas discussões, pelo apoio, pela motivação e pela amizade.

Aos meus amigos Gabriel Chicca Santucci, Cedrick Miranda Mello e Felipe Villaverde pelas várias discussões e pela amizade.

Aos meus amigos João Pedro Jericó de Andrade, André Antônio Monteiro Manoel, Paulo Victor Rossi, Petre Candelero Griscenco, Lucas Kaufmann Sacchetto, Henrique Meretti Camargo, Daniel Rutkowski Soler e mais todos aqueles com quem tenho o prazer de passar meus dias.

À FAPESP pelo suporte financeiro. 


\section{Sumário}

1 Introdução 1

2 Partículas Carregadas em Campo de Onda Plana Quantizada 4

2.1 Introdução . . . . . . . . . . . . . . . . . . 4

2.2 Solução Exata do Problema . . . . . . . . . . . . . . . . . . . . 11

2.2.1 Separação das Variáveis Espaciais e de Fótons . . . . . . . . . . . 11

2.2.2 Separação de Variáveis de Spin na Equação Fotônica $\ldots . . .16$

2.2 .3 Solução da Equação de Klein-Gordon . . . . . . . . . . . . . . . . 23

2.2 .3 .1 Redução à Forma Normal . . . . . . . . . . . . . . 23

2.3 Solução Exata em Presença de um Campo Magnético Externo Constante e Homogêneo . . . . . . . . . . . . . . . . . . . . . 28

3 Emaranhamento em Sistemas Bipartidos de Dimensão Finita 30

3.1 Formalismo da Matriz Densidade . . . . . . . . . . . . . . . . . 30

3.1.1 Propriedades Gerais da Matriz Densidade . . . . . . . . . . . . . 31

3.1 .2 A Matriz Densidade Reduzida . . . . . . . . . . . . . . . . 33

3.1 .3 A Distribuição de Wigner . . . . . . . . . . . . . . . . . 34

3.2 Critério de Separabilidade . . . . . . . . . . . . . . . 35

3.2.1 Estados Puros Bipartidos em Dimensão Finita: Decomposição de Sch-

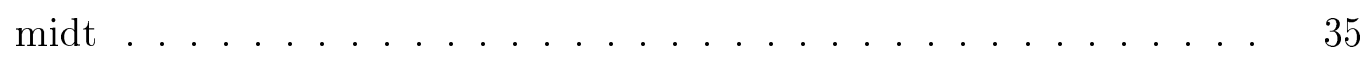

3.3 Quantificação do Emaranhamento e a Entropia de von Neumann . . . . . . . 41

3.4 Aplicação de Interesse . . . . . . . . . . . . . . . . . . . 43

3.5 Estados Puros Bipartidos em Dimensão Infinita: Critério de Peres-Horodecki-

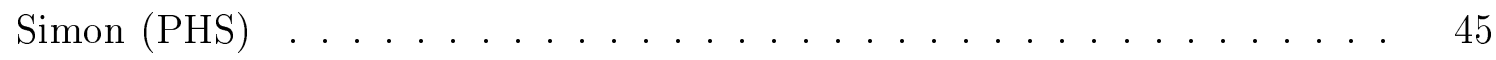

4 Emaranhamento de Quasifótons $\quad \mathbf{5 0}$

4.1 Emaranhamento com Vácuo de Quasifótons Aproximado . . . . . . . . 50

4.1.1 Estados de Dois Quasifótons com Vácuo de Quasifótons Aproximado 51 
4.1.2 Estado de Dois Quasifótons na Presença de Campo Magnético Externo 53

4.2 Emaranhamento com Vácuo de Quasifótons Exato . . . . . . . . . 56

4.2.1 Emaranhamento de Estados de Dois Quasifótons . . . . . . . . . 61

5 Conclusão $\quad 66$

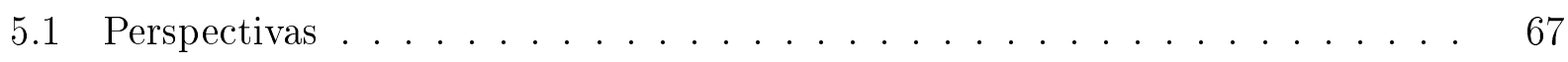

$\begin{array}{ll}\text { A Algumas Definições de Interesse } & 68\end{array}$

A.1 Soma Direta de Espaços de Hilbert . . . . . . . . . . . . . . 68

A.2 Produto Tensorial de Espaços de Hilbert . . . . . . . . . . . . . . 68

A.3 Produto Tensorial de Operadores de Espaços de Hilbert . . . . . . . . . . . 69

A.4 Espaço de Fock . . . . . . . . . . . . . . . . . 69

$\begin{array}{ll}\text { B Transformações Canônicas Lineares } & 70\end{array}$

$\begin{array}{ll}\text { C Sistemas com Hamiltonianos Quadráticos } & 73\end{array}$

$\begin{array}{ll}\text { D Representação de Fock-Bargmann } & 77\end{array}$

E Um Fato Sobre Estados Comprimidos $\quad \mathbf{8 1}$

E.1 Fatoração do Operador de Compressão . . . . . . . . . . . . . . . 81

F O Vácuo de Quasifótons em Termos dos Operadores de Compressão de duas Polarizações e de Deslocamento 


\section{Capítulo 1}

\section{Introdução}

Desde os trabalhos pioneiros de E. Schrödinger [22], estados emaranhados possuem um destaque especial, uma vez que apresentam comportamentos sem análogo clássico. Tais estados são o cerne dos trabalhos da chamada informação e computação quântica e os novos paradigmas impostos por estas teorias colocam os estados emaranhados no centro de interesses da física do século XXI. Esses estados nos permitem, de forma segura, trocar chaves criptográficas e distribuir informação quântica via teletransporte [10] e desempenham um papel fundamental nas mais importantes aplicações da computação quântica [18]. Deste modo, é possível resolver problemas que não são tratáveis com a computação clássica [8], como por exemplo, a decomposição em fatores primos [23] e a simulação de outros sistemas quânticos [13].

Uma questão fundamental para a computação quântica é a produção controlada de emaranhamento. Mostramos neste trabalho que é possível produzir fótons emaranhados através da interação com elétrons e campos eletromagnéticos.

Nos anos 70, V. Bagrov, P. V. Bozrikov, D. M. Gitman, V. A. Kuchin e M. M. Lavrov encontraram e analisaram soluções exatas para as equações de Dirac e Klein-Gordon em presença de uma campo eletromagnético quantizado do tipo onda-plana. Estas soluções e suas propriedades foram largamente discutidas numa série de artigos [1, 2, 3, 4, 5] e os resultados principais foram compilados num capítulo do livro entitulado "Exact Solutions of Relativistic Wave Equations" [7].

Estes resultados podem ser sintetizados, de forma simplificada, da seguinte maneira. Considere a eletrodinâmica quântica, a teoria que nos diz como se dá a interação entre o campo eletromagnético e um campo espinorial que descreve elétrons e pósitrons. Esta teoria pode ser escrita nos mesmos termos da mecânica quântica, i.e. podemos escrever uma equação de Schrödinger para ela

$$
i \hbar \frac{\partial}{\partial t}|\Psi\rangle=H_{Q E D}|\Psi\rangle
$$


onde $H_{Q E D}$ é o Hamiltoniano da eletrodinâmica quântica e $|\Psi\rangle$ contém toda a informação necessária para descrever o sistema, ou seja, em $|\Psi\rangle$ está contida a informação sobre quais partículas estão presentes no sistema, como elas evoluem no tempo, quais são criadas e quais são aniquiladas. Nos trabalhos acima citados foi analisado o setor de um elétron dessa teoria, i.e. a "projeção" da equação (1.0.1) sobre os estados $|\Phi\rangle$ que contém apenas um elétron e um número arbitrário de fótons, ou seja, foram ignorados estados com mais elétrons ou mesmo estados com pósitrons. De fato, esses autores perceberam que na equação de movimento obtida a partir de (1.0.1) pela projeção aparecem contribuições provenientes de estados com mais elétrons e pósitrons, porém essas contribuições vão a zero quando restringimos o campo eletromagnético a ter apenas modos que se propagam numa certa direção, i.e. ao nos restringirmos à situação em que temos apenas fótons viajando numa certa direção. Para tal equação, sem as contribuições vindas de estados com mais elétrons e pósitrons, foram encontradas soluções exatas.

Essas soluções são dadas em termos dos autoestados de um hamiltoniano quadrático nos operadores de criação e aniquilação de fótons, de forma que tais autoestados não têm número definido de fótons mas sim de uma certa quasipartícula que chamamos de quasifóton. Essas soluções nos trazem informações sobre como partículas carregadas alteram o estado de fótons.

Nosso objetivo nessa dissertação é discutir propriedades de emaranhamento desses estados de número definido de quasifótons. Em outras palavras, estamos preocupados em saber se a interação eletromagnética entre as partículas carregadas e os fótons é capaz de emaranhar tais fótons.

O capítulo 4 contém os resultados do nosso trabalho. Nesse capítulo analisamos as propriedades de emaranhamento de estados de dois quasifótons de frequências e polarizações distintas escritos em termos de fótons e mostramos que, fazendo a aproximação de que o vácuo de quasifótons é igual ao vácuo de fótons, temos emaranhamento entre as frequências dos fótons se adicionarmos ao sistema um campo magnético externo, constante e homogêneo na direção de propagação dos fótons. Mostramos também que se considerarmos estados de dois quasifótons de mesma frequência, porém de polarizações distintas, levando em conta o vácuo de quasifótons exatamente, existe correlação entre número de fótons em cada estado de polarização. Analisamos para algumas frequências do campo eletromagnético como a quantidade de emaranhamento do sistema muda em termos do momento do elétron e encontramos um fenômeno curioso de oscilação da quantidade de emaranhamento que pode ser apreciado na figura 4.2.1.

Para obtermos os resultados do capítulo 4 é necessário conhecer a física do sistema eletrodinâmico proposto e elementos da teoria do emaranhamento, que serão objeto dos capítulos 2 e 3. 
No capítulo 2, fazemos uma revisão das soluções exatas apresentadas em [1, 2, 3, 4, 5] que é o elemento básico na construção dos estados emaranhados discutidos acima. Apresentamos as soluções na ausência de campos externos e como elas são modificadas pela presença de um campo magnético externo constante e homogêneo na direção de propagação dos fótons.

No capítulo 3 , fazemos a revisão de alguns resultados básicos sobre emaranhamento em sistemas bipartidos puros. Para o caso de dimensão finita, apresentamos um critério geral de separabilidade, a decomposição de Schmidt e apresentamos a entropia de von Neumann dos subsistemas como um quantificador de emaranhamento. Para o caso de dimensão infinita discutimos dois critérios de separabilidade, o critério PPT proposto por A. Peres em [20], que é apenas uma condição suficiente para separabilida, e o critério PHS que é um refinamento do critério PPT proposto por Horodecki em [17]. 


\section{Capítulo 2}

\section{Partículas Carregadas em Campo de Onda Plana Quantizada}

\subsection{Introdução}

Seja $H$ o Hamiltoniano da eletrodinâmica quântica e $|\Psi(t)\rangle$ um estado que descreve os campos eletromagnético e espinorial. A evolução temporal desse estado é dada pela equação de Schrödinger

$$
i \partial_{t}|\Psi(t)\rangle=H|\Psi(t)\rangle .
$$

Formalmente, $|\Psi(t)\rangle \in \mathcal{F}_{\text {total }}=\mathcal{F}_{\text {esp }} \otimes \mathcal{F}_{\text {em }}{ }^{*}$, onde $\mathcal{F}_{\text {esp }}$ é o espaço de Fock associado à parte espinorial do sistema e $\mathcal{F}_{\text {em }}$ o espaço de Fock associado à parte eletromagnética. Seja $\left\{\left|\psi_{i}\right\rangle\right\}$ uma base de $\mathcal{F}_{\text {esp }}$ e $\left\{\left|\phi_{j}\right\rangle\right\}$ uma base para $\mathcal{F}_{\text {em }}$. É natural que todo estado $|\Psi(t)\rangle \in \mathcal{F}_{\text {total }}$ possa ser colocado na forma:

$$
|\Psi(t)\rangle=\sum_{i, j} a_{i j}(t)\left|\psi_{i}\right\rangle \otimes\left|\phi_{j}\right\rangle, \quad a_{i j} \in \mathbb{C}
$$

Por outro lado, podemos reescrever a (2.1.2) na forma:

$$
|\Psi(t)\rangle=\sum_{i}\left|\psi_{i}\right\rangle \otimes\left(\sum_{j} a_{i j}(t)\left|\phi_{j}\right\rangle\right) \equiv \sum_{i}\left|\psi_{i}\right\rangle \otimes\left|\varphi_{i}(t)\right\rangle .
$$

Podemos perceber que o estado $\left|\varphi_{i}(t)\right\rangle \in \mathcal{F}_{\text {em }}$ é completamente determinado por $|\Psi(t)\rangle \in \mathcal{F}$ e por $\left|\psi_{i}\right\rangle \in \mathcal{F}_{\text {esp }}$. De fato, considere o seguinte projetor:

$$
\mathcal{P}_{\left|\psi_{j}\right\rangle}=\left|\psi_{j}\right\rangle\left\langle\psi_{j}\right| \otimes \mathbb{I}
$$

${ }^{*}$ Ver apêndice A para definições gerais sobre produtos tensoriais, traços parciais, espaço de Fock e etc. 
A ação desse projetor sobre $|\Psi\rangle$ é dada por:

$$
\mathcal{P}_{\left|\psi_{j}\right\rangle}|\Psi(t)\rangle=\left|\psi_{j}\right\rangle \otimes\left|\varphi_{j}(t)\right\rangle
$$

Utilizando essa expressão, podemos construir a matriz densidade associada ao estado $\mathcal{P}_{\left|\psi_{j}\right\rangle}|\Psi(t)\rangle$ e tomar seu traço parcial em relação ao subsistema espinorial, i.e. $\mathcal{F}_{\text {esp }}$, e obtemos a matriz densidade reduzida associada ao estado puro $\left|\varphi_{j}(t)\right\rangle$. Esse procedimento nos mostra que de fato podemos determinar $\left|\varphi_{i}\right\rangle$ a partir de $|\Psi(t)\rangle$ e $\left|\psi_{i}\right\rangle$.

Para simplificar a notação, ao invés de usar $\left\{\left|\psi_{i}\right\rangle\right\}$ para $\mathcal{F}_{\text {esp }}$, usaremos $\{|k\rangle\}$, onde os estados $|k\rangle, k \in \mathbb{N}$, representam de forma genérica um estado com $n$ pósitrons e $m$ elétrons, de forma que $k=n+m$. Os operadores associados ao campo espinorial são

$$
\begin{aligned}
\psi(\boldsymbol{x}) & =\int \frac{d^{3} \boldsymbol{p}}{(2 \pi)^{3}} \frac{1}{\sqrt{2 E_{\boldsymbol{p}}}} \sum_{s}\left(a_{\boldsymbol{p}}^{s} u^{s}(\boldsymbol{p}) e^{-i \boldsymbol{p} \cdot \boldsymbol{x}}+b_{\boldsymbol{p}}^{s \dagger} v^{s}(\boldsymbol{p}) e^{i \boldsymbol{p} \cdot \boldsymbol{x}}\right) \\
\bar{\psi}(\boldsymbol{y}) & =\int \frac{d^{3} \boldsymbol{p}}{(2 \pi)^{3}} \frac{1}{\sqrt{2 E_{\boldsymbol{p}}}} \sum_{s}\left(b_{\boldsymbol{p}}^{s} v^{s}(\boldsymbol{p}) e^{-i \boldsymbol{p} \cdot \boldsymbol{y}}+a_{\boldsymbol{p}}^{s \dagger} \bar{u}^{s}(\boldsymbol{p}) e^{i \boldsymbol{p} \cdot \boldsymbol{y}}\right),
\end{aligned}
$$

onde $a_{p}^{s \dagger}, a_{p}^{s}$ e $b_{p}^{s \dagger}, b_{p}^{s}$ são, respectivamente, operadores de criação e aniquilação de elétrons e pósitrons. O estado $|k\rangle$ representa* a ação dos operadores do campo espinorial $\psi(\boldsymbol{x})$ e $\bar{\psi}(\boldsymbol{y})$ no vácuo, i.e.

$$
\psi\left(\boldsymbol{x}_{1}\right) \ldots \psi\left(\boldsymbol{x}_{n}\right) \bar{\psi}\left(\boldsymbol{y}_{1}\right) \ldots \bar{\psi}\left(\boldsymbol{y}_{m}\right)|0\rangle
$$

e em particular

$$
\left|1_{\boldsymbol{x}}\right\rangle=\bar{\psi}(\boldsymbol{x})|0\rangle
$$

representa o estado de um elétron ${ }^{\dagger}$. Usando (2.1.1) e (2.1.3) obtemos:

$$
i \partial_{t}\left(\sum_{k=0}^{\infty}|k\rangle \otimes\left|\varphi_{k}(t)\right\rangle\right)=H\left(\sum_{k=0}^{\infty}|k\rangle \otimes\left|\varphi_{k}(t)\right\rangle\right)
$$

Usando a forma explícita de $\hat{H}$, i.e.

$$
H=\int d^{3} \boldsymbol{x} \mathscr{H}(\boldsymbol{x})=\int d^{3} \boldsymbol{x}\left[\mathbb{I}(\boldsymbol{x}) \otimes \mathscr{H}_{\gamma}(\boldsymbol{x})+\mathscr{H}_{D}(\boldsymbol{x}) \otimes \mathbb{I}(\boldsymbol{x})+\mathscr{H}_{I}\right]
$$

\footnotetext{
*Claro que essa notação é ambígua pois vários estados diferentes estão sendo representados pelo mesmo símbolo, porém a notação será útil para nosso uso e a ambiguidade não nos incomodará.

†O índice $\boldsymbol{x}$ colocado no estado $\left|1_{\boldsymbol{x}}\right\rangle$ serve para recordar a dependência desse estado das coordenadas do espaço. Analogamente, deveríamos colocar $k$ índices similares no estado $|k\rangle$, porém não o faremos para não sobrecarregar a notação.
} 


$$
\begin{aligned}
H_{D} & =\int d^{3} \boldsymbol{x} \mathscr{H}_{D}(\boldsymbol{x})=\int d^{3} \boldsymbol{x} \quad \bar{\psi}(\boldsymbol{x})(-i \boldsymbol{\gamma} \cdot \nabla+m) \psi(\boldsymbol{x}), \\
H_{I} & =\int d^{3} \boldsymbol{x} \mathscr{H}_{I}(\boldsymbol{x})=\int d^{3} \boldsymbol{x} \quad e \bar{\psi}(\boldsymbol{x}) \gamma_{\mu} \psi(\boldsymbol{x}) A^{\mu}(x)
\end{aligned}
$$

onde $\mathbb{I}(\boldsymbol{x})$ é o operador identidade no ponto $\boldsymbol{x}$, e é a carga elétrica e $A^{\mu}(x)$ é o quadripotencial eletromagnético, a equação (2.1.4) se torna:

$$
\begin{aligned}
\sum_{k=0}^{\infty}|k\rangle \otimes\left(i \partial_{t}\left|\varphi_{k}(t)\right\rangle\right)= & \sum_{k=0}^{\infty} \int d^{3} \boldsymbol{x}\left\{|k\rangle \otimes\left(\mathscr{H}_{\gamma}\left|\varphi_{k}(t)\right\rangle\right)+\left(\mathscr{H}_{D}|k\rangle\right) \otimes\left|\varphi_{k}(t)\right\rangle+\right. \\
& \left.+\left(e \bar{\psi}(\boldsymbol{x}) \gamma_{\mu} \psi(\boldsymbol{x})|k\rangle\right) \otimes\left(A^{\mu}(x)\left|\varphi_{k}(t)\right\rangle\right)\right\} .
\end{aligned}
$$

Considere o operador que projete sobre o espaço de $k^{\prime}$ férmions distribuídos pelo espaço, i.e.

$$
\int\left(\prod_{j=1}^{k^{\prime}} d^{3} \boldsymbol{y}_{j}\right)\left|k^{\prime}\right\rangle\left\langle k^{\prime}\right| \otimes \mathbb{I}
$$

Aplicando tal projetor em ambos os lados da última expressão, obtemos*:

$$
\begin{aligned}
\sum_{k=0}^{\infty}\left(\left|k^{\prime}\right\rangle\left\langle k^{\prime} \mid k\right\rangle\right) \otimes\left(i \partial_{t}\left|\varphi_{k}(t)\right\rangle\right)= & \sum_{k=0}^{\infty} \int d^{3} \boldsymbol{x}\left\{\left(\left|k^{\prime}\right\rangle\left\langle k^{\prime} \mid k\right\rangle\right) \otimes\left(\mathscr{H}_{\gamma}\left|\varphi_{k}(t)\right\rangle\right)+\right. \\
& +\left(\left|k^{\prime}\right\rangle\left\langle k^{\prime}\left|\mathscr{H}_{D}\right| k\right\rangle\right) \otimes\left|\varphi_{k}(t)\right\rangle+ \\
& \left.+\left(\left|k^{\prime}\right\rangle\left\langle k^{\prime}\left|e \bar{\psi}(\boldsymbol{x}) \gamma_{\mu} \psi(\boldsymbol{x})\right| k\right\rangle\right) \otimes\left(A^{\mu}(x)\left|\varphi_{k}(t)\right\rangle\right)\right\} .
\end{aligned}
$$

Os termos $\left\langle k^{\prime} \mid k\right\rangle$ forçam $k=k^{\prime}$, de forma que

$$
\begin{aligned}
\left|k^{\prime}\right\rangle \otimes\left(i \partial_{t}\left|\varphi_{k^{\prime}}(t)\right\rangle\right)= & \left|k^{\prime}\right\rangle \otimes \int d^{3} \boldsymbol{x}\left\{\mathscr{H}_{\gamma}\left|\varphi_{k^{\prime}}(t)\right\rangle+\sum_{k=0}^{\infty}\left[\left\langle k^{\prime}\left|\mathscr{H}_{D}\right| k\right\rangle\left|\varphi_{k}(t)\right\rangle+\right.\right. \\
& \left.\left.+\left\langle k^{\prime}\left|e \bar{\psi}(\boldsymbol{x}) \gamma_{\mu} \psi(\boldsymbol{x})\right| k\right\rangle A^{\mu}(x)\left|\varphi_{k}(t)\right\rangle\right]\right\} .
\end{aligned}
$$

*A integração em $d^{3} \boldsymbol{y}_{j}$ será omitida para não sobrecarregarmos a notação, porém não podemos esquecer de sua existência. Note que existem $k^{\prime}$ integrações em $d^{3} \boldsymbol{y}_{j}$ pois cada uma está associada a uma das $k^{\prime}$ partículas do estado $\left|k^{\prime}\right\rangle$. 
Como a última expressão deve ser verdadeira para todo vetor $\left|k^{\prime}\right\rangle$, então:

$$
\begin{aligned}
i \partial_{t}\left|\varphi_{k^{\prime}}(t)\right\rangle=\int d^{3} \boldsymbol{x}\left\{\mathscr{H}_{\gamma}\left|\varphi_{k^{\prime}}(t)\right\rangle+\right. \\
\left.\quad+\sum_{k=0}^{\infty}\left[\left\langle k^{\prime}\left|\mathscr{H}_{D}\right| k\right\rangle\left|\varphi_{k}(t)\right\rangle+\left\langle k^{\prime}\left|e \bar{\psi}(\boldsymbol{x}) \gamma_{\mu} \psi(\boldsymbol{x})\right| k\right\rangle A^{\mu}(x)\left|\varphi_{k}(t)\right\rangle\right]\right\} .
\end{aligned}
$$

Considerando o caso $k^{\prime}=1$, a equação (2.1.6) se torna:

$$
\begin{aligned}
i \partial_{t}\left|\varphi_{1}(t)\right\rangle=\int d^{3} \boldsymbol{x} & \left\{\mathscr{H}_{\gamma}\left|\varphi_{1}(t)\right\rangle+\right. \\
& \left.+\sum_{k=0}^{\infty}\left[\left\langle 1\left|\mathscr{H}_{D}\right| k\right\rangle\left|\varphi_{k}(t)\right\rangle+\left\langle 1\left|e \bar{\psi}(\boldsymbol{x}) \gamma_{\mu} \psi(\boldsymbol{x})\right| k\right\rangle A^{\mu}(x)\left|\varphi_{k}(t)\right\rangle\right]\right\} .
\end{aligned}
$$

De fato, estamos interessados nos termos com $k=0$ e $k=1$ da equação (2.1.7), todo o resto será denotado por $\Delta$. Explicitando os termos de interesse, chegamos em:

$$
\begin{aligned}
i \partial_{t}\left|\varphi_{1}(t)\right\rangle=\int d^{3} \boldsymbol{x} d^{3} \boldsymbol{y}\left[\mathscr{H}_{\gamma}\left|\varphi_{1}(t)\right\rangle+\left\langle 1_{\boldsymbol{y}}\left|\mathscr{H}_{D}\right| 0\right\rangle\left|\varphi_{0}(t)\right\rangle+\left\langle 1_{\boldsymbol{y}}\left|e \bar{\psi}(\boldsymbol{x}) \gamma_{\mu} \psi(\boldsymbol{x})\right| 0\right\rangle A^{\mu}(x)\left|\varphi_{0}(t)\right\rangle\right. \\
\left.+\left\langle 1_{\boldsymbol{y}}\left|\mathscr{H}_{D}\right| 1\right\rangle\left|\varphi_{1}(t)\right\rangle+\left\langle 1_{\boldsymbol{y}}\left|e \bar{\psi}(\boldsymbol{x}) \gamma_{\mu} \psi(\boldsymbol{x})\right| 1\right\rangle A^{\mu}(x)\left|\varphi_{1}(t)\right\rangle\right]+\Delta, \quad \text { (2.1.8) } \\
\Delta=\sum_{k=2}^{\infty}\left\langle 1\left|e \bar{\psi}(\boldsymbol{x}) \gamma_{\mu} \psi(\boldsymbol{x})\right| k\right\rangle A^{\mu}(x)\left|\varphi_{k}(t)\right\rangle, \quad\left\langle 1\left|\hat{\mathscr{H}}_{D}\right| k\right\rangle=0, k \neq 1 .
\end{aligned}
$$

Note que explicitamos na expressão (2.1.8) a integração em $d^{3} \boldsymbol{y}$ proveniente do projetor (2.1.5). Prontamente concluímos que os termos $\left\langle 1\left|\mathscr{H}_{D}\right| 0\right\rangle$ e $\left\langle 1\left|e \bar{\psi}(\boldsymbol{x}) \gamma_{\mu} \psi(\boldsymbol{x})\right| 0\right\rangle$ são nulos porque nem a Hamiltoniana livre de Dirac nem a Hamiltoniana de interação acoplam o vácuo com o estado de um elétron, ou seja, essas transições não são possíveis e isso é devido à conservação de carga. Observe que há amplitudes de transições em $\Delta$ que são nulas pelo argumento de conservação de carga, no entanto existem termos que conservam carga e que devem contribuir. Nos resta calcular $\left\langle 1\left|\mathscr{H}_{D}\right| 1\right\rangle$ e $\left\langle 1\left|e \bar{\psi}(\boldsymbol{x}) \gamma_{\mu} \psi(\boldsymbol{x})\right| 1\right\rangle$, lembrando que os campos $\psi(\boldsymbol{x})$ e $\bar{\psi}(\boldsymbol{y})$ satisfazem a seguinte relação de anticomutação:

$$
\{\psi(\boldsymbol{x}), \bar{\psi}(\boldsymbol{y})\}=\gamma_{0} \delta^{3}(\boldsymbol{x}-\boldsymbol{y}) \Rightarrow \nabla_{\boldsymbol{x}}\{\psi(\boldsymbol{x}), \bar{\psi}(\boldsymbol{y})\}=\left\{\nabla_{\boldsymbol{x}} \psi(\boldsymbol{x}), \bar{\psi}(\boldsymbol{y})\right\}=\gamma_{0} \nabla_{\boldsymbol{x}} \delta^{3}(\boldsymbol{x}-\boldsymbol{y})
$$


1. $\int d^{3} \boldsymbol{y}\left\langle 1_{\boldsymbol{y}}\left|\mathscr{H}_{D}\right| 1_{\boldsymbol{z}}\right\rangle$ :

$$
\begin{aligned}
\int d^{3} \boldsymbol{y}\left\langle 1_{\boldsymbol{y}}\left|\mathscr{H}_{D}\right| 1_{\boldsymbol{z}}\right\rangle= & \int d^{3} \boldsymbol{y}\left\langle 0\left|\psi(\boldsymbol{y}) \bar{\psi}(\boldsymbol{x})\left(-i \boldsymbol{\gamma} \cdot \nabla_{\boldsymbol{x}}+m\right) \psi(\boldsymbol{x}) \psi^{\dagger}(\boldsymbol{z})\right| 0\right\rangle \\
= & \int d^{3} \boldsymbol{y}\langle 0|\left[\gamma_{0} \delta^{3}(\boldsymbol{x}-y)-\bar{\psi}(\boldsymbol{x}) \psi(\boldsymbol{y})\right] \times \\
& \times\left(-i \boldsymbol{\gamma} \cdot \nabla_{\boldsymbol{x}}+m\right)\left[\gamma_{0} \delta^{3}(\boldsymbol{x}-\boldsymbol{z})-\bar{\psi}(\boldsymbol{z}) \psi(\boldsymbol{x})\right] \gamma_{0}|0\rangle \\
= & \int d^{3} \boldsymbol{y}\left[m \gamma_{0}-i \boldsymbol{\alpha} \cdot\left(\nabla_{\boldsymbol{x}} \delta^{3}(\boldsymbol{x}-\boldsymbol{z})\right)\right] \delta^{3}(\boldsymbol{x}-\boldsymbol{y}) \\
= & \int d^{3} \boldsymbol{y} \delta^{3}(\boldsymbol{x}-\boldsymbol{z})\left[m \gamma_{0}+i \boldsymbol{\alpha} \cdot\left(\nabla_{\boldsymbol{x}} \delta^{3}(\boldsymbol{x}-\boldsymbol{y})\right)\right] \\
\int d^{3} \boldsymbol{y}\left\langle 1_{\boldsymbol{y}}\left|\mathscr{H}_{D}\right| 1_{\boldsymbol{z}}\right\rangle= & \delta^{3}(\boldsymbol{x}-\boldsymbol{z})\left[m \gamma_{0}-i \boldsymbol{\alpha} \cdot \nabla_{\boldsymbol{x}}\right]
\end{aligned}
$$

2. $\int d^{3} \boldsymbol{y}\left\langle 1_{\boldsymbol{y}}\left|e \bar{\psi}(\boldsymbol{x}) \gamma_{\mu} \psi(\boldsymbol{x})\right| 1_{\boldsymbol{z}}\right\rangle$ :

$$
\begin{aligned}
\int d^{3} \boldsymbol{y}\left\langle 1_{\boldsymbol{y}}\left|e \bar{\psi}(\boldsymbol{x}) \gamma_{\mu} \psi(\boldsymbol{x})\right| 1_{\boldsymbol{z}}\right\rangle= & e \int d^{3} \boldsymbol{y}\left\langle 0\left|\psi(\boldsymbol{y}) \bar{\psi}(\boldsymbol{x}) \gamma_{\mu} \psi(\boldsymbol{x}) \psi^{\dagger}(\boldsymbol{z})\right| 0\right\rangle \\
= & e \int d^{3} \boldsymbol{y}\langle 0|\left[\gamma_{0} \delta^{3}(\boldsymbol{x}-y)-\bar{\psi}(\boldsymbol{x}) \psi(\boldsymbol{y})\right] \gamma_{\mu} \times \\
& \times\left[\gamma_{0} \delta^{3}(\boldsymbol{x}-\boldsymbol{z})-\bar{\psi}(\boldsymbol{z}) \psi(\boldsymbol{x})\right] \gamma_{0}|0\rangle \\
\int d^{3} \boldsymbol{y}\left\langle 1_{\boldsymbol{y}}\left|e \bar{\psi}(\boldsymbol{x}) \gamma_{\mu} \psi(\boldsymbol{x})\right| 1_{\boldsymbol{z}}\right\rangle= & e \gamma_{0} \gamma_{\mu} \delta^{3}(\boldsymbol{x}-\boldsymbol{z}) .
\end{aligned}
$$

Inserindo esses resultados em (2.1.8) e integrando em $\boldsymbol{x}$, temos:

$$
\begin{gathered}
i \partial_{t}\left|\varphi_{1}(t)\right\rangle=\left[H_{\gamma}+m \gamma_{0}-i \boldsymbol{\alpha} \cdot \nabla_{\boldsymbol{z}}+e \gamma_{0} \gamma_{\mu} A^{\mu}\right]\left|\varphi_{1}(t)\right\rangle+\Delta \\
\therefore i \partial_{t}\left|\varphi_{1}(t)\right\rangle=\left[H_{\gamma}+\boldsymbol{\alpha} \cdot \boldsymbol{P}+m \gamma_{0}\right]\left|\varphi_{1}(t)\right\rangle+\Delta, \\
\boldsymbol{P}=\boldsymbol{p}-e \boldsymbol{A}(\boldsymbol{x}), \boldsymbol{p}=-i \nabla, \boldsymbol{\alpha}=\gamma_{0} \boldsymbol{\gamma} .
\end{gathered}
$$

O termo $\Delta$ em (2.1.9) só contém amplitudes envolvendo mais de uma partícula fermiônica. Na equação (2.1.9) estamos usando o gauge de Coulomb, onde $A^{0}=0$ e $\nabla \cdot \boldsymbol{A}=0$. No que segue, passaremos a denotar $\left|\varphi_{1}(t)\right\rangle$ simplesmente por $\Psi(x)^{*}$ e portanto a equação (2.1.9) se reescreve como:

$$
i \partial_{t} \Psi(x)=\left[H_{\gamma}+\boldsymbol{\alpha} \cdot \boldsymbol{P}+m \gamma_{0}\right] \Psi(x)+\Delta .
$$

${ }^{*}$ O fato de denotarmos $\left|\varphi_{1}(t)\right\rangle$ por $\Psi(x)$ sugere que estamos olhando uma representação do vetor $\left|\varphi_{1}(t)\right\rangle$ num espaço de funções, porém isso não é verdade. A notação $\Psi(x)$ é utilizada simplesmente para evidenciarmos a dependência em $x$ que ocorre no estado $\left|\varphi_{1}(t)\right\rangle$ e também para simplificar a notação. 
Podemos escrever $\hat{H}_{\gamma}$ e $\boldsymbol{A}(\boldsymbol{x})$ em termos dos operadores de criação e aniquilação de fótons de momento $\boldsymbol{k}$ e polarização $\lambda, a_{\boldsymbol{k}, \lambda}^{\dagger}$ e $a_{\boldsymbol{k}, \lambda}$ :

$$
\begin{aligned}
H_{\gamma} & =\sum_{\boldsymbol{k}, \lambda}|\boldsymbol{k}| a_{\boldsymbol{k}, \lambda}^{\dagger} a_{\boldsymbol{k}, \lambda} \\
\boldsymbol{A}(\boldsymbol{x}) & =\sum_{\boldsymbol{k}, \lambda} \frac{1}{\sqrt{2 \omega_{k} V}}\left[a_{\boldsymbol{k}, \lambda} \boldsymbol{e}_{\lambda}(\boldsymbol{k}) e^{i \boldsymbol{k} \cdot \boldsymbol{x}}+a_{\boldsymbol{k}, \lambda}^{\dagger} \boldsymbol{e}_{\lambda}^{*}(\boldsymbol{k}) e^{-i \boldsymbol{k} \cdot \boldsymbol{x}}\right],
\end{aligned}
$$

onde $V$ é o volume de quantização e os $\boldsymbol{e}_{\lambda}(\boldsymbol{k})$ são vetores de polarização que satisfazem as seguintes propriedades:

$$
\begin{gathered}
\boldsymbol{e}_{\lambda}(\boldsymbol{k}) \cdot \boldsymbol{k}=\boldsymbol{e}_{\lambda}^{*}(\boldsymbol{k}) \cdot \boldsymbol{k}=0, \lambda= \pm 1 \\
\boldsymbol{e}_{\lambda}(\boldsymbol{k}) \cdot \boldsymbol{e}_{\lambda^{\prime}}^{*}(\boldsymbol{k})=\delta_{\lambda, \lambda^{\prime}}
\end{gathered}
$$

Essa escolha de vetores de polarização garante que o potencial $\boldsymbol{A}(\boldsymbol{x})$ esteja no gauge de Coulomb, i.e.

$$
\nabla \cdot \boldsymbol{A}(\boldsymbol{x})=0
$$

Daqui pra frente iremos ignorar efeitos de criação de pares virtuais ou reais na (2.1.10), i.e. vamos desprezar o termo $\Delta$, com isso, nos resta a equação de Schrödinger para um único elétron interagindo com o campo eletromagnético quantizado. Escrevendo a (2.1.10) em representação de interação, temos:

$$
\begin{gathered}
\Phi(x)=e^{i H_{\gamma} t} \Psi(x) \Leftrightarrow \Psi(x)=e^{-i H_{\gamma} t} \Phi(x) \\
i \partial_{t} \Phi(x)=-H_{\gamma} e^{i H_{\gamma} t} \Psi(x)+e^{i H_{\gamma} t} i \partial_{t} \Psi(x) \\
=-H_{\gamma} \Phi(x)+e^{i H_{\gamma} t}\left[H_{\gamma}+\boldsymbol{\alpha} \cdot \boldsymbol{P}+m \gamma^{0}\right] e^{-i H_{\gamma} t} \Phi(x) \\
i \partial_{t} \Phi(x)=\left[\boldsymbol{\alpha} \cdot\left(\boldsymbol{p}-e e^{i H_{\gamma} t} \boldsymbol{A}(\boldsymbol{x}) e^{-i H_{\gamma} t}\right)+m \gamma^{0}\right] \Phi(x)
\end{gathered}
$$

o termo $e^{i H_{\gamma} t} \boldsymbol{A}(\boldsymbol{x}) e^{-i H_{\gamma} t}$ é o potencial em representação de interação e será denotado por $A^{\mu}(x)$

$$
\begin{gathered}
A^{\mu}(x)=\sum_{\boldsymbol{k}, \lambda} \frac{1}{\sqrt{2 \omega_{k} V}}\left[a_{\boldsymbol{k}, \lambda} e_{\lambda}^{\mu}(\boldsymbol{k}) e^{-i k x}+a_{\boldsymbol{k}, \lambda}^{\dagger} e_{\lambda}^{\mu *}(\boldsymbol{k}) e^{i k x}\right] \\
k^{\mu}=\left\{\omega_{k}, \boldsymbol{k}\right\}, k^{2}=0, k_{\mu} e_{\lambda}^{\mu}(\boldsymbol{k})=k_{\mu} e_{\lambda}^{\mu *}(\boldsymbol{k})=0, \\
e_{\lambda}^{\mu}(\boldsymbol{k})=\left\{0, \boldsymbol{e}_{\lambda}(\boldsymbol{k})\right\}, e_{\lambda \mu}^{*}(\boldsymbol{k}) e_{\lambda^{\prime}}^{\mu}(\boldsymbol{k})=-\delta_{\lambda, \lambda^{\prime}} .
\end{gathered}
$$


Multiplicando a (2.1.14) por $\gamma^{0}$,obtemos:

$$
\begin{gathered}
p_{0} \gamma^{0} \Phi(x)=\left[\boldsymbol{\gamma} \cdot\left(\boldsymbol{p}-e A^{\mu}(x)\right)+m\right] \Phi(x) \\
\therefore\left(\gamma^{\mu} P_{\mu}-m\right) \Phi(x)=0, P_{\mu}=i \partial_{\mu}-e A_{\mu}(x) .
\end{gathered}
$$

Assim, soluções da equação de Dirac (2.1.16) com o potencial (2.1.15) torna possível o estudo de problemas onde um único elétron interage com um número arbitrário de fótons, no entanto efeitos de criação de pares reais ou virtuais são desprezados. Infelizmente, mesmo com o termo $\Delta$ desprezado, o problema não apresenta nenhuma solução exata, de forma que somos obrigados a tratá-lo de forma perturbativa. É notável, entretanto, que existem situações onde a perturbação não é pequena em relação ao problema livre. Por exemplo, dado que tratemos um sistema com uma grande densidade de fótons $\rho$, o regime perturbativo deixa de ser válido, visto que os elementos da matriz de perturbação são proporcionais a $e^{2} \rho$.

Um problema que podemos resolver exatamente é o caso de um elétron num campo de ondas planas que se propaga numa direção $\boldsymbol{n}$, i.e. ao invés de considerarmos um potencial da forma (2.1.15), usamos um campo $A^{\mu}$ que só contenha fótons que se propagam na direção $\boldsymbol{n}=(0,0,1)$, por exemplo, porém com frequência arbitrária:

$$
|e| A^{\mu}(x)=\sum_{k, \lambda} \sqrt{\frac{\delta}{\omega_{k}}}\left[a_{k, \lambda} e_{\lambda}^{\mu}(k) e^{-i \omega_{k}\left(x^{0}-x^{3}\right)}+a_{k, \lambda}^{\dagger} e_{\lambda}^{* \mu}(k) e^{i \omega_{k}\left(x^{0}-x^{3}\right)}\right]
$$

onde utilizamos $\delta=e^{2} / 2 V \equiv \rho \alpha / 2, \rho=1 / V, \alpha=e^{2}, \boldsymbol{k}=\boldsymbol{n} k$ e $k^{\mu}=\left\{\omega_{k}, \boldsymbol{n} k\right\}$. Como $k^{2}=0$ e $|\boldsymbol{n}|^{2}=1$, então $k=\omega_{k}$. Por simplicidade, usaremos a notação $u^{0}=x^{0}-x^{3}$. 


\subsection{Solução Exata do Problema}

Nesta seção apresentaremos a solução exata da equação de Dirac (2.1.16), tomando $A^{\mu}$ da forma:

$$
|e| A^{\mu}(x)=\sum_{k, \lambda} \sqrt{\frac{\delta}{\omega_{k}}}\left[a_{k, \lambda} e_{\lambda}^{\mu}(k) e^{-i \omega_{k} u_{0}}+a_{k, \lambda}^{\dagger} e_{\lambda}^{* \mu}(k) e^{i \omega_{k} u_{0}}\right], \delta=\frac{e^{2}}{2 V}
$$

i.e. só consideraremos fótons se movendo na direção $\boldsymbol{n}=(0,0,1)$.

\subsubsection{Separação das Variáveis Espaciais e de Fótons}

Considere o operador $G_{\mu}$ de 4-momento total do sistema elétron-fótons:

$$
G_{\mu}=i \partial_{\mu}+n_{\mu} \sum_{k, \lambda} \omega_{k} N_{k, \lambda}
$$

onde

$$
N_{k, \lambda}=a_{k, \lambda}^{\dagger} a_{k, \lambda}, n_{\mu}=(1,-\boldsymbol{n})=(1,0,0,-1) .
$$

Vamos mostrar que $\left[G_{\mu}, P_{\nu}\right]=0, P_{\nu}=i \partial_{\nu}-e A_{\nu}$, e logo $G_{\mu}$ é uma quantidade conservada. Escrevendo explicitamente o comutador, temos:

$$
\begin{aligned}
{\left[i \partial_{\mu}+n_{\mu} \sum_{k} \omega_{k} N_{k, \lambda}, i \partial_{\nu}-e A_{\nu}\right]=} & {\left[i \partial_{\mu}, i \partial_{\nu}\right]+n_{\mu} \sum_{k, \lambda} \omega_{k}\left[N_{k, \lambda}, i \partial_{\nu}\right]-} \\
& -e\left[i \partial_{\mu}, A_{\nu}\right]-e n_{\mu} \sum_{k, \lambda} \omega_{k}\left[N_{k, \lambda}, A_{\nu}\right]
\end{aligned}
$$

É claro que os comutadores $\left[i \partial_{\mu}, i \partial_{\nu}\right]$ e $\left[a_{k, \lambda}^{\dagger} a_{k, \lambda}, i \partial_{\nu}\right]$ são nulos pois os campos tratados são suficientemente bem comportados de forma que as derivadas parciais possam comutar e a parte espacial comuta com a parte de fótons. Nos resta calcular os outros dois comutadores. 
Começamos escrevendo explicitamente o termo $e n_{\mu} \sum_{k, \lambda} \omega_{k}\left[N_{k, \lambda}, A_{\nu}\right]$ :

$$
\begin{aligned}
e n_{\mu} \sum_{k, \lambda} \omega_{k}\left[N_{k, \lambda}, A_{\nu}\right]= & n_{\mu} \sum_{k, \lambda} \sum_{k^{\prime}, \lambda^{\prime}} \frac{e \omega_{k}}{\sqrt{2 \omega_{k^{\prime}} V}}\{\underbrace{\left[N_{k, \lambda}, a_{k^{\prime}, \lambda^{\prime}}\right]}_{=-a_{k, \lambda} \delta_{k k^{\prime}} \delta_{\lambda \lambda^{\prime}}} e_{\lambda^{\prime} \nu}\left(k^{\prime}\right) e^{-i \omega_{k^{\prime}} u_{0}}+ \\
& +\underbrace{\left[N_{k, \lambda}, a_{k^{\prime}, \lambda^{\prime}}^{\dagger}\right]}_{=a_{k, \lambda}^{\dagger} \delta_{k k^{\prime}} \delta_{\lambda^{\prime}}} e_{\lambda^{\prime} \nu}^{*}\left(k^{\prime}\right) e^{i \omega_{k^{\prime}} u_{0}}\} \\
= & -n_{\mu} \sum_{k, \lambda} \frac{e \omega_{k}}{\sqrt{2 \omega_{k} V}}\left[a_{k, \lambda} e_{\lambda \nu}(k) e^{-i \omega_{k} u_{0}}-a_{k, \lambda}^{\dagger} e_{\lambda \nu}^{*}(k) e^{i \omega_{k} u_{0}}\right] \\
e n_{\mu} \sum_{k, \lambda} \omega_{k}\left[N_{k, \lambda}, A_{\nu}\right]= & -e\left[i \partial_{\mu}, A_{\nu}\right]
\end{aligned}
$$

Fica claro então que

$$
\left[G_{\mu}, P_{\nu}\right]=0 .
$$

Como o 4-momento total é uma quantidade conservada, devemos procurar soluções $\Psi(x)$ da equação de Dirac (2.1.16) que sejam autoespinores de $G_{\mu}$, i.e.

$$
G_{\mu} \Psi=g_{\mu} \Psi
$$

onde $g_{\mu}$ são os autovalores de $G_{\mu}$. Consideremos o operador

$$
U_{1}=\exp \left(i \sum_{k, \lambda} k_{\mu} x^{\mu} N_{k, \lambda}\right), k_{\mu}=\omega_{k} n_{\mu} .
$$

Proposição. Se $\Psi=U_{1} \Phi$ e $G_{\mu} \Psi=g_{\mu} \Psi$, então $i \partial_{\mu} \Phi=g_{\mu} \Phi$.

Demonstração. Substituindo $\Psi=U_{1} \Phi$ em $G_{\mu} \Psi=g_{\mu} \Psi$, temos:

$$
G_{\mu} U_{1} \Phi=g_{\mu} U_{1} \Phi \Rightarrow U_{1}^{-1} G_{\mu} U_{1} \Phi=g_{\mu} \Phi
$$

$U_{1}$ é um operador unitário, visto que é da forma $e^{i A}$, onde $A$ é um operador hermitiano, então $U_{1}^{-1}=U_{1}^{\dagger}=\exp \left(-i \sum_{k, \lambda} k_{\mu} x^{\mu} N_{k, \lambda}\right)$. Podemos calcular explicitamente o operador $U_{1}^{-1} G_{\mu} U_{1}$ usando a famosa relação de Hadamard:

$$
e^{X} Y e^{-X}=Y+[X, Y]+\frac{1}{2 !}[X,[X, Y]]+\frac{1}{3 !}[X,[X,[X, Y]]]+\cdots
$$


De forma que

$$
\begin{aligned}
U_{1}^{\dagger} G_{\mu} U_{1} & =\exp \left(-i \sum_{k, \lambda} k_{\nu} x^{\nu} N_{k, \lambda}\right) G_{\mu} \exp \left(i \sum_{k, \lambda} k_{\nu} x^{\nu} N_{k, \lambda}\right) \\
U_{1}^{\dagger} G_{\mu} U_{1} & =G_{\mu}+\left[-i \sum_{k, \lambda} k_{\nu} x^{\nu} N_{k, \lambda}, G_{\mu}\right]+ \\
& +\frac{1}{2 !}\left[-i \sum_{k^{\prime}, \lambda^{\prime}} k_{\nu} x^{\nu} N_{k^{\prime}, \lambda^{\prime}},\left[-i \sum_{k, \lambda} k_{\nu} x^{\nu} N_{k, \lambda}, G_{\mu}\right]\right]+\cdots
\end{aligned}
$$

Calculando o termo $-i \sum_{k, \lambda}\left[k_{\nu} x^{\nu} a_{k, \lambda}^{\dagger} a_{k, \lambda}, G_{\mu}\right]$, temos

$$
\begin{aligned}
-i \sum_{k, \lambda}\left[k_{\nu} x^{\nu} a_{k, \lambda}^{\dagger} a_{k, \lambda}, G_{\mu}\right]= & -i \sum_{k, \lambda}\left[k_{\nu} x^{\nu} N_{k, \lambda}, i \partial_{\mu}+n_{\mu} \sum_{k^{\prime}, \lambda^{\prime}} \omega_{k^{\prime}} N_{k^{\prime}, \lambda^{\prime}}\right] \\
= & i \sum_{k, \lambda}\left[i \partial_{\mu}, k_{\nu} x^{\nu} N_{k, \lambda}\right]+ \\
& +i \sum_{k, \lambda} \sum_{k^{\prime}, \lambda^{\prime}} n_{\mu} \omega_{k^{\prime}} k_{\nu} x^{\nu}\left[N_{k^{\prime}, \lambda^{\prime}}, N_{k, \lambda}\right] \\
-i \sum_{k, \lambda}\left[k_{\nu} x^{\nu} a_{k, \lambda}^{\dagger} a_{k, \lambda}, G_{\mu}\right]= & -n_{\mu} \sum_{k, \lambda} \omega_{k} N_{k, \lambda} .
\end{aligned}
$$

Donde facilmente concluímos que

$$
\begin{aligned}
{\left[-i \sum_{k^{\prime}, \lambda^{\prime}} k_{\nu} x^{\nu} N_{k^{\prime}, \lambda^{\prime}},\left[-i \sum_{k, \lambda} k_{\nu} x^{\nu} N_{k, \lambda}, G_{\mu}\right]\right] } & =i n_{\mu} \sum_{k^{\prime}, \lambda^{\prime}} \sum_{k, \lambda}\left[k_{\nu} x^{\nu} N_{k^{\prime}, \lambda^{\prime}}, \omega_{k} N_{k, \lambda}\right] \\
& =0 .
\end{aligned}
$$

Com isso, todos os comutadores seguintes são nulos e obtemos que

$$
U_{1}^{\dagger} G_{\mu} U_{1}=G_{\mu}-n_{\mu} \sum_{k, \lambda} \omega_{k} N_{k, \lambda}=i \partial_{\mu} .
$$

Isso nos leva a concluir que, de fato

$$
i \partial_{\mu} \Phi=g_{\mu} \Phi
$$


A equação (2.2.5) implica que a função $\Phi$ pode ser escrita na forma:

$$
\Phi(x)=e^{-i g_{\mu} x^{\mu}} \tilde{\Phi}
$$

onde $\tilde{\Phi}$ não depende das variáveis espaciais do sistema.

Proposição. Como $\Psi=U_{1} \Phi=U_{1}\left(e^{-i g_{\mu} x^{\mu}} \tilde{\Phi}\right)$ satisfaz a equação de Dirac (2.1.16), então $\tilde{\Phi}$ satisfaz a seguinte equação:

$$
\begin{gathered}
\left(\gamma^{\mu} \tilde{P}_{\mu}-m\right) \tilde{\Phi}=0 \\
\tilde{P}_{\mu}=g_{\mu}-n_{\mu} \sum_{k, \lambda} \omega_{k} N_{k, \lambda}-\epsilon Q_{\mu}, \epsilon=\frac{e}{|e|} \\
Q^{\mu}=\epsilon e U_{1}^{\dagger} A^{\mu} U_{1}=\sum_{k, \lambda} \sqrt{\frac{\delta}{\omega_{k}}}\left[a_{k, \lambda} e_{\lambda}^{\mu}(k)+a_{k, \lambda}^{\dagger} e_{\lambda}^{* \mu}(k)\right], \delta=\frac{e^{2}}{2 V} .
\end{gathered}
$$

Demonstração. Substituindo $\Psi=U_{1} \Phi$ na equação de Dirac (2.1.16), obtemos:

$$
\left(\gamma^{\mu} P_{\mu}-m\right) U_{1} \Phi=0 \Rightarrow\left(\gamma^{\mu} U_{1}^{\dagger} P_{\mu} U_{1}-m\right) \Phi=0
$$

Para calcular $U_{1}^{\dagger} P_{\mu} U_{1}$, usamos a expressão (2.2.3). Como $P_{\mu}=i \partial_{\mu}-e A_{\mu}$, dividiremos o cálculo em duas etapas, primeiramente calcularemos $U_{1}^{\dagger} i \partial_{\mu} U_{1}$ e em seguida $U_{1}^{\dagger} A_{\mu} U_{1}$.

$$
\begin{aligned}
U_{1}^{\dagger} i \partial_{\mu} U_{1} & =U_{1}^{\dagger}\left(G_{\mu}-n_{\mu} \sum_{k, \lambda} \omega_{k} N_{k, \lambda}\right) U_{1} \\
& =U_{1}^{\dagger} G_{\mu} U_{1}-n_{\mu} \sum_{k, \lambda} \omega_{k} U_{1}^{\dagger} N_{k, \lambda} U_{1} \\
U_{1}^{\dagger} i \partial_{\mu} U_{1} & =i \partial_{\mu}-n_{\mu} \sum_{k, \lambda} \omega_{k} N_{k, \lambda},
\end{aligned}
$$

onde usamos a equação (2.2.4). Caculemos agora $U_{1}^{\dagger} A_{\mu} U_{1}$ :

$$
U_{1}^{\dagger} A_{\mu} U_{1}=\sum_{k, \lambda} \frac{1}{\sqrt{2 \omega_{k} V}}\left[U_{1}^{\dagger} a_{k, \lambda} U_{1} e_{\lambda}^{\mu}(k) e^{-i \omega_{k} u_{0}}+U_{1}^{\dagger} a_{k, \lambda}^{\dagger} U_{1} e_{\lambda}^{* \mu}(k) e^{i \omega_{k} u_{0}}\right]
$$


O termo que devemos explicitar é $U_{1}^{\dagger} a_{k, \lambda} U_{1}$ :

$$
\begin{aligned}
U_{1}^{\dagger} a_{k, \lambda} U_{1}= & a_{k, \lambda}+\sum_{k^{\prime}, \lambda^{\prime}}\left(-i k_{\mu} x^{\mu}\right) \underbrace{\left[N_{k^{\prime}, \lambda^{\prime}}, a_{k, \lambda}\right]}_{=-a_{k, \lambda} \delta_{k^{\prime} k} \delta_{\lambda^{\prime} \lambda}}+ \\
& +\frac{1}{2 !} \sum_{k^{\prime}, \lambda^{\prime}} \sum_{k^{\prime \prime}, \lambda^{\prime \prime}}\left(-i k_{\mu} x^{\mu}\right)^{2}\left[N_{k^{\prime \prime}, \lambda^{\prime \prime}},\left[N_{k^{\prime}, \lambda^{\prime}}, a_{k, \lambda}\right]\right]+\cdots \\
= & a_{k, \lambda}\left[1+i k_{\mu} x^{\mu}+\frac{1}{2 !}\left(i k_{\mu} x^{\mu}\right)^{2}+\cdots\right] \\
U_{1}^{\dagger} a_{k, \lambda} U_{1}= & a_{k, \lambda} e^{i k_{\mu} x^{\mu}}=a_{k, \lambda} e^{i \omega_{k} u_{0}}
\end{aligned}
$$

O termo $U_{1}^{\dagger} a_{k, \lambda}^{\dagger} U_{1}$ é apenas o adjunto hermitiano de $U_{1}^{\dagger} a_{k, \lambda} U_{1}$, de tal forma que obtemos:

$$
\begin{aligned}
e U_{1}^{\dagger} A_{\mu} U_{1} & =\sum_{k, \lambda} \frac{e}{|e|} \sqrt{\frac{e^{2}}{2 \omega_{k} V}}\left[a_{k, \lambda} e_{\lambda}^{\mu}(k)+a_{k, \lambda}^{\dagger} e_{\lambda}^{* \mu}(k)\right] \\
& =\epsilon \sum_{k, \lambda} \sqrt{\frac{\delta}{\omega_{k}}}\left[a_{k, \lambda} e_{\lambda}^{\mu}(k)+a_{k, \lambda}^{\dagger} e_{\lambda}^{* \mu}(k)\right] \\
e U_{1}^{\dagger} A_{\mu} U_{1} & =\epsilon Q_{\mu} .
\end{aligned}
$$

Concluímos que

$$
U_{1}^{\dagger} P_{\mu} U_{1}=i \partial_{\mu}-n_{\mu} \sum_{k, \lambda} \omega_{k} N_{k, \lambda}-\epsilon Q_{\mu} .
$$

Podemos prontamente obter qual equação $\tilde{\Phi}$ satisfaz:

$$
\left(\gamma^{\mu} U_{1}^{\dagger} P_{\mu} U_{1}-m\right)\left(e^{-i g_{\mu} x^{\mu}} \tilde{\Phi}\right)=0
$$

que nos dá:

$$
\left[\gamma^{\mu}\left(i \partial_{\mu}-n_{\mu} \sum_{k, \lambda} \omega_{k} N_{k, \lambda}-\epsilon Q_{\mu}\right)-m\right]\left(e^{-i g_{\mu} x^{\mu}} \tilde{\Phi}\right)=0
$$

e portanto

$$
\left(\gamma^{\mu} \tilde{P}_{\mu}-m\right) \tilde{\Phi}=0
$$

onde

$$
\tilde{P}_{\mu}=g_{\mu}-n_{\mu} \sum_{k, \lambda} \omega_{k} N_{k, \lambda}-\epsilon Q_{\mu}
$$

A equação (2.2.6) é um resultado notável, visto que essa equação só envolve variáveis dos fótons e não mais variáveis espaciais. 


\subsubsection{Separação de Variáveis de Spin na Equação Fotônica}

Como vimos, $\tilde{\Phi}$ é um espinor de quatro componentes que descreve completamente a parte fotônica do sistema e satisfaz a equação

$$
\left[\not p-\not h \sum_{k, \lambda} \omega_{k} N_{k, \lambda}-\epsilon \emptyset-m\right] \tilde{\Phi}=0
$$

onde estamos usando a notação de Feynman, i.e. $\not g \equiv \gamma_{\mu} g^{\mu}$, por exemplo. No que segue, mostraremos que o operador

$$
U_{2}=1+\epsilon \frac{\not \phi}{2(n \cdot g)}
$$

realiza a separação de variáveis de spin em (2.2.9) se escrevermos $\tilde{\Phi}=U_{2} \Xi$ e fizermos a escolha $\Xi=F u$, com $F$ sendo uma função e $u$ um espinor de Dirac constante.

Proposição. $O$ inverso do operador $U_{2}$ é dado por:

$$
U_{2}^{-1}=1-\epsilon \frac{\not \phi Q}{2(n \cdot g)} .
$$

Demonstração. Para verificarmos esse fato devemos mostrar que $U_{2} U_{2}^{-1}=1$. Efetuando o cálculo $U_{2} U_{2}^{-1}$ obtemos:

$$
\begin{aligned}
U_{2} U_{2}^{-1} & =\left(1+\epsilon \frac{\not \phi}{2(n \cdot g)}\right)\left(1-\epsilon \frac{\not \phi}{2(n \cdot g)}\right) \\
& =1-\frac{\epsilon^{2}}{4(n \cdot g)^{2}} \not \phi \not \phi .
\end{aligned}
$$

Explicitando o termo $h \varnothing \not \phi:$

$$
\begin{aligned}
\not h \phi \not \phi & =\gamma_{\alpha} \gamma_{\beta} \gamma_{\delta} \gamma_{\sigma} n^{\alpha} n^{\delta} Q^{\beta} Q^{\sigma} \\
& =\gamma_{\alpha}\left(-\gamma_{\delta} \gamma_{\beta}+2 \eta_{\beta \delta}\right) \gamma_{\sigma} n^{\alpha} n^{\delta} Q^{\beta} Q^{\sigma} \\
\not \phi \phi \not \phi & =-\gamma_{\alpha} \gamma_{\delta} \gamma_{\beta} \gamma_{\sigma} n^{\alpha} n^{\delta} Q^{\beta} Q^{\sigma}+2 \gamma_{\alpha} \gamma_{\sigma} \underbrace{(n \cdot Q)}_{=0} .
\end{aligned}
$$

Note que o termo $\gamma_{\alpha} \gamma_{\delta} n^{\alpha} n^{\delta}$ é nulo pois:

$$
\begin{aligned}
\gamma_{\alpha} \gamma_{\delta} n^{\alpha} n^{\delta} & =\left(-\gamma_{\delta} \gamma_{\alpha}+2 \eta_{\alpha \delta}\right) n^{\alpha} n^{\delta} \\
& =-\gamma_{\delta} \gamma_{\alpha} n^{\alpha} n^{\delta}+2 n^{2} \\
\gamma_{\alpha} \gamma_{\delta} n^{\alpha} n^{\delta} & =-\gamma_{\alpha} \gamma_{\delta} n^{\delta} n^{\alpha}+2 n^{2} .
\end{aligned}
$$


Essa última linha nos permite concluir que

$$
\gamma_{\alpha} \gamma_{\delta} n^{\alpha} n^{\delta}=n^{2}=0
$$

o que nos diz que $\not h \not h \phi=0$ e isso completa nossa verificação.

Procuremos a equação que $\Xi$ satisfaz. Substituindo $\tilde{\Phi}=U_{2} \Xi$ na equação (2.2.9) e multiplicado a mesma por $U_{2}^{-1}$ pela esquerda, obtemos:

$$
[\underbrace{U_{2}^{-1} \not g U_{2}}_{(1)}-\sum_{k, \lambda} \omega_{k} \underbrace{U_{2}^{-1} \not h N_{k, \lambda} U_{2}}_{(2)}-\underbrace{\epsilon U_{2}^{-1} \phi U_{2}}_{(3)}-m] \Xi=0 .
$$

Vamos calcular os termos dessa equação separadamente.

1. $U_{2}^{-1} \not g U_{2}$ :

$$
\begin{aligned}
U_{2}^{-1} \not g U_{2} & =\left(1-\epsilon \frac{\not \phi}{2(n \cdot g)}\right) \not g\left(1+\epsilon \frac{\hbar \phi}{2(n \cdot g)}\right) \\
& =\not \phi+\frac{\epsilon}{2(n \cdot g)}[\phi, \not \phi]-\frac{\epsilon^{2}}{4(n \cdot g)^{2}} \not \phi \phi \phi \phi
\end{aligned}
$$

Escrevendo explicitamente os termos $[\not, \not h \phi]$ e $\not \phi \phi, \phi h \phi$, obtemos:

$$
\begin{gathered}
{[\not \phi, \not h \phi]=g^{\mu} n^{\nu} Q^{\sigma}\left[\gamma_{\mu}, \gamma_{\nu} \gamma_{\sigma}\right]} \\
=g^{\mu} n^{\nu} Q^{\sigma}\left(\left\{\gamma_{\mu}, \gamma_{\nu}\right\} \gamma_{\sigma}-\gamma_{\nu}\left\{\gamma_{\mu}, \gamma_{\sigma}\right\}\right) \\
=2 g^{\mu} n^{\nu} Q^{\sigma}\left(\eta_{\mu \nu} \gamma_{\sigma}-\gamma_{\nu} \eta_{\mu \sigma}\right)
\end{gathered}
$$




$$
\therefore \not \phi \phi \phi \phi Q=2(n \cdot g) \not h \gamma_{\nu} \gamma_{\beta} Q^{\nu} Q^{\beta}
$$

Olhando mais atentamente para o termo $\gamma_{\nu} \gamma_{\beta} Q^{\nu} Q^{\beta}$ :

$$
\begin{aligned}
\gamma_{\nu} \gamma_{\beta} Q^{\nu} Q^{\beta} & =\left(-\gamma_{\beta} \gamma_{\nu}+2 \eta_{\nu \beta}\right) Q^{\nu} Q^{\beta} \\
& =-\gamma_{\beta} \gamma_{\nu} Q^{\nu} Q^{\beta}+2 Q^{2} \\
& =-\gamma_{\nu} \gamma_{\beta} Q^{\beta} Q^{\nu}+2 Q^{2} \\
\gamma_{\nu} \gamma_{\beta} Q^{\nu} Q^{\beta} & =-\gamma_{\nu} \gamma_{\beta} Q^{\nu} Q^{\beta}-\gamma_{\nu} \gamma_{\beta}\left[Q^{\beta}, Q^{\nu}\right]+2 Q^{2}
\end{aligned}
$$

O que nos diz que

$$
\gamma_{\nu} \gamma_{\beta} Q^{\nu} Q^{\beta}=Q^{2}+\frac{1}{2} \gamma_{\nu} \gamma_{\beta}\left[Q^{\nu}, Q^{\beta}\right] .
$$

Explicitando o termo $\left[Q^{\nu}, Q^{\beta}\right]$ :

$$
\begin{aligned}
{\left[Q^{\nu}, Q^{\beta}\right] } & =\sum_{k, \lambda} \sum_{k^{\prime}, \lambda^{\prime}} \sqrt{\frac{\delta}{\omega_{k}}} \sqrt{\frac{\delta}{\omega_{k^{\prime}}}}\left\{\left[a_{k, \lambda}, a_{k^{\prime}, \lambda^{\prime}}^{\dagger}\right] e_{\lambda}^{\nu} e_{\lambda^{\prime}}^{* \beta}+\left[a_{k, \lambda}^{\dagger}, a_{k^{\prime}, \lambda^{\prime}}\right] e_{\lambda^{\prime}}^{\beta} e_{\lambda}^{* \nu}\right\} \\
& =\sum_{k, \lambda} \frac{\delta}{\omega_{k}}\left(e_{\lambda}^{\nu} e_{\lambda}^{* \beta}-e_{\lambda}^{\beta} e_{\lambda}^{* \nu}\right)=\sum_{k, \lambda} \frac{\delta}{\omega_{k}}\left(\delta_{\nu^{\prime}}^{\nu} \delta_{\beta^{\prime}}^{\beta}-\delta_{\nu^{\prime}}^{\beta} \delta_{\beta^{\prime}}^{\nu}\right) e_{\lambda}^{\nu^{\prime}} e_{\lambda}^{* \beta^{\prime}} \\
& =\frac{1}{2} \sum_{k, \lambda} \frac{\delta}{\omega_{k}} \epsilon_{\delta \sigma \nu^{\prime} \beta^{\prime}} \epsilon^{\delta \sigma \nu \beta} e_{\lambda}^{\nu^{\prime}} e_{\lambda}^{* \beta^{\prime}} \\
& =\frac{1}{2} \sum_{k, \lambda} \frac{\delta}{\omega_{k}}\left[\epsilon^{0 \sigma \nu \beta} \epsilon_{0 \sigma \nu^{\prime} \beta^{\prime}} e_{\lambda}^{\nu^{\prime}} e_{\lambda}^{* \beta^{\prime}}+\epsilon^{\delta 0 \nu \beta} \epsilon_{\delta 0 \nu^{\prime} \beta^{\prime}} e_{\lambda}^{\nu^{\prime}} e_{\lambda}^{* \beta^{\prime}}\right] \\
{\left[Q^{\nu}, Q^{\beta}\right] } & =\sum_{k, \lambda} \frac{\delta}{\omega_{k}} \epsilon^{0 \sigma \nu \beta}\left(\boldsymbol{e}_{\lambda} \times \boldsymbol{e}_{\lambda^{\prime}}^{*}\right)_{\sigma},
\end{aligned}
$$

de forma que

$$
\gamma_{\nu} \gamma_{\beta}\left[Q^{\nu}, Q^{\beta}\right]=\sum_{k, \lambda} \frac{\delta}{\omega_{k}} \gamma_{\nu} \gamma_{\beta} \epsilon^{0 \sigma \nu \beta}\left(\boldsymbol{e}_{\lambda} \times \boldsymbol{e}_{\lambda^{\prime}}^{*}\right)_{\sigma}
$$


Como $\boldsymbol{e}_{\lambda} \times \boldsymbol{e}_{\lambda^{\prime}}^{*} \propto \boldsymbol{n}$, então, no caso de $\boldsymbol{n}=(0,0,1)$, obtemos que:

$$
\begin{aligned}
\gamma_{\nu} \gamma_{\beta}\left[Q^{\nu}, Q^{\beta}\right] & =\sum_{k, \lambda} \frac{\delta}{\omega_{k}} \gamma_{\nu} \gamma_{\beta} \epsilon^{03 \nu \beta}\left[\boldsymbol{n} \cdot\left(\boldsymbol{e}_{\lambda} \times \boldsymbol{e}_{\lambda^{\prime}}^{*}\right)\right] \\
& =\gamma_{0} \gamma_{3} \sum_{k, \lambda} \frac{\delta}{\omega_{k}} \gamma_{0} \gamma_{3} \gamma_{\nu} \gamma_{\beta} \epsilon^{03 \nu \beta}\left[\boldsymbol{n} \cdot\left(\boldsymbol{e}_{\lambda} \times \boldsymbol{e}_{\lambda^{\prime}}^{*}\right)\right] \\
& =\gamma_{0} \gamma_{3} \sum_{k, \lambda} \frac{\delta}{\omega_{k}}\left(\gamma_{0} \gamma_{3} \gamma_{1} \gamma_{2}-\gamma_{0} \gamma_{3} \gamma_{2} \gamma_{1}\right)\left[\boldsymbol{n} \cdot\left(\boldsymbol{e}_{\lambda} \times \boldsymbol{e}_{\lambda^{\prime}}^{*}\right)\right] \\
& =-2 i \gamma_{0} \gamma_{3} \sum_{k, \lambda} \frac{\delta}{\omega_{k}} \gamma_{5}\left[\boldsymbol{n} \cdot\left(\boldsymbol{e}_{\lambda} \times \boldsymbol{e}_{\lambda^{\prime}}^{*}\right)\right] \\
\gamma_{\nu} \gamma_{\beta}\left[Q^{\nu}, Q^{\beta}\right] & =-4(n \cdot g) a \gamma_{5} \gamma_{0} \gamma_{3}
\end{aligned}
$$

onde

$$
a=i \sum_{k, \lambda} \frac{\delta}{2(n \cdot g) \omega_{k}}\left[\boldsymbol{n} \cdot\left(\boldsymbol{e}_{\lambda} \times \boldsymbol{e}_{\lambda^{\prime}}^{*}\right)\right]
$$

e portanto

$$
\gamma_{\nu} \gamma_{\beta} Q^{\nu} Q^{\beta}=Q^{2}-2(n \cdot g) a \gamma_{5} \gamma_{0} \gamma_{3}
$$

Finalmente, obtemos que

$$
\not \phi \phi \not h \phi=2(n \cdot g) \not h\left(Q^{2}-2(n \cdot g) a \gamma_{5} \gamma_{0} \gamma_{3}\right),
$$

o que nos leva a concluir que

$$
U_{2}^{-1} \not g U_{2}=\not g+\epsilon \not Q-\not h\left[\epsilon \frac{(g \cdot Q)}{(n \cdot g)}+\frac{Q^{2}}{2(n \cdot g)}-a \gamma_{5} \gamma_{0} \gamma_{3}\right] \text {. }
$$

2. $U_{2}^{-1} \not h N_{k, \lambda} U_{2}$ :

$$
\begin{aligned}
U_{2}^{-1} \not h N_{k, \lambda} U_{2} & =\left(1-\epsilon \frac{\not \phi}{2(n \cdot g)}\right) \not h N_{k, \lambda}\left(1+\epsilon \frac{\not \phi \phi}{2(n \cdot g)}\right) \\
& =\not h N_{k, \lambda}+\frac{\epsilon}{2(n \cdot g)}\left[\not h N_{k, \lambda}, \not h \phi\right]-\frac{\epsilon^{2}}{4(n \cdot g)^{2}} \underbrace{\not h \phi h N_{k, \lambda} \not \phi \phi}_{=0}
\end{aligned}
$$


Escrevendo explicitamente o termo $\left[\not h N_{k, \lambda}, \not h \phi\right]$, obtemos:

$$
\begin{aligned}
{\left[\not h N_{k, \lambda}, \not h \phi\right] } & =n^{\mu} n^{\nu}\left[\gamma_{\mu} N_{k, \lambda}, \gamma_{\nu} \gamma_{\sigma} Q^{\sigma}\right] \\
& =n^{\mu} n^{\nu}\left\{\gamma_{\mu}\left[N_{k, \lambda}, \gamma_{\nu} \gamma_{\sigma} Q^{\sigma}\right]+\left[\gamma_{\mu}, \gamma_{\nu} \gamma_{\sigma} Q^{\sigma}\right] N_{k, \lambda}\right\} \\
& =n^{\mu} n^{\nu}\left\{\gamma_{\mu} \gamma_{\nu} \gamma_{\sigma}\left[N_{k, \lambda}, Q^{\sigma}\right]+\left[\gamma_{\mu}, \gamma_{\nu} \gamma_{\sigma}\right] Q^{\sigma} N_{k, \lambda}\right\} \\
& =n^{\mu} n^{\nu}\left\{\gamma_{\mu} \gamma_{\nu} \gamma_{\sigma}\left[N_{k, \lambda}, Q^{\sigma}\right]+2\left(\eta_{\mu \nu} \gamma_{\sigma}-\gamma_{\nu} \eta_{\mu \sigma}\right) Q^{\sigma} N_{k, \lambda}\right\} \\
{\left[\not h N_{k, \lambda}, \not h \phi\right] } & =0 .
\end{aligned}
$$

Portanto

$$
U_{2}^{-1} \not h N_{k, \lambda} U_{2}=\not h N_{k, \lambda} \text {. }
$$

3. $\epsilon U_{2}^{-1} \phi U_{2}$ :

$$
\begin{aligned}
\epsilon U_{2}^{-1} \phi U_{2} & =\left(1-\epsilon \frac{\not \phi}{2(n \cdot g)}\right) \epsilon \phi\left(1+\epsilon \frac{\not h \phi}{2(n \cdot g)}\right) \\
& =\epsilon \phi+\frac{\epsilon^{2}}{2(n \cdot g)}[\phi, \not \phi]-\frac{\epsilon^{3}}{4(n \cdot g)} \not \phi \phi \hbar \phi
\end{aligned}
$$

Escrevendo explicitamente os termos $[\phi, \not \phi]$ e $\not \pitchfork \phi \phi \not \phi, ~ o b t e m o s:$

$$
\begin{aligned}
{[\phi, \not \phi \phi] } & =n^{\sigma}\left[\gamma_{\mu} Q^{\mu}, \gamma_{\sigma} \gamma_{\nu} Q^{\nu}\right] \\
& =n^{\sigma}\left\{\gamma_{\mu} \gamma_{\sigma} \gamma_{\nu}\left[Q^{\mu}, Q^{\nu}\right]+\left[\gamma_{\mu}, \gamma_{\sigma} \gamma_{\nu}\right] Q^{\nu} Q^{\mu}\right\} \\
& =n^{\sigma}\left\{\gamma_{\mu} \gamma_{\sigma} \gamma_{\nu}+2\left(\eta_{\mu \sigma} \gamma_{\nu}-\gamma_{\sigma} \eta_{\mu \nu}\right) Q^{\nu} Q^{\mu}\right\} \\
& =n^{\sigma}\left\{\left(-\gamma_{\sigma} \gamma_{\mu}+2 \eta_{\sigma \mu}\right) \gamma_{\nu}\left[Q^{\mu}, Q^{\nu}\right]-2 \gamma_{\sigma} Q^{2}\right\} \\
& =-\not h\left(\gamma_{\mu} \gamma_{\nu}\left[Q^{\mu}, Q^{\nu}\right]+2 Q^{2}\right) \\
{[\phi, \not \phi Q] } & =-\not h\left\{2 Q^{2}-4(n \cdot g) a \gamma_{5} \gamma_{0} \gamma_{3}\right\}
\end{aligned}
$$




$$
\begin{aligned}
\not \phi \phi \hbar \phi & =\not\left(Q^{2}+\frac{1}{2} \gamma_{\mu} \gamma_{\nu}\left[Q^{\mu}, Q^{\nu}\right]\right) \not \phi \\
& =\underbrace{\not Q^{2} \not \phi}_{=0}+\frac{1}{2} \not h \gamma_{\mu} \gamma_{\nu}\left[Q^{\mu}, Q^{\nu}\right] \not \phi \\
& =\frac{1}{2} n^{\alpha} n^{\beta} \gamma_{\alpha} \gamma_{\mu} \gamma_{\nu} \gamma_{\beta}\left[Q^{\mu}, Q^{\nu}\right] \gamma_{\delta} Q^{\delta} \\
& =\frac{1}{2} n^{\alpha} n^{\beta} \gamma_{\alpha} \gamma_{\mu}\left(-\gamma_{\beta} \gamma_{\nu}+2 \eta_{\nu \beta}\right)\left[Q^{\mu}, Q^{\nu}\right] \gamma_{\delta} Q^{\delta} \\
& =-\frac{1}{2} n^{\alpha} n^{\beta} \gamma_{\alpha}\left(-\gamma_{\beta} \gamma_{\mu}+2 \eta_{\mu \beta}\right) \gamma_{\nu}\left[Q^{\mu}, Q^{\nu}\right] \gamma_{\delta} Q^{\delta} \\
& =\frac{1}{2} \underbrace{n^{\alpha} n^{\beta} \gamma_{\alpha} \gamma_{\beta}}_{=0} \gamma_{\mu} \gamma_{\nu}\left[Q^{\mu}, Q^{\nu}\right] \gamma_{\delta} Q^{\delta} \\
\not \phi \phi \hbar \phi & =0 .
\end{aligned}
$$

Esses resultados nos dizem que

$$
\epsilon U_{2}^{-1} \not U_{2}=\epsilon \not 2-\frac{1}{2(n \cdot g)} \not h\left\{2 Q^{2}-4(n \cdot g) a \gamma_{5} \gamma_{0} \gamma_{3}\right\}
$$

e, portanto,

$$
\epsilon U_{2}^{-1} \phi U_{2}=\epsilon \mathscr{L}-\not h\left\{\frac{Q^{2}}{(n \cdot g)}-2 a \gamma_{5} \gamma_{0} \gamma_{3}\right\}
$$

Reunindo os resultados (2.2.10), (2.2.11) e (2.2.12), chegamos em:

$$
\left[\not p-\not h\left(\sum_{k, \lambda} \omega_{k} N_{k, \lambda}+\epsilon \frac{(g \cdot Q)}{(n \cdot g)}-\frac{Q^{2}}{2(n \cdot g)}+a \gamma_{5} \gamma_{0} \gamma_{3}\right)-m\right] \Xi=0
$$

Proposição. A equação de Klein-Gordon

$$
\left(\tilde{P}_{\mu} \tilde{P}^{\mu}-m^{2}\right) F=0
$$

pode ser rescrita como

$$
\left(\sum_{k, \lambda} \omega_{k} N_{k, \lambda}+\epsilon \frac{(g \cdot Q)}{(n \cdot g)}-\frac{Q^{2}}{2(n \cdot g)}\right) F=R F
$$

se $g_{\mu}=p_{\mu}+R n_{\mu}$, onde $R$ é uma constante. 
Demonstração. Escrevendo explicitamente o termo $\tilde{P}_{\mu} \tilde{P}^{\mu}$ :

$$
\begin{aligned}
\tilde{P}_{\mu} \tilde{P}^{\mu} & =\left(g_{\mu}-n_{\mu} \sum_{k, \lambda} \omega_{k} N_{k, \lambda}-\epsilon Q_{\mu}\right)\left(g^{\mu}-n^{\mu} \sum_{k^{\prime}, \lambda^{\prime}} \omega_{k^{\prime}} N_{k^{\prime}, \lambda^{\prime}}-\epsilon Q^{\mu}\right) \\
& =g^{2}-2(n \cdot g) \sum_{k, \lambda} \omega_{k} N_{k, \lambda}-2 \epsilon(g \cdot Q)+Q^{2}
\end{aligned}
$$

Como $g_{\mu}=p_{\mu}+R n_{\mu}$, então $g^{2}=p^{2}+2 R(n \cdot p)$. Adicionando os fatos de que $p^{2}=m^{2} \mathrm{e}$ $(n \cdot p)=(n \cdot g)$, concluímos que $g^{2}=m^{2}+2 R(n \cdot g)$. Dessa forma,

$$
\begin{gathered}
\left(\tilde{P}_{\mu} \tilde{P}^{\mu}-m^{2}\right) F=0 \Rightarrow \\
\Rightarrow\left[m^{2}+2 R(n \cdot g)-2(n \cdot g) \sum_{k, \lambda} \omega_{k} N_{k, \lambda}-2 \epsilon(g \cdot Q)+Q^{2}-m^{2}\right] F=0,
\end{gathered}
$$

o que nos leva ao resultado

$$
\left(\sum_{k, \lambda} \omega_{k} N_{k, \lambda}+\epsilon \frac{(g \cdot Q)}{(n \cdot g)}-\frac{Q^{2}}{2(n \cdot g)}\right) F=R F
$$

Supondo $\Xi=F u$, onde $F$ é uma função escalar que satisfaz (2.2.14), com autovalor $R$, e $u$ é um espinor constante que é determinado pela solução da equação:

$$
\left[\not p-\not h\left(R+a \gamma_{5} \gamma_{0} \gamma_{3}\right)-m\right] u=0 \text {. }
$$

É conveniente escolher $u$ na base de autovetores de $\gamma_{0} \gamma_{3}$, i.e.

$$
\gamma_{0} \gamma_{3} u=s u, s= \pm 1
$$

Dessa forma, a equação que devemos resolver é:

$$
\left[\not p-\not h\left(R+s a \gamma_{5}\right)-m\right] u=0 .
$$

Essa equação só admite solução não trivial no caso em que

$$
\operatorname{det}\left[\not g-\not h\left(R+s a \gamma_{5}\right)-m\right]=0 \text {. }
$$


O determinante de $\left[\not p-\not h\left(R+s a \gamma_{5}\right)-m\right]$ é dado por:

$$
\operatorname{det}\left[\not h-\not h\left(R+s a \gamma_{5}\right)-m\right]=\left[(g-n R-n s a)^{2}-m^{2}\right]\left[(g-n R+n s a)^{2}-m^{2}\right],
$$

de forma que é nulo nos casos $g_{\mu}=p_{\mu}+n_{\mu}(R+s a)$ ou $g_{\mu}=p_{\mu}+n_{\mu}(R-s a)$.

\subsubsection{Solução da Equação de Klein-Gordon}

Como foi expressado na proposição 2.2.2, as soluções da equação de Klein-Gordon (2.2.14) têm um papel importante na solução da equação de Dirac (2.2.6). Nessa seção vamos nos concentrar em encontrar as soluções de (2.2.14). O método que usaremos para encontrar as soluções dessa equação consiste em reduzi lá a uma forma canônica.

\subsubsection{Redução à Forma Normal}

Começamos escrevendo explicitamente os termos $\epsilon(g \cdot Q) /(n \cdot g)$ e $Q^{2} / 2(n \cdot g)$ de $(2.2 .14)$ :

$$
\begin{aligned}
\epsilon \frac{(g \cdot Q)}{(n \cdot g)} & =\sum_{k, \lambda} \frac{\epsilon}{(n \cdot p)} \sqrt{\frac{\delta}{\omega_{k}}}\left[a_{k, \lambda}\left(p \cdot e_{\lambda}\right)+a_{k, \lambda}^{\dagger}\left(p \cdot e_{\lambda}^{*}\right)\right] \\
& =-\sum_{k, \lambda}\left[\tilde{D}_{k, \lambda} a_{k, \lambda}+\text { c.c. }\right]
\end{aligned}
$$

onde

$$
\tilde{D}_{k, \lambda} \equiv \epsilon \sqrt{\frac{\delta}{\omega_{k}}} \frac{\left(\boldsymbol{p} \cdot \boldsymbol{e}_{\lambda}\right)}{(n \cdot p)} .
$$


Agora trataremos do termo $Q^{2} / 2(n \cdot g)$ :

$$
\begin{aligned}
& \frac{Q^{2}}{2(n \cdot g)}=\sum_{\substack{k, k^{\prime} \\
\lambda, \lambda^{\prime}}} \frac{\delta}{2(n \cdot p) \sqrt{\omega_{k} \omega_{k^{\prime}}}}\left[a_{k, \lambda} e_{\lambda}^{\mu}+a_{k, \lambda}^{\dagger} e_{\lambda}^{* \mu}\right]\left[a_{k^{\prime}, \lambda^{\prime}} e_{\lambda^{\prime} \mu}+a_{k^{\prime}, \lambda^{\prime}}^{\dagger} e_{\lambda^{\prime} \mu}^{*}\right] \\
& =\sum_{\substack{k, k^{\prime} \\
\lambda, \lambda^{\prime}}} \frac{\delta}{2(n \cdot p) \sqrt{\omega_{k} \omega_{k^{\prime}}}}\left[a_{k, \lambda} a_{k^{\prime}, \lambda^{\prime}}\left(e_{\lambda}^{\mu} e_{\lambda^{\prime} \mu}\right)+a_{k, \lambda} a_{k^{\prime}, \lambda^{\prime}}^{\dagger}\left(e_{\lambda}^{\mu} e_{\lambda^{\prime} \mu}^{*}\right)+\right. \\
& \left.+a_{k, \lambda}^{\dagger} a_{k^{\prime}, \lambda^{\prime}}\left(e_{\lambda}^{* \mu} e_{\lambda^{\prime} \mu}\right)+a_{k, \lambda}^{\dagger} a_{k^{\prime}, \lambda^{\prime}}^{\dagger}\left(e_{\lambda}^{* \mu} e_{\lambda^{\prime} \mu}^{*}\right)\right] \\
& =\sum_{\substack{k, k^{\prime} \\
\lambda, \lambda^{\prime}}} \frac{\delta}{2(n \cdot p) \sqrt{\omega_{k} \omega_{k^{\prime}}}}\left[a_{k, \lambda} a_{k^{\prime}, \lambda^{\prime}}\left(e_{\lambda}^{\mu} e_{\lambda^{\prime} \mu}\right)+\left(a_{k^{\prime}, \lambda^{\prime}}^{\dagger} a_{k, \lambda}+\delta_{k k^{\prime}} \delta_{\lambda \lambda^{\prime}}\right)\left(e_{\lambda}^{\mu} e_{\lambda^{\prime} \mu}^{*}\right)+\right. \\
& \left.+a_{k, \lambda}^{\dagger} a_{k^{\prime}, \lambda^{\prime}}\left(e_{\lambda}^{* \mu} e_{\lambda^{\prime} \mu}\right)+a_{k, \lambda}^{\dagger} a_{k^{\prime}, \lambda^{\prime}}^{\dagger}\left(e_{\lambda}^{* \mu} e_{\lambda^{\prime} \mu}^{*}\right)\right] \\
& \frac{Q^{2}}{2(n \cdot g)}=\sum_{\substack{k, k^{\prime} \\
\lambda, \lambda^{\prime}}} \frac{\delta}{2(n \cdot p) \sqrt{\omega_{k} \omega_{k^{\prime}}}}\left[2 a_{k, \lambda}^{\dagger} a_{k^{\prime}, \lambda^{\prime}}\left(e_{\lambda}^{* \mu} e_{\lambda^{\prime} \mu}\right)+a_{k, \lambda} a_{k^{\prime}, \lambda^{\prime}}\left(e_{\lambda}^{\mu} e_{\lambda^{\prime} \mu}\right)+\right. \\
& \left.+a_{k, \lambda}^{\dagger} a_{k^{\prime}, \lambda^{\prime}}^{\dagger}\left(e_{\lambda}^{* \mu} e_{\lambda^{\prime} \mu}^{*}\right)\right]+\sum_{k, \lambda} \frac{\delta}{2(n \cdot p) \omega_{k}} \underbrace{\left(e_{\lambda}^{\mu} e_{\lambda \mu}^{*}\right)}_{=-1}
\end{aligned}
$$

Juntando esses resultados na (2.2.14), obtemos:

$$
\begin{gathered}
{\left[\sum_{\substack{k, k^{\prime} \\
\lambda, \lambda^{\prime}}}\left(\tilde{A}_{k \lambda, k^{\prime} \lambda^{\prime}} a_{k, \lambda}^{\dagger} a_{k^{\prime}, \lambda^{\prime}}+\frac{1}{2} \tilde{B}_{k \lambda, k^{\prime} \lambda^{\prime}} a_{k, \lambda} a_{k^{\prime}, \lambda^{\prime}}+\frac{1}{2} \tilde{B}_{k \lambda, k^{\prime} \lambda^{\prime}}^{\dagger} a_{k, \lambda}^{\dagger} a_{k^{\prime}, \lambda^{\prime}}^{\dagger}\right)-\right.} \\
\left.\quad-\sum_{k, \lambda}\left(\tilde{D}_{k, \lambda} a_{k, \lambda}+\tilde{D}_{k, \lambda}^{\dagger} a_{k, \lambda}^{\dagger}\right)+\sum_{k, \lambda} \frac{\delta}{2(n \cdot p) \omega_{k}}-R\right] F=0
\end{gathered}
$$

onde,

$$
\begin{aligned}
\tilde{A}_{k \lambda, k^{\prime} \lambda^{\prime}} & =\omega_{k} \delta_{k k^{\prime}} \delta_{\lambda \lambda^{\prime}}+\frac{\delta\left(\boldsymbol{e}_{\lambda}^{*} \cdot \boldsymbol{e}_{\lambda^{\prime}}\right)}{(n \cdot p) \sqrt{\omega_{k} \omega_{k^{\prime}}}} \\
\tilde{B}_{k \lambda, k^{\prime} \lambda^{\prime}} & =\frac{\delta\left(\boldsymbol{e}_{\lambda} \cdot \boldsymbol{e}_{\lambda^{\prime}}\right)}{(n \cdot p) \sqrt{\omega_{k} \omega_{k^{\prime}}}} \\
\tilde{D}_{k, \lambda} & =\epsilon \sqrt{\frac{\delta}{\omega_{k}}} \frac{\left(\boldsymbol{p} \cdot \boldsymbol{e}_{\lambda}\right)}{(n \cdot p)}
\end{aligned}
$$

Podemos reduzir a (2.2.15) a uma forma diagonal com relação aos índices de polarização. Para tanto, introduzimos outra base de vetores de polarização, a base de polarização linear $\left\{\varepsilon_{\sigma}, \sigma=\right.$ $1,2\}$ que se relaciona com os vetores de polarização circular através de uma transformação 
linear:

$$
\boldsymbol{e}_{\lambda}=\sum_{\sigma} \alpha_{\lambda \sigma} \varepsilon_{\sigma}, \quad \boldsymbol{e}_{\lambda}^{*}=\sum_{\sigma} \alpha_{\lambda \sigma}^{*} \varepsilon_{\sigma}, \quad \alpha=\frac{1}{\sqrt{2}}\left(\begin{array}{cc}
1 & i \\
i & 1
\end{array}\right),
$$

e satisfaz as seguintes propriedades:

$$
\boldsymbol{\varepsilon}_{\sigma} \cdot \boldsymbol{\varepsilon}_{\sigma^{\prime}}=\delta_{\sigma \sigma^{\prime}}, \quad \boldsymbol{\varepsilon}_{\sigma} \cdot \boldsymbol{n}=0, \quad \boldsymbol{\varepsilon}_{1} \times \boldsymbol{\varepsilon}_{2}=\boldsymbol{n}, \quad \sigma=1,2 .
$$

Usando essas propriedades, podemos escrever $\alpha_{\lambda \sigma}$ e $\alpha_{\lambda \sigma}^{*}$ como:

$$
\alpha_{\lambda \sigma}=e_{\lambda} \cdot \varepsilon_{\sigma}, \quad \alpha_{\lambda \sigma}^{*}=e_{\lambda}^{*} \cdot \varepsilon_{\sigma}
$$

A transformação inversa é da forma:

$$
\boldsymbol{\varepsilon}_{\sigma}=\sum_{\lambda} \beta_{\sigma \lambda} \boldsymbol{e}_{\lambda} \Rightarrow \beta_{\sigma \lambda}=\varepsilon_{\sigma} \cdot \boldsymbol{e}_{\lambda}^{*}=\alpha_{\sigma \lambda}^{*}
$$

Observe que

$$
\begin{aligned}
\boldsymbol{e}_{\lambda} & =\sum_{\sigma} \alpha_{\lambda \sigma} \varepsilon_{\sigma}=\sum_{\sigma} \alpha_{\lambda \sigma}\left(\sum_{\lambda^{\prime}} \alpha_{\sigma \lambda^{\prime}}^{*} \boldsymbol{e}_{\lambda^{\prime}}\right) \\
& =\sum_{\lambda^{\prime}}\left(\sum_{\sigma} \alpha_{\lambda \sigma} \alpha_{\sigma \lambda^{\prime}}^{*}\right) \boldsymbol{e}_{\lambda^{\prime}}
\end{aligned}
$$

portanto,

$$
\sum_{\sigma} \alpha_{\lambda \sigma} \alpha_{\sigma \lambda^{\prime}}^{*}=\delta_{\lambda \lambda^{\prime}}
$$

i.e. a matrize $\alpha$ é hermitiana. A matriz $\alpha$ ainda exerce o papel de realizar a transformação canônica que relaciona operadores de criação e aniquilação associados à polarização circular, $a_{k, \lambda}^{\dagger}$ e $a_{k, \lambda}$, com aqueles associados à polarização linear, que denotaremos $b_{k, \sigma}^{\dagger}$ e $b_{k, \sigma}$, i.e.:

$$
a_{k, \lambda}=\sum_{\sigma} \alpha_{\lambda \sigma} b_{k, \sigma}, \quad a_{k, \lambda}^{\dagger}=\sum_{\sigma} \alpha_{\sigma \lambda}^{*} b_{k, \sigma}^{\dagger}
$$


Fazendo a mudança de vetores de polarização (2.2.16) na equação (2.2.15) e usando (2.2.17), obtemos:

$$
\begin{aligned}
& {\left[\sum_{k, k^{\prime}, \sigma}\left(A_{k k^{\prime}} b_{k, \sigma}^{\dagger} b_{k^{\prime}, \sigma}+\frac{1}{2} B_{k k^{\prime}} b_{k, \sigma} b_{k^{\prime}, \sigma}+\frac{1}{2} B_{k k^{\prime}}^{\dagger} b_{k, \sigma}^{\dagger} b_{k^{\prime}, \sigma}^{\dagger}\right)-\right.} \\
& \left.-\sum_{k, \lambda} D_{k, \sigma}\left(b_{k, \sigma}+b_{k, \sigma}^{\dagger}\right)+\frac{\delta}{(n \cdot p)} \sum_{k} \frac{1}{\omega_{k}}-R\right] F=0
\end{aligned}
$$

onde

$$
\begin{aligned}
A_{k k^{\prime}} & =\omega_{k} \delta_{k k^{\prime}}+\frac{\delta}{(n \cdot p) \sqrt{\omega_{k} \omega_{k^{\prime}}}}, \\
B_{k k^{\prime}} & =\frac{\delta}{(n \cdot p) \sqrt{\omega_{k} \omega_{k^{\prime}}}} \\
D_{k, \sigma} & =\epsilon \sqrt{\frac{\delta}{\omega_{k}}} \frac{\left(\boldsymbol{p} \cdot \boldsymbol{\varepsilon}_{\sigma}\right)}{(n \cdot p)} .
\end{aligned}
$$

Nosso próximo passo consiste em considerar uma transformação canônica

$$
b_{k, \sigma}=\sum_{l=1}^{\infty}\left(\phi_{k l} c_{l, \sigma}+\psi_{k l} c_{l, \sigma}^{\dagger}\right)+\gamma_{k, \sigma}
$$

que transforme a (2.2.18) em

$$
\left(\sum_{k, \sigma} r_{k} c_{k, \sigma}^{\dagger} c_{k, \sigma}-R_{0}\right) F=0
$$

Devemos notar que a (2.2.18) possui apenas coeficientes reais, de forma que podemos supor que os coeficientes de (2.2.19) sejam reais. Tendo em vista as condições (C.0.4)-(C.0.6) apresentadas no apêndice $\mathrm{C}$ sobre sistemas com Hamiltonianos quadráticos, afirmamos que a condição para que a (2.2.18) possa ser escrita na forma (2.2.20) é:

$$
\begin{aligned}
r_{k} \phi_{s k} & =\sum_{l}\left(A_{s l} \phi_{l k}+B_{s l} \psi_{l k}\right), \\
-r_{k} \psi_{s k} & =\sum_{l}\left(A_{s l} \psi_{l k}+B_{s l} \phi_{l k}\right), \\
D_{s, \sigma} & =\sum_{l}\left(A_{s l}+B_{s l}\right) \gamma_{l, \sigma} .
\end{aligned}
$$


Em [2] é apresentada a solução do sistema de equações acima e o resultado é:

$$
\begin{aligned}
\phi_{s k} & =\frac{1}{2}\left[\left(\frac{\omega_{s}}{r_{k}}\right)^{1 / 2}+\left(\frac{r_{k}}{\omega_{s}}\right)^{1 / 2}\right] q_{s k}, \\
\psi_{s k} & =\frac{1}{2}\left[\left(\frac{\omega_{s}}{r_{k}}\right)^{1 / 2}-\left(\frac{r_{k}}{\omega_{s}}\right)^{1 / 2}\right] q_{s k}, \\
q_{s k} & =\frac{1}{\left(r_{k}^{2}-\omega_{s}^{2}\right)}\left[\sum_{l}\left(r_{k}^{2}-\omega_{l}^{2}\right)^{-2}\right]^{-1 / 2}, \\
\gamma_{s, \sigma} & =\epsilon \sqrt{\frac{\delta}{\omega_{s}^{3}}} \frac{\left(\boldsymbol{p} \cdot \boldsymbol{\varepsilon}_{\sigma}\right)}{[(n \cdot p)+\nu \delta]}, \quad \nu=\sum_{k} \frac{1}{\omega_{k}^{2}}
\end{aligned}
$$

Os $r_{k}$ são enumerados de forma crescente e são as raízes da equação

$$
\sum_{s} \frac{1}{r_{k}^{2}-\omega_{s}^{2}}=\frac{(n \cdot p)}{\delta} \equiv \frac{2(n \cdot p)}{\rho \alpha}
$$

A constante presente em (2.2.20) é dada por

$$
R_{0}=R-\Omega+\frac{\nu \delta p_{\perp}^{2}}{(n \cdot p)[(n \cdot p)+\nu \delta]}, \quad p_{\perp}^{2}=\sum_{\sigma}\left(\boldsymbol{p} \cdot \boldsymbol{\varepsilon}_{\sigma}\right)^{2}, \quad \Omega=\sum_{s}\left(r_{s}-\omega_{s}\right) .
$$

A solução de (2.2.20) é, consequentemente, da forma

$$
F=\prod_{k, \sigma} \frac{\left(c_{k, \sigma}^{\dagger}\right)^{N_{k, \sigma}}}{\sqrt{N_{k, \sigma}}}|0\rangle
$$

onde $N_{k, \sigma}$ é um inteiro que representa a população do estado com números quânticos $\{k, \sigma\}$ e que nos permite escrever o autovalor $R$ como

$$
R=\sum_{k, \sigma} r_{k} N_{k, \sigma}+\Omega-\frac{\nu \delta p_{\perp}^{2}}{(n \cdot p)[(n \cdot p)+\nu \delta]}
$$

Para uso futuro, tendo em mente a expressão (B.0.8) do apêndice sobre transformações canônicas lineares, escrevemos o operador $c_{k, \sigma}$ em termos de $b_{l, \sigma}$ e $b_{l, \sigma}^{\dagger}$ :

$$
c_{k, \sigma}=\phi_{l k} b_{l, \sigma}-\psi_{l k} b_{l, \sigma}^{\dagger}+\left(\psi_{l k}-\phi_{l k}\right) \gamma_{l, \sigma}
$$




\subsection{Solução Exata em Presença de um Campo Magnético Externo Constante e Homogêneo}

Baseados em [6] e [7], mostraremos nesta seção como a presença de um campo magnético externo $B$ constante e homogêneo, fixado na direção de propagação dos fótons, altera os operadores de criação e aniquilação de quasifótons. Não daremos todos os detalhes da dedução formal, para os quais indicamos [6] e [7], mas apenas o resultado final e uma breve discussão.

A equação (4.1.1) é modificada da seguinte forma:

$$
b_{k \lambda}=\sum_{s=0}^{\infty} \sum_{\lambda=1,2}\left(\phi_{k \lambda, s \lambda^{\prime}} c_{s \lambda^{\prime}}+\psi_{k \lambda, s \lambda^{\prime}}^{*} c_{s \lambda^{\prime}}^{\dagger}\right)+\gamma_{k \lambda},
$$

onde os índices $k$ e $s$ estão associados às frequências dos fótons e $\lambda$ e $\lambda^{\prime}$ às polarizações. A primeira modificação que verificamos é que agora os operadores de criação e aniquilação de fótons $b_{k \lambda}^{\dagger}$ e $b_{k \lambda}$ misturam as polarizações dos quasifótons, o que não ocorria na ausência do campo magnético. Além disse, como nota [6], o movimento ciclotrônico do elétron é descrito

pela presença de um estado de quasifóton adicional associado a $s=0 .{ }^{*}$ Os coeficientes $\phi_{k \lambda, s \lambda^{\prime}}$, $\psi_{k \lambda, s \lambda^{\prime}}$ e $\gamma_{k \lambda}$ são dados por:

$$
\begin{aligned}
& \phi_{k \lambda, s \lambda^{\prime}}=\left\{\left[\left(\frac{\omega_{k}}{r_{s \lambda^{\prime}}}\right)^{1 / 2}+\left(\frac{r_{s \lambda^{\prime}}}{\omega_{k}}\right)^{1 / 2}\right] \frac{\left(3-2 \lambda^{\prime}\right)(2-\lambda)-i(\lambda-1)}{2\left(r_{s \lambda^{\prime}}^{2}-\omega_{k}^{2}\right)}\left(1-\delta_{0 k}\right)+\right. \\
& \left.+(\lambda-1)\left(\lambda^{\prime}-1\right)\left[\frac{(n p) E_{0}}{\alpha \rho} \frac{1}{r_{s \lambda^{\prime}}^{3}}\right]^{1 / 2} \delta_{0 s}\right\}\left[1+\left(\lambda^{\prime}-2\right) \delta_{0 s}\right] S_{s \lambda^{\prime}} \\
& \psi_{k \lambda, s \lambda^{\prime}}=\left\{\left[\left(\frac{\omega_{k}}{r_{s \lambda^{\prime}}}\right)^{1 / 2}-\left(\frac{r_{s \lambda^{\prime}}}{\omega_{k}}\right)^{1 / 2}\right] \frac{\left(3-2 \lambda^{\prime}\right)(2-\lambda)-i(\lambda-1)}{2\left(r_{s \lambda^{\prime}}^{2}-\omega_{k}^{2}\right)}\left(1-\delta_{0 k}\right)+\right. \\
& \left.+(\lambda-1)\left(2-\lambda^{\prime}\right)\left[\frac{(n p) E_{0}}{\alpha \rho} \frac{1}{r_{s \lambda^{\prime}}^{3}}\right]^{1 / 2} \delta_{0 k}\right\}\left[1+\left(\lambda^{\prime}-2\right) \delta_{0 s}\right] S_{s \lambda^{\prime}} \\
& S_{s \lambda^{\prime}}=\left[\frac{\left(2 \lambda^{\prime}-3\right)(n p) E_{0}}{\alpha \rho} \frac{1}{r_{s \lambda^{\prime}}^{3}}+2 \sum_{j}\left(r_{s \lambda^{\prime}}^{2}-\omega_{j}^{2}\right)^{-2}\right]^{1 / 2} \\
& =\left[(2 \lambda-3) \frac{\alpha \rho}{(n p)} r_{s \lambda^{\prime}}^{3} \frac{\partial r_{s \lambda^{\prime}}}{\partial E_{0}}\right]^{1 / 2} \text {, } \\
& \gamma_{k \lambda}=-\left(p e_{1}\right)(\lambda-1)\left[2 E_{0}(n p)\right]^{-1 / 2} \delta_{0 k}, \quad E_{0}=\frac{|e| B}{(n p)},
\end{aligned}
$$

${ }^{*} \mathrm{O}$ operador aniquilação de fótons $b_{k \lambda}$ é definido de forma que quando $k=0$ só exista uma polarização possível, o mesmo ocorre pros operadores de aniquilação de quasifótons $c_{s \lambda^{\prime}}$. 
onde os $r_{k \lambda}$ são as raízes da equação:

$$
\frac{\alpha \rho}{(n p)} \sum_{s}\left(r_{k \lambda}^{2}-\omega_{s}^{2}\right)^{-1}=1+(3-2 \lambda) \frac{E_{0}}{r_{k \lambda}}, \quad r_{01}=0
$$




\section{Capítulo 3}

\section{Emaranhamento em Sistemas Bipartidos de Dimensão Finita}

Um sistema quântico dito bipartido é tal que seu espaço de Hilbert $\mathcal{H}$ pode ser decomposto como o produto tensorial de dois outros espaços, i.e. $\mathcal{H}=\mathcal{H}_{A} \otimes \mathcal{H}_{B}$. Neste caso, dizemos que o sistema descrito no espaço de Hilbert $\mathcal{H}$ tem dois subsistemas, o subsistema $A$, descrito no espaço de Hilbert $\mathcal{H}_{A}$ e o subsistema $B$, descrito no subsistema $\mathcal{H}_{B}$.

Em particular, estamos interessados em sistemas de dimensão finita, i.e. $\operatorname{dim} \mathcal{H}<\infty$. Como $\operatorname{dim} \mathcal{H}=\operatorname{dim} \mathcal{H}_{A} \operatorname{dim} \mathcal{H}_{B}$, então $\operatorname{dim} \mathcal{H}_{A}, \operatorname{dim} \mathcal{H}_{B}<\infty$. Sistemas de dimensão finita aparecem frequentemente na mecânica quântica, pois há situações em que temos um sistema complexo que apresenta um espectro de energia discreto porém na situação dinâmica que ele se encontra apenas alguns desses níveis de energia são relevantes. Nesses casos é frequente, como diz [21], o uso de uma aproximação esquemática em que apenas um número finito de níveis são considerados. Para ilustrar tal afirmação podemos citar o caso da interação ressonante de dois estados estacionários atômicos com o campo eletromagnético de uma cavidade, permitindo ignorar a participação de outros níveis não ressonantes [16].

Por questão de referência futura, abordaremos brevemente neste capítulo a questão do emranhamento em dimensão infinita. Enfatizamos que esse tópico não será utilizado no desenvolvimento do trabalho.

Antes de falarmos sobre o conceito de emaranhamento convém apresentarmos o formalismo da matriz densidade que será de grande importância na teoria do emaranhamento.

\subsection{Formalismo da Matriz Densidade}

A matriz densidade providencia uma forma conveniente de tratar sistemas cujo estado não pode ser descrito por uma função de onda, o que ocorre quando introduzimos na teoria quân- 
tica a possibilidade do sistema apresentar incertezas estatísticas de origem clássica. Suponha um conjunto de estados $\left\{\left|\psi_{i}\right\rangle\right\}$ e suponha que um sistema quântico está em algum dos estados $\left|\psi_{i}\right\rangle$ com probabilidade $p_{i}$. Chamamos o conjunto $\left\{p_{i},\left|\psi_{i}\right\rangle\right\}, \sum_{i} p_{i}=1$, um conjunto de estados puros. A matriz densidade desse sistema é dada por:

$$
\rho=\sum_{i} p_{i}\left|\psi_{i}\right\rangle\left\langle\psi_{i}\right|
$$

\subsubsection{Propriedades Gerais da Matriz Densidade}

Definição. Um operador $\rho$ é a matriz densidade associada a algum conjunto $\left\{p_{i},\left|\psi_{i}\right\rangle\right\}$, $\sum_{i} p_{i}=1$ se e somente se satisfaz as condições:

1. (Condição do Traço): $\operatorname{tr}(\rho)=1$.

2. (Positividade) $\rho$ é um operador positivo.

O formalismo da matriz densidade nos fornece uma nova representação para a Mecânica Quântica, de forma que podemos reescrever seus postulados nessa nova linguagem:

Postulado 1 Associamos a qualquer sistema físico isolado um espaço de Hilbert $\mathcal{H}$, denominado espaço de estados do sistema. O sistema é completamente descrito pela sua matriz densidade, que é um operador positivo $\rho$ de traço 1 agindo em $\mathcal{H}$.

Postulado 2 A evolução de um sistema quântico fechado é descrita por uma transformação unitária. Dessa forma, o estado $\rho$ do sistema no instante $t_{1}$ é relacionado ao estado $\rho^{\prime}$ no instante $t_{2}$ por uma transformação unitária $U$ que depende apenas dos instantes $t_{1}$ e $t_{2}$,

$$
\rho^{\prime}=U \rho U^{\dagger}
$$

Postulado 3 Medições quânticas são descritas por uma coleção $\left\{M_{m}\right\}$ de operadores de medição agindo em $\mathcal{H}$. $\mathrm{O}$ índice $m$ se refere ao resultado de medida que pode ocorrer no experimento. Se o estado do sistema é $\rho$ imediatamente antes da medida, então a probabilidade de que o resultado $m$ ocorra é dada por:

$$
p(m)=\operatorname{tr}\left(M_{m}^{\dagger} M_{m} \rho\right)
$$


e o estado do sistema após a medida é

$$
\frac{M_{m} \rho M_{m}^{\dagger}}{\operatorname{tr}\left(M_{m}^{\dagger} M_{m} \rho\right)}
$$

Os operadores de medição satisfazem a relação de completeza:

$$
\sum_{m} M_{m}^{\dagger} M_{m}=\mathbb{I}
$$

Postulado 4 O espaço de estados de um sistema composto é o produto tensorial dos espaços de estados dos sistemas componentes. Para um sistema composto de $n$ partes, se cada uma dessas partes é descrita por uma matriz de densidade $\rho_{i}, 1 \leq i \leq n$, então a matriz densidade que descreve o sistema composto é $\rho=\rho_{1} \otimes \cdots \otimes \rho_{n}$.

Definição. Dizemos que a matriz densidade representa um estado puro quando o conjunto $\left\{p_{i},\left|\psi_{i}\right\rangle\right\}$ só apresenta um único elemento $|\psi\rangle$, obviamente $p=1$ nesse caso. O caso geral representa uma mistura.

Proposição. Dada uma matriz densidade $\rho, \operatorname{tr}\left(\rho^{2}\right) \leq 1$, de forma que a igualdade é verificada se e somente se $\rho$ representa um estado puro.

Demonstração. Seja

$$
\rho=\sum_{i} p_{i}\left|\psi_{i}\right\rangle\left\langle\psi_{i}\right|
$$

então

$$
\rho^{2}=\sum_{i, j} p_{i} p_{j}\left|\psi_{i}\right\rangle\left\langle\psi_{i} \mid \psi_{j}\right\rangle\left\langle\psi_{j}\right|
$$

Tomando o traço de $\rho^{2}$, obtemos

$$
\begin{aligned}
\operatorname{tr}\left(\rho^{2}\right) & =\sum_{i, j, k} p_{i} p_{j}\left\langle k \mid \psi_{i}\right\rangle\left\langle\psi_{i} \mid \psi_{j}\right\rangle\left\langle\psi_{j} \mid k\right\rangle \\
& =\sum_{i, j} p_{i} p_{j} \underbrace{\left|\left\langle\psi_{i} \mid \psi_{j}\right\rangle\right|^{2}}_{\leq 1} \\
& \leq\left(\sum_{i} p_{i}\right)^{2}=1,
\end{aligned}
$$

onde os vetores $|k\rangle$ formam uma base para o espaço de estados. Dessa forma, obtemos que 
$\operatorname{tr}\left(\rho^{2}\right) \leq 1$. No caso particular de um estado puro, i.e. $\rho=|\psi\rangle\langle\psi|$, obtemos

$$
\rho^{2}=\rho \Rightarrow \operatorname{tr}\left(\rho^{2}\right)=\operatorname{tr}(\rho)=1 \text {. }
$$

Com isso mostramos que se $\rho$ representa um estado puro, então $\operatorname{tr}\left(\rho^{2}\right)=1$. Por outro lado, para que $\operatorname{tr}\left(\rho^{2}\right)=1$ devemos ter que $\left\langle\psi_{i} \mid \psi_{j}\right\rangle=1, \forall i, j$. Isso nos leva a concluir que $\left|\psi_{i}\right\rangle=\left|\psi_{j}\right\rangle=|\psi\rangle, \forall i, j$, i.e. $\rho=|\psi\rangle\langle\psi|$.

\subsubsection{A Matriz Densidade Reduzida}

Definição. Sejam $\mathcal{H}_{A}$ e $\mathcal{H}_{B}$ dois espaços vetoriais e $L\left(\mathcal{H}_{A}\right)$ e $L\left(\mathcal{H}_{B}\right)$ espaços de operadores lineares definidos em $\mathcal{H}_{A}$ e $\mathcal{H}_{B}$, respectivamente. Definimos a operação traço parcial sobre o espaço $\mathcal{H}_{B}$ como sendo a operação que satisfaz:

$$
\begin{gathered}
\operatorname{tr}_{B}: L\left(\mathcal{H}_{A} \otimes \mathcal{H}_{B}\right) \rightarrow L\left(\mathcal{H}_{A}\right), \rho_{A} \otimes \rho_{B} \mapsto \rho_{A} \operatorname{tr}\left(\rho_{B}\right) \\
\operatorname{tr}_{B}\left(\alpha \rho_{A} \otimes \rho_{B}+\beta \sigma_{A} \otimes \sigma_{B}\right)=\alpha \rho_{A} \operatorname{tr}\left(\rho_{B}\right)+\beta \sigma_{A} \operatorname{tr}\left(\sigma_{B}\right), \alpha, \beta \in \mathbb{C} .
\end{gathered}
$$

De forma análoga, definimos o traço parcial em relação a $\mathcal{H}_{A}$.

Suponha um sistema composto de duas partes, $A$ e $B$, cuja matriz densidade seja $\rho_{A B}$. Podemos obter a matriz densidade que descreve cada um dos subsistemas $A$ e $B$ a partir de $\rho_{A B}$ usando o traço parcial.Como exemplo, considere o estado $|\psi\rangle=(|00\rangle+|11\rangle) / \sqrt{2}$. A matriz densidade associada a esse estado é

$$
\begin{aligned}
\rho_{A B} & =|\psi\rangle\langle\psi|=\left(\frac{|00\rangle+|11\rangle}{\sqrt{2}}\right)\left(\frac{\langle 00|+\langle 11|}{\sqrt{2}}\right) \\
& =\frac{|00\rangle\langle 00|+| 00\rangle\langle 11|+| 11\rangle\langle 00|+| 11\rangle\langle 11|}{2} .
\end{aligned}
$$

Obviamente essa matriz densidade representa um estado puro e não uma mistura, i.e. $\operatorname{tr}\left(\rho^{2}\right)=$ 1. A matriz densidade $\rho_{A}$ que descreve o subsistema $A$ é dada por:

$$
\begin{aligned}
\rho_{A} & =\operatorname{tr}_{B}\left(\rho_{A B}\right) \\
& =\frac{\operatorname{tr}_{B}(|00\rangle\langle 00|)+\operatorname{tr}_{B}(|00\rangle\langle 11|)+\operatorname{tr}_{B}(|11\rangle\langle 00|)+\operatorname{tr}_{B}(|11\rangle\langle 11|)}{2} \\
& =\frac{|0\rangle\langle 0|\langle 0 \mid 0\rangle+| 0\rangle\langle 1|\langle 0 \mid 1\rangle+| 1\rangle\langle 0|\langle 1 \mid 0\rangle+| 1\rangle\langle 1|\langle 1 \mid 1\rangle}{2} \\
& =\frac{|0\rangle\langle 0|+| 1\rangle\langle 1|}{2}=\frac{1}{2} \mathbb{I} .
\end{aligned}
$$


Perceba que $\rho_{A}$ não representa um estado puro, visto que $\operatorname{tr}\left(\rho_{A}^{2}\right)=\operatorname{tr}\left((\mathbb{I} / 2)^{2}\right)=1 / 2<1$ e, além disso, em geral, $\rho_{A B} \neq \rho_{A} \otimes \rho_{B}$.

\subsubsection{A Distribuição de Wigner}

O texto que segue é baseado na exposição sobre o assunto feita em [15] e tem como objetivo introduzir a noção de distribuição de Wigner que será útil posteriormente. No artigo [26], Wigner formula a mecânica quântica em termos de uma função $W(\boldsymbol{q}, \boldsymbol{p})$ definida no espaço de fase de forma que tal função consegue reproduzir corretamente as distribuições de probabilidade para $\boldsymbol{q}$ e $\boldsymbol{p}$. O interesse dessa reformulação reside no fato de que ela nos fornece uma expressão da mecânica quântica em termos de conceitos clássicos, com efeito, nessa formulação, valores esperados da mecânica quântica se tornam médias no espaço de fase segundo uma certa distribuição.

Considere um sistema $n$-dimensional com matriz densidade $\hat{\rho}$. A distribuição de Wigner associada a $\hat{\rho}$ é definida da seguinte forma:

$$
W(\boldsymbol{q}, \boldsymbol{p})=\frac{1}{\pi^{n}} \int d^{n} q^{\prime} e^{2 i \boldsymbol{p} \cdot \boldsymbol{q}^{\prime}}\left\langle\boldsymbol{q}-\boldsymbol{q}^{\prime}|\hat{\rho}| \boldsymbol{q}+\boldsymbol{q}^{\prime}\right\rangle
$$

A função de Wigner $W(\boldsymbol{q}, \boldsymbol{p})$ tem três propriedades que coincidem com as propriedades de uma distribuição clássica no espaço de fase, a saber:

1. Como $\hat{\rho}$ é hermitiano, então $W(\boldsymbol{q}, \boldsymbol{p})$ é real.

2. Integração de $W(\boldsymbol{q}, \boldsymbol{p})$ sobre $\boldsymbol{p}$ fornece a distribuição de probabilidade de $\boldsymbol{q}$ :

$$
\int d^{n} p W(\boldsymbol{q}, \boldsymbol{p})=\langle\boldsymbol{q}|\hat{\rho}| \boldsymbol{q}\rangle
$$

3. Integração de $W(\boldsymbol{q}, \boldsymbol{p})$ sobre $\boldsymbol{q}$ fornece a distribuição de probabilidade de $\boldsymbol{p}$ :

$$
\int d^{n} q W(\boldsymbol{q}, \boldsymbol{p})=\langle\boldsymbol{p}|\hat{\rho}| \boldsymbol{p}\rangle
$$

Apesar dessas propriedades, $W(\boldsymbol{q}, \boldsymbol{p})$ não é uma distribuição de probabilidade porque não é positivo definido para um estado $\hat{\rho}$ arbitrário.

Podemos também associar observáveis em mecânica quântica a funções no espaço de fase. Seja $\hat{A}$ um observável e seja uma função $\mathcal{A}(\boldsymbol{q}, \boldsymbol{p})$ tal que:

$$
\int d^{n} q d^{n} p \mathcal{A}(\boldsymbol{q}, \boldsymbol{p})=\operatorname{tr}(\hat{A} \hat{\rho})
$$


Pode-se verificar que $\mathcal{A}(\boldsymbol{q}, \boldsymbol{p})$ admite a seguinte expressão:

$$
\mathcal{A}(\boldsymbol{q}, \boldsymbol{p})=\frac{1}{\pi^{n}} \int d^{n} q^{\prime} e^{2 i \boldsymbol{p} \cdot \boldsymbol{q}^{\prime}}\left\langle\boldsymbol{q}-\boldsymbol{q}^{\prime}|\hat{A}| \boldsymbol{q}+\boldsymbol{q}^{\prime}\right\rangle
$$

Seguindo a definição apresentada em [24], dizemos que um estado $\hat{\rho}$ representa um estado gaussiano se sua distribuição de Wigner for uma gaussiana no espaço de fase:

$$
W(\xi)=\frac{1}{4 \pi^{2} \sqrt{\operatorname{det} V}} e^{-\frac{1}{2} \xi^{t} V^{-1} \xi}
$$

onde $\xi=\left(q_{1}, p_{1}, \ldots, q_{n}, p_{n}\right)$ e $V$ é a matriz de correlações.

\subsection{Critério de Separabilidade}

Definição (Emaranhamento). Dizemos que um estado $|\psi\rangle \in \mathcal{H}=\mathcal{H}_{1} \otimes \cdots \otimes \mathcal{H}_{n}$ é não emaranhado (ou separado) se e somente se $|\psi\rangle$ puder ser escrito como:

$$
|\psi\rangle=\left|\psi_{1}\right\rangle \otimes \cdots \otimes\left|\psi_{n}\right\rangle,\left|\psi_{k}\right\rangle \in \mathcal{H}_{k}, k \in\{1, \ldots, n\}
$$

A definição de emaranhamento é muito clara e simples porém nada prática. Um grande é problema é o de decidir se um dado estado $|\psi\rangle$ é ou não emaranhado. No que segue vamos discutir essa questão no caso de estados puros, bipartidos em dimensão finita.

\subsubsection{Estados Puros Bipartidos em Dimensão Finita: Decomposição de Schmidt}

Para o que segue faremos uso de dois resultados de álgebra linear, a decomposição polar e a decomposição de valor singular. As demonstrações aqui apresentadas são baseadas nas demonstrações presentes em [9].

Teorema (Decomposição Polar). Seja A um operador num espaço vetorial $V$. Existe um operador unitário $U$ e um operador positivo $J$ tal que

$$
A=U J
$$

onde o único operador positivo $J$ que satisfaz essa equação é $J=\sqrt{A^{\dagger} A}$. No caso de $A$ ser inversivel, então $U$ é única.

Demonstração. Seja $A \in \operatorname{Mat}(\mathbb{C}, n)$ uma matriz $n \times n$ com entradas complexas. Claramente a matriz $A^{\dagger} A$ é hermitiana. Pelo teorema espectral, existe uma base de $n$ vetores ortonormais 
que satisfazem:

$$
A^{\dagger} A v_{k}=d_{k} v_{k}, \quad k=1, \ldots, n
$$

e uma matriz de passagem $P$ formada pelos autovetores de $A^{\dagger} A$, i.e. $P=\left[v_{1}, \ldots, v_{n}\right]$, tal que $P^{\dagger}\left(A^{\dagger} A\right) P=D:=\operatorname{diag}\left(d_{1}, \ldots, d_{n}\right)$. Observe que os $d_{k}$ são não negativos:

$$
\left\|A v_{k}\right\|^{2}=\left\langle A v_{k} \mid A v_{k}\right\rangle=\left\langle v_{k} \mid A^{\dagger} A v_{k}\right\rangle=d_{k}\left\|v_{k}\right\|^{2} \Rightarrow d_{k}=\frac{\left\|A v_{k}\right\|^{2}}{\left\|v_{k}\right\|^{2}} \Rightarrow d_{k} \geq 0 .
$$

Definimos $D^{1 / 2}:=\operatorname{diag}\left(\sqrt{d_{1}}, \ldots, \sqrt{d_{n}}\right)$. Os números não negativos $\sqrt{d_{1}}, \ldots, \sqrt{d_{n}}$ são os chamados valores singulares da matriz $A$. Definimos

$$
\sqrt{A^{\dagger} A}=P D^{1 / 2} P^{\dagger}
$$

Perceba que

$$
\left(\operatorname{det} \sqrt{A^{\dagger} A}\right)^{2}=\operatorname{det}\left(A^{\dagger} A\right)=|\operatorname{det} A|^{2},
$$

o que nos diz que a matriz $\sqrt{A^{\dagger} A}$ é inversível se e somente se a matriz $A$ o for.

Considere o conjunto $\left\{d_{k}, k=1, \ldots, n\right\}$ de autovalores de $A^{\dagger} A$ e suponha um ordenamento desses autovalores de forma que para $k=1, \ldots, l, d_{k}>0$ e para $k=l+1, \ldots, n, d_{k}=0$. Para $k=1, \ldots, l$, sejam $w_{k}$ vetores definidos como:

$$
w_{k}:=\frac{1}{\sqrt{d_{k}}} A v_{k}, \quad k=1, \ldots, l
$$

Claramente esses vetores são ortonormais:

$$
\left\langle w_{p} \mid w_{q}\right\rangle=\frac{1}{\sqrt{d_{p} d_{q}}}\left\langle A v_{p} \mid A v_{q}\right\rangle=\frac{1}{\sqrt{d_{p} d_{q}}}\left\langle v_{p} \mid A^{\dagger} A v_{q}\right\rangle=\frac{d_{n}}{\sqrt{d_{m} d_{n}}}\left\langle v_{p} \mid v_{q}\right\rangle=\delta_{p q}
$$

O conjunto $\left\{w_{k}, k=1, \ldots, l\right\}$ é um conjunto ortonormal que pode ser completado com mais $n-l$ vetores ortonormais $\left\{w_{k}, k=l+1, \ldots, n\right\}$. Seja $Q$ a matriz cujas colunas são dadas pelos $w_{k}$, i.e. $Q=\left[w_{1}, \ldots, w_{n}\right]$. Definimos

$$
U:=Q P^{\dagger}
$$

Como os conjuntos $\left\{v_{k}, k=1, \ldots, n\right\}$ e $\left\{w_{k}, k=1, \ldots, n\right\}$ são ortonormais, então as matriz $Q$ e $P$ são unitárias e consequentemente $U$ é uma matriz unitária. Agora vamos mostrar que 
$A P=Q D^{1 / 2}$

$$
\begin{aligned}
A P & =A\left[v_{1}, \ldots, v_{n}\right]=\left[A v_{1}, \ldots, A v_{n}\right]=\left[\sqrt{d_{1}} w_{1}, \ldots, \sqrt{d_{l}} w_{l}, 0, \ldots, 0\right] \\
& =\left[\sqrt{d_{1}}, \ldots, \sqrt{d_{n}}\right] D^{1 / 2}=Q D^{1 / 2} .
\end{aligned}
$$

Agora, dado que $A P=Q D^{1 / 2}$, segue que $A=Q D^{1 / 2} P^{\dagger}=U P D^{1 / 2} P^{\dagger}=U \sqrt{A^{\dagger} A}$, como queríamos mostrar. Para mostrar a unicidade da matriz $U$ considere que existe uma matriz $U^{\prime}$ tal que $A=U \sqrt{A^{\dagger} A}=U^{\prime} \sqrt{A^{\dagger} A}$. Como vimos, $\sqrt{A^{\dagger} A}$ é inversível se e somente se $A$ for inversível. Dado que $A$ é inversível, temos claramente que $U=U^{\prime}$, caso contrário a matriz unitária $U$ não é única e a arbitrariedade está na escolha do complemento ortogonal $\left\{w_{k}, k=l+1, \ldots, r\right\}$.

Corolário (Decomposição de Valor Singular para Matrizes Quadradas). Seja $A \in$ Mat $(\mathbb{C}, \mathrm{n})$ uma matriz quadrada de coeficientes complexos. Existem matrizes unitárias $U, V \in$ Mat $(\mathbb{C}, n)$ e também uma matriz diagonal $D$ com entradas não negativas de forma que

$$
A=U D V
$$

Os elementos diagonais de $D$ são chamados valores singulares de $A$.

Demonstração. Pela decomposição polar, $A=S J$, onde $S$ é unitária e $J$ positiva. Como todo operador positivo é hermitiano e consequentemente normal, então pelo teorema espectral $J=T D T^{\dagger}$, onde $T$ é unitária e $D$ é diagonal com entradas não negativas. Dessa forma, obtemos $A=S T D T^{\dagger}$. Tomando $U \equiv S T$ e $V \equiv T^{\dagger}$, concluímos a demonstração.

Corolário (Decomposição de Valor Singular para uma Matriz Qualquer). Seja $A \in \operatorname{Mat}(\mathbb{C}, \mathrm{n} \times \mathrm{m})$ uma matriz retangular, $n \times m$ de coeficientes complexos, onde, sem perda de generalidade vamos supor que $n \geq m$. Existem matrizes unitárias $U \in \operatorname{Mat}(\mathbb{C}, n)$, $V \in \operatorname{Mat}(\mathbb{C}, m)$ e também uma matriz diagonal $D \in \operatorname{Mat}(\mathbb{R}, n \times m)$ com entradas não negativas de forma que

$$
A=U D V
$$

onde $\mathbb{I}_{n, m}$ é uma matriz retangular $n \times m$ que tem apenas uns na sua diagonal principal. Os elementos diagonais de $D$ são chamados valores singulares de $A$.

Demonstração. Dada a matriz $A \in \operatorname{Mat}(\mathbb{C}, \mathrm{n} \times \mathrm{m})$, definimos a matriz $A^{\prime}=A \mathbb{I}_{m, n}$, que obviamente é uma matriz quadrada $n \times n$. Aplicamos o corolário 3.2.1 a $A^{\prime}$ e obtemos:

$$
A^{\prime}=A \mathbb{I}_{m, n}=U D V \Rightarrow A=U D V \mathbb{I}_{n, m},
$$


onde $U, V, D \in \operatorname{Mat}(\mathbb{C}, n)$ e $U$ e $V$ são unitárias. Por outro lado, seja $\bar{V}=\mathbb{I}_{m, n} V \mathbb{I}_{n, m}$, onde $\bar{V}$ é uma matriz $m \times m$. A unitariedade de $\bar{V}$ se conclui usando a unitariedade de $V$. Dessa forma:

$$
V \mathbb{I}_{n, m}=\mathbb{I}_{n, m} \bar{V} \Rightarrow A=U D \mathbb{I}_{n, m} \bar{V}=U \tilde{D} \bar{V}
$$

onde $\tilde{D}=D \mathbb{I}_{n, m}$ é uma matriz $n \times m$, como queríamos demonstrar.

Teorema (Decomposição de Schmidt). Suponha que $|\psi\rangle$ seja um estado puro de um sistema composto de duas partes, $A$ e B. Existe uma base de estados ortonormais $\left|i_{A}\right\rangle$ para o subsistema $A$ e uma base de estados ortonormais $\left|i_{B}\right\rangle$ para o subsistema $B$ tais que

$$
|\psi\rangle=\sum_{i} \lambda_{i}\left|i_{A}\right\rangle \otimes\left|i_{B}\right\rangle
$$

onde os $\lambda_{i}$ são números reais não negativos que satisfazem $\sum_{i} \lambda_{i}^{2}=1$, conhecidos como coeficientes de Schmidt.

Demonstração. Seja $\mathcal{H}_{A}$ e $\mathcal{H}_{B}$ os espaços de estados associados, respectivamente, às partes $A$ e $B$ do sistema composto. Vamos considerar que $\operatorname{dim} \mathcal{H}_{A}=n$ e $\operatorname{dim} \mathcal{H}_{B}=m$, com $n \geq$ $m$, sem perda de generalidade. Sejam $\left\{\left|e_{A}^{i}\right\rangle\right\}$ e $\left\{\left|e_{B}^{j}\right\rangle\right\}$ bases para os espaços $\mathcal{H}_{A}$ e $\mathcal{H}_{B}$, respectivamente. Todo estado $|\psi\rangle \in \mathcal{H}=\mathcal{H}_{A} \otimes \mathcal{H}_{B}$ pode ser escrito como

$$
|\psi\rangle=\sum_{i=1}^{n} \sum_{j=1}^{m} a_{i j}\left|e_{A}^{i}\right\rangle \otimes\left|e_{B}^{j}\right\rangle
$$

onde $a_{i j}$ representa os coeficientes de uma certa matriz complexa $a, n \times m$. Pela corolário 3.2.1, podemos escrever $a=u d v$, com $u, n \times n$, e $v, m \times m$, sendo matrizes unitárias e $d$, $n \times m$, uma matriz diagonal com entradas não negativas, i.e. $d_{i j}=\lambda_{i} \delta_{i j}, \lambda_{i} \geq 0$. Em termos de componentes

$$
a_{i j}=\sum_{k=1}^{n} \sum_{l=1}^{m} u_{i k} d_{k l} v_{l j}=\sum_{k=1}^{n} \sum_{l=1}^{m} u_{i k} d_{k k} \delta_{k l} v_{l j}=\sum_{k=1}^{m} u_{i k} d_{k k} v_{k j}
$$

Reescrevendo o estado $|\psi\rangle$ :

$$
|\psi\rangle=\sum_{i=1}^{n} \sum_{j=1}^{m} \sum_{k=1}^{m} u_{i k} d_{k k} v_{k j}\left|e_{A}^{i}\right\rangle \otimes\left|e_{B}^{j}\right\rangle
$$


e definindo $\left|k_{A}\right\rangle=\sum_{i} u_{i k}\left|e_{A}^{i}\right\rangle,\left|k_{B}\right\rangle=\sum_{j} v_{k j}\left|e_{B}^{j}\right\rangle$ e $\lambda_{k}=d_{k k}$, obtemos:

$$
|\psi\rangle=\sum_{k=1}^{m} \lambda_{k}\left|k_{A}\right\rangle \otimes\left|k_{B}\right\rangle .
$$

As bases $\left|i_{A}\right\rangle$ e $\left|i_{B}\right\rangle$ apresentadas no teorema 3.2.1 são denominadas bases de Schmidt e a quantidade dos $\lambda_{i}$ que são não nulos é chamado de número de Schmidt para o estado $|\psi\rangle$. O número de Schmidt serve como ferramenta para quantificar a "quantidade" de emaranhamento de dado estado. Se o número de Schmidt for 1, então o estado em questão não é emaranhado. Exemplo. Para ilustrar o uso da decomposição de Schmidt como critério de separabilidade, considere um estado $|\psi\rangle \in \mathcal{H}=\mathcal{H}_{A} \otimes \mathcal{H}_{B}$, onde $\operatorname{dim} \mathcal{H}_{A}=2$ e $\operatorname{dim} \mathcal{H}_{B}=3$. Considere uma base $\{|0\rangle,|1\rangle\}$ para $\mathcal{H}_{A}$ e uma base $\{|0\rangle,|1\rangle,|2\rangle\}$ para $\mathcal{H}_{B}$ e

$$
|\psi\rangle=\frac{1}{\sqrt{3}}(|00\rangle+|01\rangle+|12\rangle)
$$

onde a notação $|i j\rangle$ é uma abreviação para $|i\rangle \otimes|j\rangle$. A matriz de coeficientes nessa base é:

$$
a=\frac{1}{\sqrt{3}}\left(\begin{array}{lll}
1 & 1 & 0 \\
0 & 0 & 1
\end{array}\right)
$$

Para escrevermos a decomposição de Schmidt de um certo estado com matriz de coeficientes $a$, devemos primeiramente encontrar os autovalores da matriz $a^{\dagger} a$. É possível mostrar que a matriz $a a^{\dagger}$ possui os mesmos autovalores não nulos que $a^{\dagger} a$, de forma que uma astúcia para calcular os coeficientes de Schmidt é escolher a matriz de menor tamanho entre $a a^{\dagger}$ e $a^{\dagger} a$. No nosso exemplo, a melhor opção é $a a^{\dagger}$ :

$$
a a^{\dagger}=\left(\begin{array}{cc}
2 / 3 & 0 \\
0 & 1 / 3
\end{array}\right)
$$

Este resultado nos diz que o número de Schmidt do estado $|\psi\rangle$ é 2, i.e. se trata de um estado emaranhado, e que os coeficientes de Schmidt são $\sqrt{2 / 3}$ e $\sqrt{1 / 3}$.

Uma outra maneira de decidir se um estado bipartido puro em dimensão finita é ou não emaranhado é usando o traço parcial.

Teorema. Seja $|\psi\rangle \in \mathcal{H}_{A} \otimes \mathcal{H}_{B}$, com $\mathcal{H}_{A}$ e $\mathcal{H}_{B}$ espaços de Hilbert de dimensão finita. $O$ estado $|\psi\rangle$ é emaranhado se e somente se as matrizes densidades associadas aos subsistemas A e $B$ representarem misturas, i.e. $\operatorname{tr}\left(\rho_{A}^{2}\right)<1$ e $\operatorname{tr}\left(\rho_{B}^{2}\right)<1$. 
Demonstração. Sejam $\left\{|i\rangle_{A}\right\}$ e $\left\{|j\rangle_{B}\right\}$ bases dos espaços $\mathcal{H}_{A}$ e $\mathcal{H}_{B}$, respectivamente. Podemos escrever um estado genérico $|\psi\rangle$ na forma:

$$
|\psi\rangle=\sum_{i, j} a_{i j}|i\rangle_{A} \otimes|j\rangle_{B}
$$

A matriz densidade associada a esse estado é:

$$
\begin{aligned}
\hat{\rho} & =|\psi\rangle\left\langle\psi \left|=\left(\sum_{i, j} a_{i j}|i\rangle_{A} \otimes|j\rangle_{B}\right)\left(\sum _ { k , l } a _ { k l } ^ { * } \left\langle\left.k\right|_{A} \otimes\left\langle\left. l\right|_{B}\right)\right.\right.\right.\right. \\
& =\sum_{i, j} \sum_{k, l}\left(a_{i j} a_{k l}^{*}\right)|i\rangle_{A}\left\langle\left. k\right|_{A} \otimes \mid j\right\rangle_{B}\left\langle\left. l\right|_{B}\right.
\end{aligned}
$$

Calculando a matriz densidade reduzida relativa ao sistema $A$, obtemos:

$$
\begin{aligned}
\hat{\rho}_{A} & =\operatorname{tr}_{B}(\hat{\rho})=\sum_{i, k}\left(\sum_{j} a_{i j} a_{k j}^{*}\right)|i\rangle_{A}\left\langle\left. k\right|_{A}\right. \\
& =\sum_{i, k}\left(a a^{\dagger}\right)_{i k}|i\rangle_{A}\left\langle\left. k\right|_{A},\right.
\end{aligned}
$$

o que nos diz que os elementos de matriz da matriz densidade reduzida $\hat{\rho}_{A}$ são precisamente os elementos de matriz da matriz $a a^{\dagger}$. Como sabemos, os autovalores da matriz $a a^{\dagger}$ são os coeficientes de Schmidt elevados ao quadrado. Seja $\left\{\lambda_{k}^{2}\right\}$ o conjunto de autovalores de $a a^{\dagger}$. É claro que

$$
\sum_{k} \lambda_{k}^{2}=1
$$

Observe que

$$
\operatorname{tr}\left(\hat{\rho}_{A}^{2}\right)=\sum_{k} \lambda_{k}^{4} \leq\left(\sum_{k} \lambda_{k}^{2}\right)^{2}=1,
$$

de fato, a igualdade é satisfeita apenas quando apenas um dos $\lambda_{k}$ é não nulo, i.e. quando $|\psi\rangle$ representa um estado não emaranhado.

Exemplo. Considere mais uma vez o estado do exemplo 3.2.1:

$$
|\psi\rangle=|\psi\rangle=\frac{1}{\sqrt{3}}(|00\rangle+|01\rangle+|12\rangle)
$$


A matriz densidade associada a esse estado é:

$$
\begin{aligned}
\hat{\rho}= & \frac{1}{3}(|00\rangle\langle 00|+| 01\rangle\langle 00|+| 12\rangle\langle 00|+| 00\rangle\langle 01|+| 01\rangle\langle 01| \\
& +|12\rangle\langle 01|+| 00\rangle\langle 12|+| 01\rangle\langle 12|+| 12\rangle\langle 12|) .
\end{aligned}
$$

Calculando a matriz densidade reduzida $\hat{\rho}_{A}$, temos:

$$
\hat{\rho}_{A}=\frac{1}{3}(2|0\rangle\langle 0|+| 1\rangle\langle 1|) \Rightarrow \hat{\rho}_{A}^{2}=\frac{1}{9}(4|0\rangle\langle 0|+| 1\rangle\langle 1|) .
$$

Portanto

$$
\operatorname{tr}\left(\hat{\rho}_{A}^{2}\right)=5 / 9<1
$$

i.e. $\hat{\rho}_{A}$ representa uma mistura e concluímos que $|\psi\rangle$ representa um estado emaranhado.

\subsection{Quantificação do Emaranhamento e a Entropia de von Neumann}

Atacaremos nessa seção o problema de dizer "quanto" emaranhamento um dado estado contém. Não existe até hoje uma medida de emaranhamento universal, i.e. uma noção que seja válida em todos os casos conhecidos, no entanto para o caso de sistemas puros bipartidos, foi mostrado por Bennett et al. [12] que a entropia de von Neumann é um bom medidor da quantidade de emaranhamento.

Definição. Seja $\rho$ a matriz densidade de um dado sistema quântico. Definimos a quantidade

$$
S(\rho)=-\operatorname{tr}\left(\rho \log _{2} \rho\right)
$$

que é chamada de entropia de von Neumann de $\rho$.

Definição. Seja $|\psi\rangle \in \mathcal{H}=\mathcal{H}_{A} \otimes \mathcal{H}_{B}$ e seja $\rho=|\psi\rangle\langle\psi|$. A quantidade de emaranhamento $E$ associado ao estado $|\psi\rangle$ é dada por

$$
E(\psi)=S\left(\rho_{A}\right)=S\left(\rho_{B}\right) \Leftrightarrow E(\psi)=-\operatorname{tr}\left(\rho_{A} \log _{2} \rho_{A}\right)=-\operatorname{tr}\left(\rho_{B} \log _{2} \rho_{B}\right)
$$

onde $\rho_{A}=\operatorname{tr}_{B}(\rho)$ e $\rho_{B}=\operatorname{tr}_{A}(\rho)$ são as matrizes densidade reduzidas associadas à matriz $\rho$.

Usando a decomposição de Schmidt de $|\psi\rangle$ podemos achar uma expressão para a quantidade de emaranhamento em termos apenas dos coeficientes de Schmidt. De fato, considere 
a decomposição de Schmidt de $|\psi\rangle$ :

$$
|\psi\rangle=\sum_{j} \lambda_{j}\left|j_{A}\right\rangle \otimes\left|j_{B}\right\rangle, \quad \lambda_{j} \in \mathbb{R}
$$

A matriz densidade $\rho$ associada a $|\psi\rangle$ é dada por

$$
\rho=|\psi\rangle\langle\psi|=\sum_{i, j} \lambda_{i} \lambda_{j}\left(\left|i_{A}\right\rangle\left\langle j_{A}\right|\right) \otimes\left(\left|i_{B}\right\rangle\left\langle j_{B}\right|\right)
$$

e as matrizes densidade reduzidas são

$$
\begin{aligned}
& \rho_{A}=\operatorname{tr}_{B}(\rho)=\sum_{j} \lambda_{j}^{2}\left|j_{A}\right\rangle\left\langle j_{A}\right|, \\
& \rho_{B}=\operatorname{tr}_{A}(\rho)=\sum_{j} \lambda_{j}^{2}\left|j_{B}\right\rangle\left\langle j_{B}\right| .
\end{aligned}
$$

Assim, a entropia de von Neumann associada ao estado $|\psi\rangle$ toma a forma:

$$
E(\psi)=-\sum_{j} \lambda_{j}^{2} \log _{2} \lambda_{j}^{2}
$$

Entropia de von Neumann tem a propriedade de se anular para estados separáveis * e de valer 1 para estados maximalmente emaranhados como $|\psi\rangle=\frac{1}{\sqrt{2}}(|01\rangle-|10\rangle)$.

Agora nós temos em mãos uma ferramenta, a entropia de von Neumann, que nos diz a quantidade de emaranhamento contida num estado puro bipartido, porém é natural se perguntar o que isso quer dizer, em outras palavras, dados dois estados $\left|\psi_{1}\right\rangle$ e $\left|\psi_{2}\right\rangle$ de forma que $E\left(\psi_{1}\right)>E\left(\psi_{2}\right)$, o que o estado $\left|\psi_{1}\right\rangle$ pode fazer que o estado $\left|\psi_{2}\right\rangle$ não pode?

Bennett et al. dão uma resposta a essa pergunta em [12]. Primeiramente eles definem uma unidade de emaranhamento, o ebit, dessa forma a entropia de von Neumann nos diz quantos ebits um dado estado $|\psi\rangle$ possui. Em [12] é mostrado que dado $n$ estados $|\psi\rangle$ com $E$ ebits de emaranhamento, é possível construir, usando operações locais e comunicação clássica, $m<n$ estados de singleto, i.e. $|s\rangle=\frac{1}{\sqrt{2}}(|01\rangle-|10\rangle)$. Além disso, foi mostrado que $m / n$ tende a $E$ quando $n \rightarrow \infty$.

A análise de Bennett et al. nos diz que quanto maior $E$, mais o estado é eficientemente transformado num singleto.

*o que é fácil de ver pois neste caso existe apenas um coeficiente de Schmidt e ele vale 1, o que nos diz que (3.3.1) se anula 


\subsection{Aplicação de Interesse}

Nesta seção vamos aplicar o critério de emaranhamento e calcular a entropia de von Neumann para o caso que nos interessará nesta dissertação, à saber

$$
|\psi\rangle=N \sum_{a, b=1}^{2} \alpha_{a b}|a\rangle \otimes|b\rangle, \quad N=\left(\sum_{a, b=1}^{2}\left|\alpha_{a b}\right|^{2}\right)^{-1 / 2}
$$

i.e. $|\psi\rangle \in \mathcal{H}_{A} \otimes \mathcal{H}_{B}$, onde $\operatorname{dim} \mathcal{H}_{A}=\operatorname{dim} \mathcal{H}_{B}=2$.

Calculando a matriz densidade associada ao estado definido pela equação (3.4.1), obtemos*

$$
\rho=|\psi\rangle\left\langle\psi\left|=N^{2} \sum_{a, b} \sum_{a^{\prime}, b^{\prime}} \alpha_{a b} \alpha_{a^{\prime} b^{\prime}}^{*}\right| a, b\right\rangle\left\langle a^{\prime}, b^{\prime}\right| .
$$

Tomando o traço parcial em relação ao subsistema $B$ calculamos a matriz densidade reduzida $\rho_{A}$ :

$$
\begin{aligned}
\rho_{A} & =\operatorname{tr}_{B}(\rho)=N^{2} \sum_{a, b} \sum_{a^{\prime}, b^{\prime}} \alpha_{a b} \alpha_{a^{\prime} b^{\prime}}^{*} \delta_{b b^{\prime}}|a\rangle\left\langle a^{\prime}\right| \\
& =N^{2} \sum_{a, b, c} \alpha_{a c} \alpha_{b c}^{*}|a\rangle\langle b| .
\end{aligned}
$$

Usando uma notação matricial, podemos reescrever a matriz densidade reduzida como:

$$
\rho_{A}=\boldsymbol{\alpha} \boldsymbol{\alpha}^{\dagger}
$$

onde $\boldsymbol{\alpha}$ é a matriz $2 \times 2$ cujos coeficientes são $\alpha_{a b}$. Como vimos, cada autovalor de $\rho_{A}$ corresponde ao quadrado de um coeficiente de Schmidt. O estado $|\psi\rangle$ representa um estado separável se, e somente se, possuir apenar um coeficiente de Schmidt, em outras palavras, se, e somente se, $\rho_{A}$ possuir apenas um autovalor não nulo. Como $\rho_{A}$ só apresenta dois autovalores, então uma condição necessária e suficiente para a separabilidade de $|\psi\rangle$ pode ser obtida do determinante de $\rho_{A}$, e consequentemente, do determinante de $\boldsymbol{\alpha}$.

Proposição. O estado $|\psi\rangle$ é separável se, e somente se, $\operatorname{det} \boldsymbol{\alpha}=0$.

Demonstração. A matriz densidade reduzida $\rho_{A}$ associada ao estado $|\psi\rangle$, na base de Schmidt, se escreve:

$$
\rho_{A}=\left(\begin{array}{cc}
\lambda_{1}^{2} & 0 \\
0 & \lambda_{2}^{2}
\end{array}\right)
$$

${ }^{*}$ Note que $|a, b\rangle \equiv|a\rangle \otimes|b\rangle$. 
onde $\lambda_{1}$ e $\lambda_{2}$ são os coeficientes de Schmidt de $|\psi\rangle$. Admitindo que $|\psi\rangle$ não é o vetor nulo, então

$$
\operatorname{det} \rho_{A}=\lambda_{1}^{2} \lambda_{2}^{2}=0 \Rightarrow \lambda_{1}=0 \quad \text { ou } \quad \lambda_{2}=0
$$

Em ambos os casos, $\rho_{A}$ representa um estado puro, e logo, $|\psi\rangle$ é um estado separável. Reciprocamente, se $|\psi\rangle$ é um estado separável, então admite apenas um coeficiente de Schmidt não nulo. Dessa forma, concluímos que $\operatorname{det} \rho_{A}=0$. Observe que

$$
\operatorname{det} \rho_{A}=\operatorname{det}\left(\boldsymbol{\alpha} \boldsymbol{\alpha}^{\dagger}\right)=|\operatorname{det} \boldsymbol{\alpha}|^{2}=0 \Leftrightarrow \operatorname{det} \boldsymbol{\alpha}=0
$$

Com isso mostramos que $\operatorname{det} \boldsymbol{\alpha}=0$ é uma condição necessária e suficiente para a separabilidade de $|\psi\rangle$.

Agora vamos calcular a quantidade de emaranhamento $E$ contido no estado $|\psi\rangle$, supondo que det $\boldsymbol{\alpha} \neq 0$. Como vimos, precisamos apenas dos coeficientes de Schmidt de $|\psi\rangle$ para calcular $E$ (vide equação (3.3.1)). Primeiramente vamos calcular os autovalores da matriz densidade reduzida $\rho_{A}$. Como $\rho_{A}$ é uma matriz $2 \times 2$, então seus autovalores são as soluções da equação

$$
\xi^{2}-\left(\operatorname{tr}\left(\rho_{A}\right)\right) \xi+\operatorname{det} \rho_{A}=0
$$

Lembrando que $\operatorname{tr}\left(\rho_{A}\right)=1$, pois $\rho_{A}$ é uma matriz densidade, temos:

$$
\xi_{ \pm}=\frac{1}{2}\left(1 \pm \sqrt{1-4 \operatorname{det} \rho_{A}}\right)
$$

Claro que $\xi_{ \pm}=\lambda_{ \pm}^{2}$, onde os $\lambda_{ \pm}$são os coeficientes de Schmidt de $|\psi\rangle$, e $\operatorname{det} \rho_{A}=|\operatorname{det} \boldsymbol{\alpha}|^{2}$. A quantidade de emaranhamento é dada por:

$$
\begin{aligned}
E(\psi) & =-\lambda_{+}^{2} \log _{2} \lambda_{+}^{2}-\lambda_{-}^{2} \log _{2} \lambda_{-}^{2} \\
& =-\frac{1}{2}\left[\left(1+\sqrt{1-4|\operatorname{det} \boldsymbol{\alpha}|^{2}}\right) \log _{2} \lambda_{+}^{2}+\left(1-\sqrt{1-4|\operatorname{det} \boldsymbol{\alpha}|^{2}}\right) \log _{2} \lambda_{-}^{2}\right] \\
& =-\frac{1}{2}\left[\log _{2}\left(\lambda_{+}^{2} \lambda_{-}^{2}\right)+\sqrt{1-4|\operatorname{det} \boldsymbol{\alpha}|^{2}} \log _{2} \frac{\lambda_{+}^{2}}{\lambda_{-}^{2}}\right] \\
& =-\left[\log _{2} d+\sqrt{1-4|\operatorname{det} \boldsymbol{\alpha}|^{2}} \log _{2}\left(\frac{1+\sqrt{1-4|\operatorname{det} \boldsymbol{\alpha}|^{2}}}{2|\operatorname{det} \boldsymbol{\alpha}|}\right)\right]
\end{aligned}
$$




$$
\begin{gathered}
\therefore E(\psi)=-\left[\log _{2}|\operatorname{det} \boldsymbol{\alpha}|+\sqrt{1-4|\operatorname{det} \boldsymbol{\alpha}|^{2}} \log _{2}\left(\frac{1+\sqrt{1-4|\operatorname{det} \boldsymbol{\alpha}|^{2}}}{2|\operatorname{det} \boldsymbol{\alpha}|}\right)\right], \\
0 \leq|\operatorname{det} \boldsymbol{\alpha}| \leq \frac{1}{2}
\end{gathered}
$$

\subsection{Estados Puros Bipartidos em Dimensão Infinita: Cri- tério de Peres-Horodecki-Simon (PHS)}

No artigo [24], Simon apresenta um critério de separabilidade necessário e suficiente para estados gaussianos bipartidos em dimensão infinita. Tal método é baseado no critério de positividade da transposição parcial da matriz densidade, comumente chamado de critério PPT (positive partial transpose), apresentada por Peres em [20] e posteriormente refinado por Horodecki em [17]. Em [20], Peres mostra que o critério PPT fornece uma condição suficiente para separabilidade de um estado $\hat{\rho}$. Em [17] é mostrado que para sistemas de baixa dimensionalidade, a saber $2 \times 2$ e $2 \times 3$, o critério PPT é uma condição necessária e suficiente para separabilidade.

Seja um sistema bipartido de dois modos, descrito pelos operadores $\hat{a}_{k}=\left(\hat{q}_{k}+i \hat{p}_{k}\right) / \sqrt{2}$, $k=1,2$. O modo 1 corresponde ao sistema $A$ e o modo 2 corresponde ao sistema $B$. Podemos expressar as relações de comutação entre $\hat{q}_{k}$ e $\hat{p}_{k}$ da seguinte forma:

$$
\hat{\xi}=\left(\hat{q}_{1}, \hat{p}_{1}, \hat{q}_{2}, \hat{p}_{2}\right)^{t}
$$

As relações de comutação são dadas por:

$$
\left[\hat{\xi}_{\alpha}, \hat{\xi}_{\beta}\right]=i \Omega_{\alpha \beta}, \quad \alpha, \beta=1,2,3,4
$$

e a matriz $\Omega$ é dada por:

$$
\Omega=\left(\begin{array}{ll}
J & 0 \\
0 & J
\end{array}\right), \quad J=\left(\begin{array}{cc}
0 & 1 \\
-1 & 0
\end{array}\right)
$$

Seguindo o que fez Simon em [24], nosso objetivo é primeiramente mostrar o que significa uma transposição parcial no formalismo da distribuição de Wigner e em seguida, usando como ferramenta tal formalismo, reformular o resultado de Peres. Seja $\hat{\rho}$ a matriz densidade 
correspondente a um estado puro separável $|\psi\rangle=\left|\phi^{A}\right\rangle \otimes\left|\phi^{B}\right\rangle \in \mathcal{H}=\mathcal{H}_{A} \otimes \mathcal{H}_{B}{ }^{*}$

$$
\begin{aligned}
\hat{\rho} & =|\psi\rangle\left\langle\psi \left|=\left(\left|\phi^{A}\right\rangle \otimes\left|\phi^{B}\right\rangle\right)\left(\left\langle\phi^{A}\right| \otimes\left\langle\phi^{B}\right|\right)\right.\right. \\
& =\left|\phi^{A}\right\rangle\left\langle\phi^{A}|\otimes| \phi^{B}\right\rangle\left\langle\phi^{B}\right| \equiv \hat{\rho}_{A} \otimes \hat{\rho}_{B} .
\end{aligned}
$$

Definimos a transposição parcial como sendo uma transposição em relação a um subsistema, em particular, a transposição parcial de $\hat{\rho}$ em relação ao subsistema $B$ é a matriz densidade $\hat{\rho}^{t_{B}} \equiv \hat{\rho}_{A} \otimes \hat{\rho}_{B}^{t}$. De maneira análoga definimos a transposição parcial em relação ao subsistema A.

Dada a matriz $\hat{\rho}$, podemos escrever sua distribuição de Wigner:

$$
W(\boldsymbol{q}, \boldsymbol{p})=\frac{1}{\pi^{2}} \int d^{2} q^{\prime} e^{2 i \boldsymbol{p} \cdot \boldsymbol{q}^{\prime}}\left\langle\boldsymbol{q}-\boldsymbol{q}^{\prime}|\hat{\rho}| \boldsymbol{q}+\boldsymbol{q}^{\prime}\right\rangle
$$

onde $\boldsymbol{q}=\left(q_{1}, q_{2}\right)$ e $\boldsymbol{p}=\left(p_{1}, p_{2}\right)$.

Teorema. Seja $\hat{\rho}$ um estado puro separável, i.e. $\hat{\rho}=\hat{\rho}_{A} \otimes \hat{\rho}_{B}$, cuja distribuição de Wigner é $W(\xi)$. Uma transposição parcial em relação ao subsistema $B$ associa a $\hat{\rho}$ uma nova matriz densidade $\hat{\sigma}$ cuja distribuição de Wigner $W^{\prime}(\xi)=W(\Lambda \xi)$, onde $\xi=\left(q_{1}, p_{1}, q_{2}, p_{2}\right)$ e $\Lambda=$ $\operatorname{diag}(1,1,1,-1)$.

Demonstração. Escrevendo explicitamente a distribuição de Wigner para $\hat{\rho}=\hat{\rho}_{A} \otimes \hat{\rho}_{B}$, obtemos:

$$
\begin{aligned}
W(\boldsymbol{q}, \boldsymbol{p}) & =\frac{1}{\pi^{2}} \int d^{2} q^{\prime} e^{2 i \boldsymbol{p} \cdot \boldsymbol{q}^{\prime}}\left\langle\boldsymbol{q}-\boldsymbol{q}^{\prime}|\hat{\rho}| \boldsymbol{q}+\boldsymbol{q}^{\prime}\right\rangle \\
& =\frac{1}{\pi^{2}} \int d^{2} q^{\prime} e^{2 i \boldsymbol{p} \cdot \boldsymbol{q}^{\prime}}\left\langle\boldsymbol{q}-\boldsymbol{q}^{\prime}\left|\hat{\rho}_{A} \otimes \hat{\rho}_{B}\right| \boldsymbol{q}+\boldsymbol{q}^{\prime}\right\rangle \\
W(\boldsymbol{q}, \boldsymbol{p}) & =\frac{1}{\pi^{2}} \int d q_{1}^{\prime} d q_{2}^{\prime} e^{2 i\left(p_{1} q_{1}^{\prime}+p_{2} q_{2}^{\prime}\right)}\left\langle q_{1}-q_{1}^{\prime}\left|\hat{\rho}_{A}\right| q_{1}+q_{1}^{\prime}\right\rangle\left\langle q_{2}-q_{2}^{\prime}\left|\hat{\rho}_{B}\right| q_{2}+q_{2}^{\prime}\right\rangle .
\end{aligned}
$$

A matriz densidade $\hat{\sigma}=\hat{\rho}^{t_{B}}=\hat{\rho}_{A} \otimes \hat{\rho}_{B}^{t}$ tem como distribuição de Wigner:

$$
\begin{aligned}
W^{\prime}(\boldsymbol{q}, \boldsymbol{p}) & =\frac{1}{\pi^{2}} \int d^{2} q^{\prime} e^{2 i \boldsymbol{p} \cdot \boldsymbol{q}^{\prime}}\left\langle\boldsymbol{q}-\boldsymbol{q}^{\prime}|\hat{\sigma}| \boldsymbol{q}+\boldsymbol{q}^{\prime}\right\rangle \\
& =\frac{1}{\pi^{2}} \int d^{2} q^{\prime} e^{2 i \boldsymbol{p} \cdot \boldsymbol{q}^{\prime}}\left\langle\boldsymbol{q}-\boldsymbol{q}^{\prime}\left|\hat{\rho}_{A} \otimes \hat{\rho}_{B}^{t}\right| \boldsymbol{q}+\boldsymbol{q}^{\prime}\right\rangle \\
W^{\prime}(\boldsymbol{q}, \boldsymbol{p}) & =\frac{1}{\pi^{2}} \int d q_{1}^{\prime} d q_{2}^{\prime} e^{2 i\left(p_{1} q_{1}^{\prime}+p_{2} q_{2}^{\prime}\right)}\left\langle q_{1}-q_{1}^{\prime}\left|\hat{\rho}_{A}\right| q_{1}+q_{1}^{\prime}\right\rangle\left\langle q_{2}+q_{2}^{\prime}\left|\hat{\rho}_{B}\right| q_{2}-q_{2}^{\prime}\right\rangle
\end{aligned}
$$

${ }^{*} \mathrm{O}$ caso de estados mistos não nos interessa nesse trabalho, mas o resultado pode ser mostrado para misturas em geral. 
Fazendo a mudança de variável $q_{2}^{\prime} \rightarrow-q_{2}^{\prime}$ temos:

$$
\begin{aligned}
W^{\prime}\left(q_{1}, p_{1}, q_{2}, p_{2}\right) & =\frac{1}{\pi^{2}} \int d q_{1}^{\prime} d q_{2}^{\prime} e^{2 i\left(p_{1} q_{1}^{\prime}+\left(-p_{2}\right) q_{2}^{\prime}\right)}\left\langle q_{1}-q_{1}^{\prime}\left|\hat{\rho}_{A}\right| q_{1}+q_{1}^{\prime}\right\rangle\left\langle q_{2}-q_{2}^{\prime}\left|\hat{\rho}_{B}\right| q_{2}+q_{2}^{\prime}\right\rangle \\
W^{\prime}\left(q_{1}, p_{1}, q_{2}, p_{2}\right) & =W\left(q_{1}, p_{1}, q_{2},-p_{2}\right)
\end{aligned}
$$

Usando a notação $\xi=\left(q_{1}, p_{1}, q_{2}, p_{2}\right)$ e $\Lambda=\operatorname{diag}(1,1,1,-1)$, vemos que a transposição parcial em relação ao subsistema $B$ corresponde a seguinte transformação na função de Wigner:

$$
W(\xi) \rightarrow W(\Lambda \xi)
$$

o que corresponde a uma reflexão da coordenada de momento associada ao subsistema em questão. O mesmo resultado pode ser obtido para transposição parcial em relação ao subsistema $A$.

No que segue, apresentaremos um resultado presente em [25] no qual as relações de incerteza de Heisenberg são escritas usando a matriz de correlações. Estamos interessados em calcular o valor esperado de produtos do tipo $\Delta \hat{\xi}_{\alpha} \Delta \hat{\xi}_{\beta}$, onde $\Delta \hat{\xi}_{\alpha}=\hat{\xi}_{\alpha}-\left\langle\hat{\xi}_{\alpha}\right\rangle,\left\langle\hat{\xi}_{\alpha}\right\rangle=$ $\operatorname{tr}\left(\hat{\xi}_{\alpha} \hat{\rho}\right), \alpha=1,2,3,4$ e $\hat{\rho}$ representa o estado do sistema. No tocante à estatística, podemos também trabalhar com o formalismo de Wigner, onde teremos $\Delta \xi_{\alpha}=\xi_{\alpha}-\left\langle\xi_{\alpha}\right\rangle$ e $\left\langle\xi_{\alpha}\right\rangle=$ $\int d^{4} \xi \xi_{\alpha} W(\xi)$. Como a distribuição de Wigner fornece as mesmas previsões estatísticas que $\hat{\rho}$, então $\left\langle\hat{\xi}_{\alpha}\right\rangle=\left\langle\xi_{\alpha}\right\rangle$. Observe que:

$$
\begin{aligned}
\Delta \hat{\xi}_{\alpha} \Delta \hat{\xi}_{\beta} & =\frac{1}{2}\left(\left\{\Delta \hat{\xi}_{\alpha}, \Delta \hat{\xi}_{\beta}\right\}+\left[\Delta \hat{\xi}_{\alpha}, \Delta \hat{\xi}_{\beta}\right]\right) \\
& =\frac{1}{2}\left(\left\{\Delta \hat{\xi}_{\alpha}, \Delta \hat{\xi}_{\beta}\right\}+i \Omega_{\alpha \beta}\right)
\end{aligned}
$$

de forma que

$$
\left\langle\Delta \hat{\xi}_{\alpha} \Delta \hat{\xi}_{\beta}\right\rangle=\left\langle\frac{1}{2}\left\{\Delta \hat{\xi}_{\alpha}, \Delta \hat{\xi}_{\beta}\right\}\right\rangle+\frac{i}{2} \Omega_{\alpha \beta}
$$

Denominamos a matriz $V$ cujos coeficientes são $V_{\alpha \beta}=\left\langle\frac{1}{2}\left\{\Delta \hat{\xi}_{\alpha}, \Delta \hat{\xi}_{\beta}\right\}\right\rangle$ de matriz de correlações. Esses elementos de matriz podem ser calculados de duas formas distintas, a saber:

$$
V_{\alpha \beta}=\left\langle\frac{1}{2}\left\{\Delta \hat{\xi}_{\alpha}, \Delta \hat{\xi}_{\beta}\right\}\right\rangle=\left\{\begin{array}{l}
\frac{1}{2} \operatorname{tr}\left(\left\{\Delta \hat{\xi}_{\alpha}, \Delta \hat{\xi}_{\beta}\right\} \hat{\rho}\right) \\
\int d^{4} \xi \Delta \xi_{\alpha} \Delta \xi_{\beta} W(\xi)
\end{array}\right.
$$

Em [25] é demonstrado o seguinte resultado: 
Teorema. Se $\hat{\rho}$ é um estado contínuo bipartido qualquer então,

$$
V_{\alpha \beta}+\frac{i}{2} \Omega_{\alpha \beta} \geq 0, \quad \forall \alpha, \beta
$$

Sabemos pelo resultado de Peres em [20] que uma condição necessária para separabilidade de um estado $\hat{\rho}$ é que sua transposição parcial seja ainda uma matriz densidade. Em termos de distribuições de Wigner temos então que uma condição necessária para separabilidade é que dada uma distribuição de Wigner $W(\xi)$ associada ao estado $\hat{\rho}$, a função $W(\Lambda \xi)$, onde $\Lambda=\operatorname{diag}(1,1,1,-1)$, também deve ser uma função de Wigner, em outras palavras ela deve satisfazer desigualdades como as apresentadas no teorema acima. Apresentemos detalhadamente quais desigualdes $W(\Lambda \xi)$ deve satisfazer. Seja

$$
V_{\alpha \beta}=\int d^{4} \xi \Delta \xi_{\alpha} \Delta \xi_{\beta} W(\xi)
$$

a matriz de correlações associada a $W(\xi)$. A matriz de correlações associada a $W(\Lambda \xi)$ é dada por:

$$
\tilde{V}_{\alpha \beta}=\int d^{4} \xi \Delta \xi_{\alpha} \Delta \xi_{\beta} W(\Lambda \xi)
$$

fazendo a transformação $\xi \rightarrow \Lambda \xi$, temos:

$$
\begin{aligned}
\tilde{V}_{\alpha \beta} & =\int d^{4} \xi\left(\sum_{\mu=1}^{4} \Lambda_{\alpha \mu} \Delta \xi_{\mu}\right)\left(\sum_{\nu=1}^{4} \Lambda_{\beta \nu} \Delta \xi_{\nu}\right) W(\xi) \\
& =\sum_{\mu, \nu} \Lambda_{\alpha \mu} \Lambda_{\beta \nu} \int d^{4} \xi \Delta \xi_{\mu} \Delta \xi_{\nu} W(\xi) \\
\tilde{V}_{\alpha \beta} & =\sum_{\mu, \nu} \Lambda_{\alpha \mu} \Lambda_{\beta \nu} V_{\mu \nu}
\end{aligned}
$$

Dessa forma, temos que:

$$
\tilde{V}=\Lambda V \Lambda
$$

Para que $W(\Lambda \xi)$ seja uma distribuição de Wigner legítima, é necessário que:

$$
\tilde{V}+\frac{i}{2} \Omega \geq 0 \Leftrightarrow \Lambda V \Lambda+\frac{i}{2} \Omega \geq 0 \Leftrightarrow V+\frac{i}{2} \tilde{\Omega} \geq 0
$$

onde $\tilde{\Omega}=\Lambda \Omega \Lambda=\left(\begin{array}{cc}J & 0 \\ 0 & -J\end{array}\right)$ e usamos o fato de que $\Lambda^{2}=\mathbb{I}$. Assim, uma condição 
necessária para separabilidade de um estado $\hat{\rho}$ é que a desigualdade

$$
V+\frac{i}{2} \tilde{\Omega} \geq 0
$$

seja satisfeita. Esse critério é também operacional, pois, dado $\hat{\rho}$, podemos calcular sua matriz de correlação $V$. Após diagonalizar a matriz $V+\frac{i}{2} \tilde{\Omega}$, se obtivermos algum autovalor negativo, então o estado é emaranhado.

Simon em [24] foi além e provou o seguinte resultado:

Teorema. Um sistema gaussiano bipartido é separável se, e somente se, sua matriz de correlações satisfaz a seguinte desigualdade:

$$
\operatorname{det} A \operatorname{det} B+\left(\frac{1}{4}-|\operatorname{det} C|\right)^{2}-\operatorname{tr}\left(A J C J B J C^{t} J\right)-\frac{1}{4}(\operatorname{det} A+\operatorname{det} B) \geq 0
$$

onde

$$
V=\left(\begin{array}{ll}
A & C \\
C^{t} & B
\end{array}\right)
$$

$e$

$$
J=\left(\begin{array}{cc}
0 & 1 \\
-1 & 0
\end{array}\right)
$$

Um resultado que é útil para se demonstrar o teorema acima é o seguinte:

Lema. Se $\hat{\rho}$ é um estado gaussiano bipartido e $\operatorname{det} C \geq 0$, então ele é separável. A matriz $C$ é retirada da matriz de correlações $V$,

$$
V=\left(\begin{array}{ll}
A & C \\
C^{t} & B
\end{array}\right)
$$




\section{Capítulo 4}

\section{Emaranhamento de Quasifótons}

Neste capítulo vamos aplicar os métodos apresentados no capítulo 3 para discutir propriedades de emaranhamento dos estados de número definido de quasifótons apresentados no capítulo 2. Primeiramente abordaremos o problema desprezando contribuições de ordem superior na constante de estrutura fina $\alpha$ no vácuo de quasifótons, de forma que o mesmo se torna igual ao vácuo de fótons. Consideramos na seção 3 estados de dois quasifótons de frequências distintas e analisamos a questão do emaranhamento dessas frequências na ausência de campo externo e analisamos o emaranhamento entre polarizações no caso onde temos um campo magnético constante na direção de propagação dos fótons. Em seguida, nos restringimos ao caso monocromático e estudamos exatamente as propriedades de emaranhamento do vácuo de quasifótons. Partindo do vácuo exato, estudamos a questão do emaranhamento para estados de dois quasifótons, negligenciando amplitudes com número total de fótons diferente do número total de quasifótons.

\subsection{Emaranhamento com Vácuo de Quasifótons Aproxi- mado}

Comçaremos nossa análise considerando que o vácuo de quasifótons é igual ao vácuo de fótons, em outros termos, vamos considerar que o vácuo da teoria interagente é igual ao vácuo da teoria livre. Tal consideração é frequentemente feita em eletrodinâmica quântica quando fazemos análises perturbativas e é uma boa aproxiamção no regime de baixas energias, onde a constante de acoplamento da teoria é pequena. Trataremos o caso dicromático nessa seção. Para cada fóton temos informação sobre sua polarização e sua frequência. Sempre usaremos uma das informações para diferenciar fótons e olharemos emaranhamento na outra. 


\subsubsection{Estados de Dois Quasifótons com Vácuo de Quasifótons Apro- ximado}

Considere os operadores $c_{j, \lambda}^{\dagger}$ e $c_{j, \lambda}$ de criação e aniquilação de quasifótons introduzidos no capítulo 2 na ausência de campo externo. O índice $j$ diz respeito à frequência $r_{j}$ do quasifóton e o índice $\lambda$ à polarização. Vamos considerar o caso de polarização linear e dicromático, ou seja, o índice $j$ tem duas possibilidades, digamos $j=1,2^{*}$. Sejam $a_{i, \lambda}^{\dagger}$ e $a_{i, \lambda}$ os operadores de criação e aniquilação de fótons, onde o índice $i$ diz respeito à frequência $\omega_{i}$ do fóton e o índice $\lambda$ à polarização. Podemos expressar os operadores de criação e aniquilação dos quasifótons em termos dos operadores $a_{i, \lambda}^{\dagger}$ e $a_{i, \lambda}$ :

$$
c_{j, \lambda}=\sum_{i}\left(a_{i, \lambda} \phi_{i j}-a_{i, \lambda}^{\dagger} \psi_{i j}\right) \quad \text { e } \quad c_{j, \lambda}^{\dagger}=\sum_{i}\left(a_{i, \lambda}^{\dagger} \phi_{i j}-a_{i, \lambda} \psi_{i j}\right),
$$

onde os coeficientes $\phi_{i j}$ e $\psi_{i j}$ dependem do momento $p$ dos elétrons, da densidade $\rho$ de elétrons, das frequências $\omega_{1}$ e $\omega_{2}$ dos fótons e da constante de estrutura fina $\alpha$. Explicitamente, temos:

$$
\begin{aligned}
\phi_{i j} & =\frac{1}{2}\left[\left(\frac{\omega_{i}}{r_{j}}\right)^{1 / 2}+\left(\frac{r_{j}}{\omega_{i}}\right)^{1 / 2}\right] q_{i j}, \\
\psi_{i j} & =\frac{1}{2}\left[\left(\frac{\omega_{i}}{r_{j}}\right)^{1 / 2}-\left(\frac{r_{j}}{\omega_{i}}\right)^{1 / 2}\right] q_{i j}, \\
q_{i j} & =\frac{1}{\left(r_{j}^{2}-\omega_{i}^{2}\right)}\left[\sum_{l}\left(r_{j}^{2}-\omega_{l}^{2}\right)^{-2}\right]^{-1 / 2}, \\
r_{1} & =\left[\frac{1}{2}\left(\omega_{1}^{2}+\omega_{2}^{2}+\alpha \frac{\rho}{n_{\mu} p^{\mu}}-\sqrt{\left(\omega_{2}^{2}-\omega_{1}^{2}\right)+\left(\alpha \frac{\rho}{n_{\mu} p^{\mu}}\right)^{2}}\right)\right]^{1 / 2}, \\
r_{2} & =\left[\frac{1}{2}\left(\omega_{1}^{2}+\omega_{2}^{2}+\alpha \frac{\rho}{n_{\mu} p^{\mu}}+\sqrt{\left(\omega_{2}^{2}-\omega_{1}^{2}\right)+\left(\alpha \frac{\rho}{n_{\mu} p^{\mu}}\right)^{2}}\right)\right]^{1 / 2} .
\end{aligned}
$$

Note que na expressão (4.1.1) não está presente o termo linear da transformação canônica, i.e. $\gamma_{j, \lambda}$, que tínhamos na expressão (2.2.32). Tal termo pode ser tomado a zero fazendo uma escolha conveniente de referencial, visto que ele é nulo caso o momento do elétron seja nulo

${ }^{*}$ Note que nas soluções apresentadas no capítulo 2 não tínhamos a liberdade de escolher quais frequências ou que número de frequências usar, todas as frequências do espectro eletromagnético estavam presentes. De fato, temos tantas frequências distintas para quasifótons quanto temos para fótons, de forma que deveríamos levar em conta uma infinidade de frequências. Nos restringimos ao caso de duas frequências tendo em mente que condições de contorno devidamente escolhidas sejam capazes de tornar dois modos do campo eletromagnético bem mais importantes que os outros. 
ou paralelo à direção de propagação dos fótons, como pode ser visto na equação (2.2.27).

Seja $\left|r_{j}, \lambda ; r_{j^{\prime}}, \lambda^{\prime}\right\rangle$ o estado composto de dois quasifótons de frequências $r_{j}$ e $r_{j^{\prime}}$ e polarizações $\lambda$ e $\lambda^{\prime}$, i.e.:

$$
\left|r_{j}, \lambda ; r_{j^{\prime}}, \lambda^{\prime}\right\rangle=N c_{j, \lambda}^{\dagger} c_{j^{\prime}, \lambda^{\prime}}^{\dagger}|\Omega\rangle
$$

onde $N$ é uma constante de normalização e $|\Omega\rangle$ é o vácuo de quasifótons, i.e. o estado que satisfaz:

$$
c_{j, \lambda}|\Omega\rangle=0, \quad \forall j, \lambda .
$$

Podemos reescrever a (4.1.8) usando a expressão de $c_{j, \lambda}$ apresentada em (4.1.1):

$$
\left(a_{i, \lambda} \phi_{i j}-a_{i, \lambda}^{\dagger} \psi_{i j}\right)|\Omega\rangle=0
$$

Claramente a solução de (4.1.9) não é o vácuo de fótons $|0 ; 0\rangle$, porém vamos considerar a aproximação em que $|\Omega\rangle \approx|0 ; 0\rangle$. Escrevendo explicitamente o estado (4.1.7) nessa aproximação, temos:

$$
\begin{aligned}
\left|r_{j}, \lambda ; r_{j^{\prime}}, \lambda^{\prime}\right\rangle & =N \sum_{i, i^{\prime}}\left(a_{i, \lambda}^{\dagger} \phi_{i j}-a_{i, \lambda} \psi_{i j}\right)\left(a_{i^{\prime}, \lambda^{\prime}}^{\dagger} \phi_{i^{\prime} j^{\prime}}-a_{i^{\prime}, \lambda^{\prime}} \psi_{i^{\prime} j^{\prime}}\right)|0 ; 0\rangle \\
& =N \sum_{i, i^{\prime}}\left(\phi_{i j} \phi_{i^{\prime} j^{\prime}} a_{i, \lambda}^{\dagger} a_{i^{\prime}, \lambda^{\prime}}^{\dagger}+\psi_{i j} \psi_{i^{\prime} j^{\prime}} a_{i, \lambda} a_{i^{\prime}, \lambda^{\prime}}-\phi_{i j} \psi_{i^{\prime} j^{\prime}} a_{i, \lambda}^{\dagger} a_{i^{\prime}, \lambda^{\prime}}-\psi_{i j} \phi_{i^{\prime} j^{\prime}} a_{i, \lambda} a_{i^{\prime}, \lambda^{\prime}}^{\dagger}\right)|0 ; 0\rangle \\
\left|r_{j}, \lambda ; r_{j^{\prime}}, \lambda^{\prime}\right\rangle & =N \sum_{i, i^{\prime}}\left(\phi_{i j} \phi_{i^{\prime} j^{\prime}}\left|\omega_{i}, \lambda ; \omega_{i^{\prime}}, \lambda^{\prime}\right\rangle-\psi_{i j} \phi_{i^{\prime} j^{\prime}} \delta_{i i^{\prime}} \delta_{\lambda \lambda^{\prime}}|0 ; 0\rangle\right)
\end{aligned}
$$

Considerando o caso de quasifótons de polarizações distintas, obtemos:

$$
\left|r_{j}, \lambda ; r_{j^{\prime}}, \lambda^{\prime}\right\rangle=N \sum_{i, i^{\prime}} \phi_{i j} \phi_{i^{\prime} j^{\prime}}\left|\omega_{i}, \lambda ; \omega_{i^{\prime}}, \lambda^{\prime}\right\rangle, \quad N=\left[\sum_{i, i^{\prime}}\left(\phi_{i j} \phi_{i^{\prime} j^{\prime}}\right)^{2}\right]^{-1 / 2}
$$

Queremos saber quando $\left|r_{j}, \lambda ; r_{j^{\prime}}, \lambda^{\prime}\right\rangle$ representa um estado emaranhado. Como vimos no capítulo (3), podemos obter essa informação analisando quando os subsistemas do sistema descrito pelo estado $\left|r_{j}, \lambda ; r_{j^{\prime}}, \lambda^{\prime}\right\rangle$ não são estados puros. A matriz densidade associada a este estado é:

$$
\begin{aligned}
\hat{\rho}\left(r_{j}, r_{j^{\prime}}\right) & =\left|r_{j}, \lambda ; r_{j^{\prime}}, \lambda^{\prime}\right\rangle\left\langle r_{j}, \lambda ; r_{j^{\prime}}, \lambda^{\prime}\right| \\
& =N^{2} \sum_{i_{1}, i_{1}^{\prime}} \sum_{i_{2}, i_{2}^{\prime}} \phi_{i_{1} j} \phi_{i_{1}^{\prime} j^{\prime}} \phi_{i_{2} j} \phi_{i_{2}^{\prime} j^{\prime}}\left|\omega_{i_{1}}, \lambda ; \omega_{i_{1}^{\prime}}, \lambda^{\prime}\right\rangle\left\langle\omega_{i_{2}}, \lambda ; \omega_{i_{2}^{\prime}}, \lambda^{\prime}\right|
\end{aligned}
$$


Tomando o traço parcial de $\hat{\rho}$ em relação ao subsistema com polarização $\lambda^{\prime}$, chegamos em:

$$
\begin{aligned}
\hat{\rho}_{\lambda}\left(r_{j}, r_{j^{\prime}}\right) & =\operatorname{tr}_{\lambda^{\prime}}\left(\hat{\rho}\left(r_{j}, r_{j^{\prime}}\right)\right)=N^{2} \sum_{i_{1}, i_{1}^{\prime}} \sum_{i_{2}, i_{2}^{\prime}} \phi_{i_{1} j} \phi_{i_{1}^{\prime} j^{\prime}} \phi_{i_{2} j} \phi_{i_{2}^{\prime} j^{\prime}} \delta_{i_{1}^{\prime} i_{2}^{\prime}}\left|\omega_{i_{1}}, \lambda\right\rangle\left\langle\omega_{i_{2}}, \lambda\right| \\
& =N^{2} \sum_{i_{1}, i_{2}, i_{1}^{\prime}} \phi_{i_{1} j} \phi_{i_{1}^{\prime} j^{\prime}} \phi_{i_{2} j} \phi_{i_{1}^{\prime} j^{\prime}}\left|\omega_{i_{1}}, \lambda\right\rangle\left\langle\omega_{i_{2}}, \lambda\right|
\end{aligned}
$$

Para simplificar a notação, faremos $i_{1} \rightarrow k, i_{2} \rightarrow l$ e $i_{1}^{\prime} \rightarrow m:$

$$
\hat{\rho}_{\lambda}\left(r_{j}, r_{j^{\prime}}\right)=N^{2} \sum_{k, l, m} \phi_{k j} \phi_{m j^{\prime}} \phi_{l j} \phi_{m j^{\prime}}\left|\omega_{k}, \lambda\right\rangle\left\langle\omega_{l}, \lambda\right|
$$

Para descobrirmos se $\hat{\rho}_{\lambda}\left(r_{j}, r_{j^{\prime}}\right)$ representa um estado puro ou não, devemos cacular $\operatorname{tr}\left(\hat{\rho}_{\lambda}\left(r_{j}, r_{j^{\prime}}\right)^{2}\right)$ :

$$
\begin{aligned}
\operatorname{tr}\left(\hat{\rho}_{\lambda}\left(r_{j}, r_{j^{\prime}}\right)^{2}\right) & =N^{4} \sum_{k, l, m} \sum_{k^{\prime}, l^{\prime}, m^{\prime}}\left(\phi_{k j} \phi_{m j^{\prime}} \phi_{l j} \phi_{m j^{\prime}}\right)\left(\phi_{k^{\prime} j} \phi_{m^{\prime} j^{\prime}} \phi_{l^{\prime} j} \phi_{m^{\prime} j^{\prime}}\right) \operatorname{tr}\left(\left|\omega_{k}, \lambda\right\rangle\left\langle\omega_{l}, \lambda \mid \omega_{k^{\prime}}, \lambda\right\rangle\left\langle\omega_{l^{\prime}}, \lambda\right|\right) \\
& =N^{4} \sum_{k, l, m} \sum_{k^{\prime}, l^{\prime}, m^{\prime}}\left(\phi_{k j} \phi_{m j^{\prime}} \phi_{l j} \phi_{m j^{\prime}}\right)\left(\phi_{k^{\prime} j} \phi_{m^{\prime} j^{\prime}} \phi_{l^{\prime} j} \phi_{m^{\prime} j^{\prime}}\right) \delta_{k l^{\prime}} \delta_{l k^{\prime}} \\
& =N^{4} \sum_{k, l, m, m^{\prime}} \phi_{k j} \phi_{m j^{\prime}} \phi_{l j} \phi_{m j^{\prime}} \phi_{l j} \phi_{m^{\prime} j^{\prime}} \phi_{k j} \phi_{m^{\prime} j^{\prime}} \\
\operatorname{tr}\left(\hat{\rho}_{\lambda}\left(r_{j}, r_{j^{\prime}}\right)^{2}\right) & =N^{4} \underbrace{\left[\sum_{k, m}\left(\phi_{k j} \phi_{m j^{\prime}}\right)^{2}\right]}_{N^{-2}} \underbrace{\left[\sum_{l, m^{\prime}}\left(\phi_{l j} \phi_{m^{\prime} j^{\prime}}\right)^{2}\right]}_{N^{-2}}=1 .
\end{aligned}
$$

Portanto, concluímos que $\operatorname{tr}\left(\hat{\rho}_{\lambda}\left(r_{j}, r_{j^{\prime}}\right)^{2}\right)=1$, o que nos diz que a matriz densidade reduzida representa um estado puro e, logo, $\left|r_{j}, \lambda ; r_{j^{\prime}}, \lambda^{\prime}\right\rangle$ não apresenta emaranhamento entre frequências.

\subsubsection{Estado de Dois Quasifótons na Presença de Campo Magnético Externo}

Quando tratamos do caso em que temos um campo magnético externo constante na direção de propagação dos fótons, os coeficientes das expressões em (4.1.1) passam a depender da polarização dos fótons, i.e.:

$$
c_{j, \lambda}=\sum_{i, \sigma}\left(a_{i, \sigma} \phi_{i \sigma, j \lambda}-a_{i, \sigma}^{\dagger} \psi_{i \sigma, j \lambda}\right) \quad \text { e } \quad c_{j, \lambda}^{\dagger}=\sum_{i, \sigma}\left(a_{i, \sigma}^{\dagger} \phi_{i \sigma, j \lambda}-a_{i, \sigma} \psi_{i \sigma, j \lambda}\right), \quad i=0,1,2
$$


onde os coeficientes $\phi_{i \sigma, j \lambda}$ e $\psi_{i \sigma, j \lambda}{ }^{*}$ dependem do momento $p$ dos elétrons, da densidade $\rho$ de elétrons, das frequências $\omega_{1}$ e $\omega_{2}$ dos fótons, da constante de estrutura fina $\alpha$, do campo magnético $B$ e das polarizações $\sigma$ e $\lambda$. Explicitamente, temos:

$$
\begin{aligned}
\phi_{i \sigma, j \lambda}= & \left\{\left[\left(\frac{\omega_{i}}{r_{j \lambda}}\right)^{1 / 2}+\left(\frac{r_{j \lambda}}{\omega_{i}}\right)^{1 / 2}\right] \frac{(3-2 \lambda)(2-\sigma)-i(\lambda-1)}{2\left(r_{j \lambda}^{2}-\omega_{i}^{2}\right)}\left(1-\delta_{0 i}\right)+\right. \\
& \left.+(\sigma-1)(\lambda-1)\left[\frac{(n p) E_{0}}{\alpha \rho} \frac{1}{r_{j \lambda}^{3}}\right]^{1 / 2} \delta_{0 i}\right\}\left[1+(\lambda-2) \delta_{0 j}\right] S_{j \lambda} \\
\psi_{i \sigma, j \lambda}= & \left\{\left[\left(\frac{\omega_{i}}{r_{j \lambda}}\right)^{1 / 2}-\left(\frac{r_{j \lambda}}{\omega_{i}}\right)^{1 / 2}\right] \frac{(3-2 \lambda)(2-\sigma)-i(\lambda-1)}{2\left(r_{j \lambda}^{2}-\omega_{i}^{2}\right)}\left(1-\delta_{0 i}\right)+\right. \\
& \left.+(\sigma-1)(2-\lambda)\left[\frac{(n p) E_{0}}{\alpha \rho} \frac{1}{r_{j \lambda}^{3}}\right]^{1 / 2} \delta_{0 i}\right\}\left[1+(\lambda-2) \delta_{0 j}\right] S_{j \lambda} \\
S_{j \lambda}= & {\left[\frac{(2 \lambda-3)(n p) E_{0}}{\alpha \rho} \frac{1}{r_{j \lambda}^{3}}+2 \sum_{j}\left(r_{j \lambda}^{2}-\omega_{j}^{2}\right)^{-2}\right]^{1 / 2} } \\
= & {\left[(2 \lambda-3) \frac{\alpha \rho}{(n p)} r_{j \lambda}^{3} \frac{\partial r_{j \lambda}}{\partial E_{0}}\right]^{1 / 2}, \quad E_{0}=\frac{|e| B}{(n p)} . }
\end{aligned}
$$

Considerando o fato de que o vácuo de quasifótons $|\Omega\rangle$ é aproximadamente igual ao vácuo de fótons $|0\rangle$, escrevemos o estado de dois quasifótons:

$$
\begin{aligned}
\left|r_{j \lambda}, r_{j^{\prime} \lambda^{\prime}}\right\rangle & =N c_{j \lambda}^{\dagger} c_{j^{\prime} \lambda^{\prime}}^{\dagger}|0\rangle \\
& =N \sum_{i, \sigma}\left(a_{i, \sigma}^{\dagger} \phi_{i \sigma, j \lambda}-a_{i, \sigma} \psi_{i \sigma, j \lambda}\right) \sum_{i^{\prime}, \sigma^{\prime}}\left(a_{i^{\prime}, \sigma^{\prime}}^{\dagger} \phi_{i^{\prime} \sigma^{\prime}, j^{\prime} \lambda^{\prime}}-a_{i^{\prime}, \sigma^{\prime}} \psi_{i^{\prime} \sigma^{\prime}, j^{\prime} \lambda^{\prime}}\right)|0\rangle \\
& =N \sum_{i, i^{\prime}} \sum_{\sigma, \sigma^{\prime}}\left(\phi_{i \sigma, j \lambda} \phi_{i^{\prime} \sigma^{\prime}, j^{\prime} \lambda^{\prime}} a_{i, \sigma}^{\dagger} a_{i^{\prime}, \sigma^{\prime}}^{\dagger}-\psi_{i \sigma, j \lambda} \phi_{i^{\prime} \sigma^{\prime}, j^{\prime} \lambda^{\prime}} a_{i, \sigma} a_{i^{\prime}, \sigma^{\prime}}^{\dagger}\right)|0\rangle \\
& =N \sum_{i, i^{\prime}} \sum_{\sigma, \sigma^{\prime}}\left(\phi_{i \sigma, j \lambda} \phi_{i^{\prime} \sigma^{\prime}, j^{\prime} \lambda^{\prime}} a_{i, \sigma}^{\dagger} a_{i^{\prime}, \sigma^{\prime}}^{\dagger}-\psi_{i \sigma, j \lambda} \phi_{i^{\prime} \sigma^{\prime}, j^{\prime} \lambda^{\prime}} \delta_{i i^{\prime}} \delta_{\sigma \sigma^{\prime}}\right)|0\rangle \\
\left|r_{j \lambda}, r_{j^{\prime} \lambda^{\prime}}\right\rangle & =N\left(\sum_{i, i^{\prime}} \sum_{\sigma, \sigma^{\prime}} \phi_{i \sigma, j \lambda} \phi_{i^{\prime} \sigma^{\prime}, j^{\prime} \lambda^{\prime}} a_{i, \sigma}^{\dagger} a_{i^{\prime}, \sigma^{\prime}}^{\dagger}|0\rangle-\sum_{i, \sigma} \psi_{i \sigma, j \lambda} \phi_{i \sigma, j^{\prime} \lambda^{\prime}}|0\rangle\right)
\end{aligned}
$$

Observe que o estado $\left|r_{j \lambda}, r_{j^{\prime} \lambda^{\prime}}\right\rangle$ apresenta parcelas que não correspondem a estados de dois fótons. De fato, constatamos a presença de uma amplitude associada ao vácuo, e também de amplitudes associadas a excitações no movimento do elétron gerado pela presença de campo

*Diferentemente do caso sem campo magnético, agora os índices $i$ e $j$ relativos às frequências dos fótons variam de 0 a 2 , porém $\omega_{0}=0$. O valor adicional 0 aparece porque o movimento ciclotrônico do elétron na presença do campo magnético constante é tratado como um quasifóton. 
magnético, o que conhecemos no caso não relativístico como níveis de Landau. Acreditamos que nosso modelo tenha inconsistências e seja válido apenas na região de parâmetros em que as amplitudes que não sejam de dois fótons de frequências distintas sejam desprezíveis. Dessa forma, esperamos que as amplitudes relevantes do estado $\left|r_{j \lambda}, r_{j^{\prime} \lambda^{\prime}}\right\rangle$ sejam apenas aquelas associadas com dois fótons de frequências distintas, o que pode ser escrito da forma:

$$
\left|r_{j \lambda}, r_{j^{\prime} \lambda^{\prime}}\right\rangle=N \sum_{i, i^{\prime}=1}^{2} \sum_{\sigma, \sigma^{\prime}=1}^{2}\left(1-\delta_{i i^{\prime}}\right) \phi_{i \sigma, j \lambda} \phi_{i^{\prime} \sigma^{\prime}, j^{\prime} \lambda^{\prime}} a_{i, \sigma}^{\dagger} a_{i^{\prime}, \sigma^{\prime}}^{\dagger}|0\rangle,
$$

onde $N$ é uma constante de normalização. Gostaríamos de saber quando $\left|r_{j \lambda}, r_{j^{\prime} \lambda^{\prime}}\right\rangle$ representa um estado emaranhado. Primeiramente, gostaríamos de usar uma notação que seja capaz de explicitar imediatamente que temos dois subsistemas, um associado à frequência $\omega_{1}$ e outro associado à frequência $\omega_{2}$. Para tanto, reescrevemos $\left|r_{j \lambda}, r_{j^{\prime} \lambda^{\prime}}\right\rangle$ explicitando as somas em $i$ e $i^{\prime}:$

$$
\begin{aligned}
\left|r_{j \lambda}, r_{j^{\prime} \lambda^{\prime}}\right\rangle & =N \sum_{\sigma, \sigma^{\prime}}\left(\phi_{1 \sigma, j \lambda} \phi_{2 \sigma^{\prime}, j^{\prime} \lambda^{\prime}}\left|\sigma, \sigma^{\prime}\right\rangle+\phi_{2 \sigma, j \lambda} \phi_{1 \sigma^{\prime}, j^{\prime} \lambda^{\prime}}\left|\sigma^{\prime}, \sigma\right\rangle\right) \\
& =N \sum_{\sigma, \sigma^{\prime}}\left(\phi_{1 \sigma, j \lambda} \phi_{2 \sigma^{\prime}, j^{\prime} \lambda^{\prime}}+\phi_{2 \sigma^{\prime}, j \lambda} \phi_{1 \sigma, j^{\prime} \lambda^{\prime}}\right)\left|\sigma, \sigma^{\prime}\right\rangle .
\end{aligned}
$$

No ket $\left|\sigma, \sigma^{\prime}\right\rangle$, a primeira posição está associada à frequência $\omega_{1}$ e a segunda à frequência $\omega_{2}$, de forma que:

$$
\left|\sigma, \sigma^{\prime}\right\rangle \equiv a_{1, \sigma}^{\dagger} a_{2, \sigma^{\prime}}^{\dagger}|0\rangle
$$

Por simplicidade, definimos:

$$
\alpha_{a b}\left(j, \lambda ; j^{\prime}, \lambda^{\prime}\right):=\phi_{1 a, j \lambda} \phi_{2 b, j^{\prime} \lambda^{\prime}}+\phi_{2 b, j \lambda} \phi_{1 a, j^{\prime} \lambda^{\prime}}, \quad a, b=1,2 .
$$

Usando essa notação, o estado $\left|r_{j \lambda}, r_{j^{\prime} \lambda^{\prime}}\right\rangle$ se escreve:

$$
\left|r_{j \lambda}, r_{j^{\prime} \lambda^{\prime}}\right\rangle=N \sum_{a, b} \alpha_{a b}|a, b\rangle, \quad N=\left(\sum_{a, b} \alpha_{a b}^{2}\right)^{-1 / 2}
$$




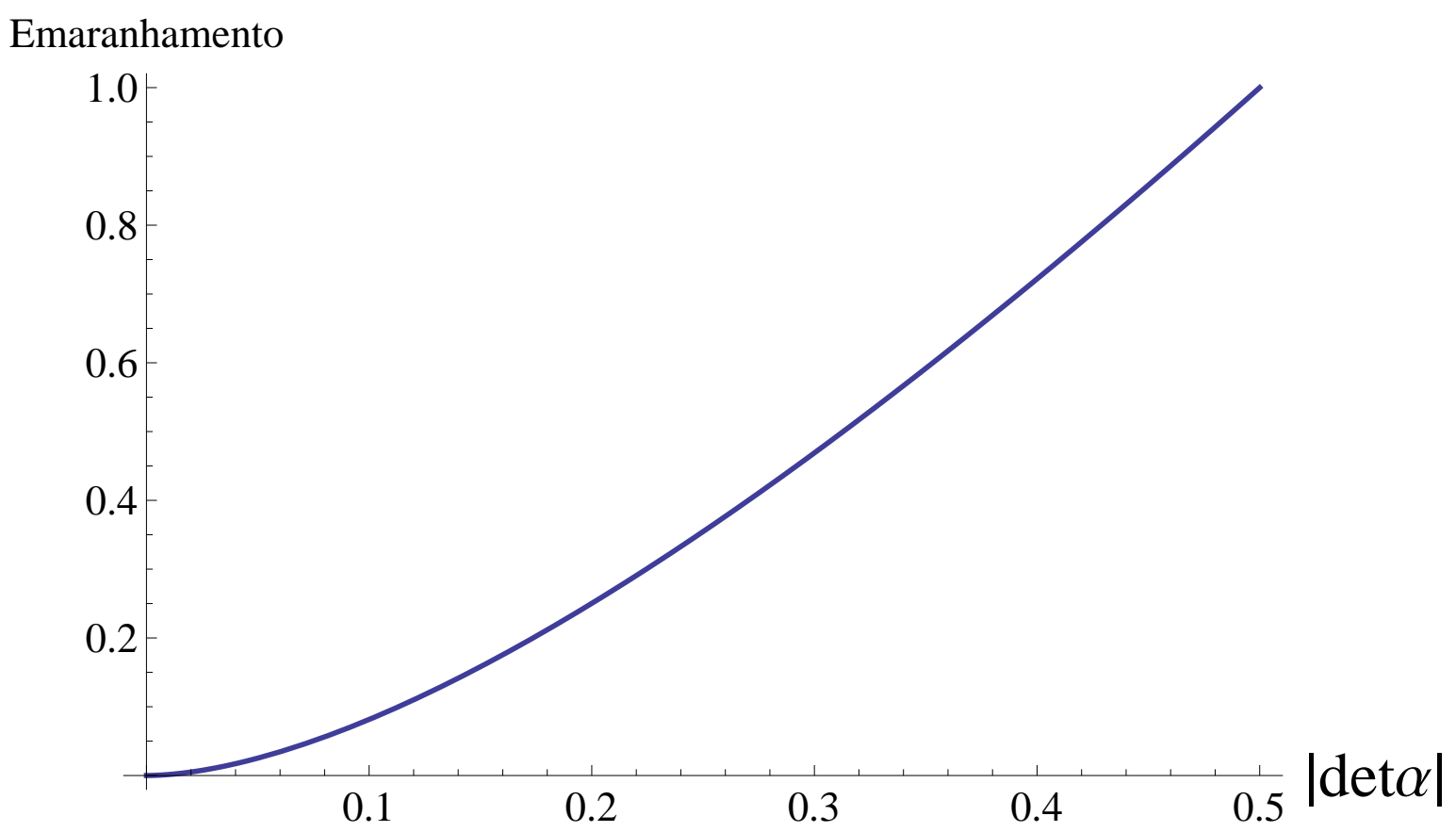

Figura 4.1.1: Gráfico da quantidade de emaranhamento do estado $\left|r_{j \lambda}, r_{j^{\prime} \lambda^{\prime}}\right\rangle$ em termos do determinante da matriz $\boldsymbol{\alpha}$.

Usando o resultado obtido na seção (3.4) temos prontamente que a quantidade de emaranhamento contida nesse estado é dada por

$$
\begin{gathered}
E\left(\left|r_{j \lambda}, r_{j^{\prime} \lambda^{\prime}}\right\rangle\right)=-\left[\log _{2}|\operatorname{det} \boldsymbol{\alpha}|+\sqrt{1-4|\operatorname{det} \boldsymbol{\alpha}|^{2}} \log _{2}\left(\frac{1+\sqrt{1-4|\operatorname{det} \boldsymbol{\alpha}|^{2}}}{2|\operatorname{det} \boldsymbol{\alpha}|}\right)\right] \\
0 \leq|\operatorname{det} \boldsymbol{\alpha}| \leq \frac{1}{2}
\end{gathered}
$$

\subsection{Emaranhamento com Vácuo de Quasifótons Exato}

Nessa seção vamos considerar o caso monocromático, i.e. só trataremos de fótons e quasifótons com uma frequência $\omega$ específica, na ausência de campo externo. Nesse caso é fácil tratar exatamente o vácuo de quasifótons, o que é algo interessante pois nos permite estudar situações com energias arbitrariamente elevadas.

Seja $c_{i}$ o operador de aniquilação de quasifótons. Escrevendo $c_{i}$ em termos dos operadores 
$b_{i}^{\dagger}$ e $b_{i}$ de criação e aniquilação de fótons em polarização linear, temos:

$$
\begin{aligned}
& c_{1}=\phi b_{1}-\psi b_{1}^{\dagger}, \\
& c_{2}=\phi b_{2}-\psi b_{2}^{\dagger},
\end{aligned}
$$

onde $\phi$ e $\psi$, segundo as equações (2.2.24)-(2.2.26), são dados por:

$$
\begin{aligned}
\phi & =\frac{1}{2}\left[\sqrt{\frac{\omega}{r}}+\sqrt{\frac{r}{\omega}}\right], \\
\psi & =\frac{1}{2}\left[\sqrt{\frac{\omega}{r}}-\sqrt{\frac{r}{\omega}}\right] .
\end{aligned}
$$

Da equação (2.2.28) obtemos uma expressão para $r$ :

$$
r=\sqrt{\omega^{2}+\alpha \frac{\rho}{(n p)}} .
$$

Como anteriormente, o termo linear pode ser colocado à zero através de uma escolha conveniente de referencial. Podemos igualmente escrever $c_{i}$ em termos dos operadores $a_{i}^{\dagger}$ e $a_{i}$ de criação e aniquilação de fótons em polarização circular, lembrando que:

$$
\begin{aligned}
& \left(\begin{array}{l}
a_{1}^{\dagger} \\
a_{2}^{\dagger}
\end{array}\right)=\frac{1}{\sqrt{2}}\left(\begin{array}{cc}
1 & i \\
1 & -i
\end{array}\right)\left(\begin{array}{l}
b_{1}^{\dagger} \\
b_{2}^{\dagger}
\end{array}\right) \Leftrightarrow\left(\begin{array}{l}
b_{1}^{\dagger} \\
b_{2}^{\dagger}
\end{array}\right)=\frac{1}{\sqrt{2}}\left(\begin{array}{cc}
1 & 1 \\
-i & i
\end{array}\right)\left(\begin{array}{l}
a_{1}^{\dagger} \\
a_{2}^{\dagger}
\end{array}\right), \\
& \left(\begin{array}{l}
a_{1} \\
a_{2}
\end{array}\right)=\frac{1}{\sqrt{2}}\left(\begin{array}{cc}
1 & -i \\
1 & i
\end{array}\right)\left(\begin{array}{l}
b_{1} \\
b_{2}
\end{array}\right) \Leftrightarrow\left(\begin{array}{l}
b_{1} \\
b_{2}
\end{array}\right)=\frac{1}{\sqrt{2}}\left(\begin{array}{cc}
1 & 1 \\
i & -i
\end{array}\right)\left(\begin{array}{l}
a_{1} \\
a_{2}
\end{array}\right),
\end{aligned}
$$

onde implicitamente reside a hipótese de que o vácuo de fótons de polarização linear e circular é o mesmo, i.e.:

$$
a_{i}|0\rangle=b_{j}|0\rangle=0, \quad \forall i, j
$$

Escrevendo $c_{i}$ em termos de fótons de polarização circular, temos:

$$
\begin{aligned}
c_{1} & =\frac{\phi}{\sqrt{2}}\left(a_{1}+a_{2}\right)-\frac{\psi}{\sqrt{2}}\left(a_{1}^{\dagger}+a_{2}^{\dagger}\right), \\
c_{2} & =\frac{i \phi}{\sqrt{2}}\left(a_{1}-a_{2}\right)-\frac{i \psi}{\sqrt{2}}\left(a_{2}^{\dagger}-a_{1}^{\dagger}\right) .
\end{aligned}
$$

Estamos interessados em descobrir uma expressão explícita para o estado $|\Omega\rangle$ que satisfaz:

$$
c_{i}|\Omega\rangle=0, \quad \forall i
$$


Gostaríamos de enfatizar o fato de que o estado $|\Omega\rangle$ depende crucialmente da escolha de polarização dos fótons, i.e. linear ou circular. Para encontrar a solução de (4.2.3) vamos utilizar a representação de Fock-Bargmann (vide apêndice D).

\section{Fótons de Polarização Linear}

$$
\left\{\begin{array}{l}
\left(\phi \frac{\partial}{\partial z_{1}}-\psi z_{1}\right) \Omega\left(z_{1}, z_{2}\right)=0 \\
\left(\phi \frac{\partial}{\partial z_{2}}-\psi z_{2}\right) \Omega\left(z_{1}, z_{2}\right)=0
\end{array} \quad \Rightarrow \Psi\left(z_{1}, z_{2}\right)=k e^{\frac{1}{2} \frac{\psi}{\phi} z_{1}^{2}} e^{\frac{1}{2} \frac{\psi}{\phi} z_{2}^{2}}\right.
$$

Voltando para a representação abstrata:

$$
\left|\Omega_{00}^{l}\right\rangle=k e^{\frac{1}{2} \frac{\psi}{\phi}\left(b_{1}^{\dagger}\right)^{2}} e^{\frac{1}{2} \frac{\psi}{\phi}\left(b_{2}^{\dagger}\right)^{2}}|0,0\rangle .
$$

Fótons de Polarização Circular

$$
\left\{\begin{array} { l } 
{ \frac { 1 } { \sqrt { 2 } } [ \phi ( \frac { \partial } { \partial z _ { 1 } } + \frac { \partial } { \partial z _ { 2 } } ) - \psi ( z _ { 1 } + z _ { 2 } ) ] \Omega ( z _ { 1 } , z _ { 2 } ) = 0 } \\
{ \frac { i } { \sqrt { 2 } } [ \phi ( \frac { \partial } { \partial z _ { 1 } } - \frac { \partial } { \partial z _ { 2 } } ) - \psi ( z _ { 2 } - z _ { 1 } ) ] \Omega ( z _ { 1 } , z _ { 2 } ) = 0 }
\end{array} \Rightarrow \left\{\begin{array}{l}
\left(\phi \frac{\partial}{\partial z_{1}}-\psi z_{2}\right) \Omega\left(z_{1}, z_{2}\right)=0 \\
\left(\phi \frac{\partial}{\partial z_{2}}-\psi z_{1}\right) \Omega\left(z_{1}, z_{2}\right)=0
\end{array}\right.\right.
$$

Essas equações nos levam a concluir que

$$
\Omega\left(z_{1}, z_{2}\right)=k e^{\frac{\psi}{\phi} z_{1} z_{2}}, \quad k \in \mathbb{C},
$$

que, na representação abstrata, se escreve como

$$
\left|\Omega_{00}^{c}\right\rangle=k e^{\frac{\psi}{\phi} a_{1}^{\dagger} a_{2}^{\dagger}}|0,0\rangle .
$$

Note que podemos transformar $\left|\Omega_{00}^{c}\right\rangle$ em $\left|\Omega_{00}^{l}\right\rangle$, e vice-versa, utilizando as transformações (4.2.1) diretamente em (4.2.4) ou (4.2.5), i.e.:

$$
e^{\frac{\psi}{\phi} a_{1}^{\dagger} a_{2}^{\dagger}}=\exp \left[\frac{\psi}{\phi}\left(\frac{b_{1}^{\dagger}+i b_{2}^{\dagger}}{\sqrt{2}}\right)\left(\frac{b_{1}^{\dagger}-i b_{2}^{\dagger}}{\sqrt{2}}\right)\right]=e^{\frac{1}{2} \frac{\psi}{\phi}\left(b_{1}^{\dagger}\right)^{2}} e^{\frac{1}{2} \frac{\psi}{\phi}\left(b_{2}^{\dagger}\right)^{2}}
$$

Note, porém, que os estados $\left|\Omega_{00}^{l}\right\rangle$ e $\left|\Omega_{00}^{c}\right\rangle$ apresentam características distintas, em particular, $\left|\Omega_{00}^{l}\right\rangle$ é um estado separado, pois é simplesmente o produto tensorial de dois estados, i.e. $\left|\Omega_{00}^{l}\right\rangle \propto\left(e^{\frac{1}{2} \frac{\psi}{\phi}\left(b_{1}^{\dagger}\right)^{2}}|0\rangle\right) \otimes\left(e^{\frac{1}{2} \frac{\psi}{\phi}\left(b_{2}^{\dagger}\right)^{2}}|0\rangle\right)$, enquanto que o estado $\left|\Omega_{00}^{c}\right\rangle$ é um estado 
emaranhado cuja quantidade de emaranhamento é

$$
\begin{gathered}
E\left(\Omega_{00}^{c}\right)=\cosh ^{2} r \log _{2}\left(\cosh ^{2} r\right)-\sinh ^{2} r \log _{2}\left(\sinh ^{2} r\right), \\
r=\frac{1}{2} \ln \left(\frac{\phi+\psi}{\phi-\psi}\right) .
\end{gathered}
$$

O leitor pode considerar curioso que o vácuo de quasifótons estar emaranhado dependa da escolha de polarização que façamos, porém esse resultado é apenas mais um exemplo de que o emaranhamento de um sistema físico depende do conjunto de observáveis considerado, como é defendido em [27]. A ideia é que o conceito de emaranhamento depende de podermos distinguir ao menos dois subsistemas num sistema quântico, o que é feito através de um certo conjunto de observáveis. Dessa forma, dado um conjunto de observáveis, temos uma forma de fatorar o espaço de Hilbert no qual o sistema quântico é descrito. No entanto, caso tivéssemos escolhido um outro conjunto de observáveis, teríamos uma nova forma de fatorar o espaço de Hilbert e não necessariamente estados que eram emaranhados antes continuarão emaranhados. Para ilustrar o que foi dito com mais um caso concreto, lembre-se do átomo de hidrogênio. O modelo mais simples para o átomo de hidrogênio consiste em duas partículas puntiformes, carregadas e ligadas pela interação coulombiana. O estado desse sistema escrito em termos das posições dessas partículas é um estado emaranhado, porém, se descrevermos esse mesmo sistema em termos de posição do centro de massa e posição relativa, o mesmo estado passa a ser separável, visto que em relação a esse novo conjunto de observáveis o sistema é não interagente.

Gostaríamos de introduzir o conceito da polarização dos quasifótons. Definiremos que quasifótons de polarização linear estão associados aos operadores $c_{i}$ e $c_{i}^{\dagger}$. Usando as transformações (4.2.1) definimos os operadores $d_{i}$ e $d_{i}^{\dagger}$ associados a quasifótons de polarização circular:

$$
\begin{aligned}
\left(\begin{array}{l}
d_{1}^{\dagger} \\
d_{2}^{\dagger}
\end{array}\right) & =\frac{1}{\sqrt{2}}\left(\begin{array}{cc}
1 & i \\
1 & -i
\end{array}\right)\left(\begin{array}{c}
c_{1}^{\dagger} \\
c_{2}^{\dagger}
\end{array}\right) \Leftrightarrow\left(\begin{array}{c}
c_{1}^{\dagger} \\
c_{2}^{\dagger}
\end{array}\right)=\frac{1}{\sqrt{2}}\left(\begin{array}{cc}
1 & 1 \\
-i & i
\end{array}\right)\left(\begin{array}{c}
d_{1}^{\dagger} \\
d_{2}^{\dagger}
\end{array}\right), \\
\left(\begin{array}{l}
d_{1} \\
d_{2}
\end{array}\right) & =\frac{1}{\sqrt{2}}\left(\begin{array}{cc}
1 & -i \\
1 & i
\end{array}\right)\left(\begin{array}{l}
c_{1} \\
c_{2}
\end{array}\right) \Leftrightarrow\left(\begin{array}{c}
c_{1} \\
c_{2}
\end{array}\right)=\frac{1}{\sqrt{2}}\left(\begin{array}{cc}
1 & 1 \\
i & -i
\end{array}\right)\left(\begin{array}{c}
d_{1} \\
d_{2}
\end{array}\right),
\end{aligned}
$$

onde, mais uma vez ressaltamos, reside implicitamente a hipótese de que o vácuo de quasifótons de polarização linear e circular é o mesmo, i.e.:

$$
c_{i}|\Omega\rangle=d_{j}|\Omega\rangle=0, \quad \forall i, j
$$


Observe que se escrevermos os operadores de aniquilação de quasifótons de polarização circular em termos de operadores de criação e aniquilação de fótons de polarização linear, temos:

$$
\begin{aligned}
& d_{1}=\frac{1}{\sqrt{2}}\left(c_{1}-i c_{2}\right)=\frac{1}{\sqrt{2}}\left[\phi b_{1}-\psi b_{1}^{\dagger}-i\left(\phi b_{2}-\psi b_{2}^{\dagger}\right)\right]=\phi\left(\frac{b_{1}-i b_{2}}{\sqrt{2}}\right)-\psi\left(\frac{b_{1}^{\dagger}-i b_{2}^{\dagger}}{\sqrt{2}}\right) \\
& d_{2}=\frac{1}{\sqrt{2}}\left(c_{1}+i c_{2}\right)=\frac{1}{\sqrt{2}}\left[\phi b_{1}-\psi b_{1}^{\dagger}+i\left(\phi b_{2}-\psi b_{2}^{\dagger}\right)\right]=\phi\left(\frac{b_{1}+i b_{2}}{\sqrt{2}}\right)-\psi\left(\frac{b_{1}^{\dagger}+i b_{2}^{\dagger}}{\sqrt{2}}\right)
\end{aligned}
$$

Enquanto que em termos de operadores de criação e aniquilação de fótons de polarização circular, temos:

$$
\begin{aligned}
d_{1}= & \frac{1}{\sqrt{2}}\left(c_{1}-i c_{2}\right)=\frac{1}{\sqrt{2}}\left[\frac{\phi}{\sqrt{2}}\left(a_{1}+a_{2}\right)-\frac{\psi}{\sqrt{2}}\left(a_{1}^{\dagger}+a_{2}^{\dagger}\right)-\right. \\
& \left.-i\left(\frac{i \phi}{\sqrt{2}}\left(a_{1}-a_{2}\right)-\frac{i \psi}{\sqrt{2}}\left(a_{2}^{\dagger}-a_{1}^{\dagger}\right)\right)\right] \\
d_{1}= & \phi a_{1}-\psi a_{2}^{\dagger}, \\
d_{2}= & \frac{1}{\sqrt{2}}\left(c_{1}+i c_{2}\right)=\frac{1}{\sqrt{2}}\left[\frac{\phi}{\sqrt{2}}\left(a_{1}+a_{2}\right)-\frac{\psi}{\sqrt{2}}\left(a_{1}^{\dagger}+a_{2}^{\dagger}\right)+\right. \\
& \left.-i\left(\frac{i \phi}{\sqrt{2}}\left(a_{1}-a_{2}\right)-\frac{i \psi}{\sqrt{2}}\left(a_{2}^{\dagger}-a_{1}^{\dagger}\right)\right)\right] \\
d_{2}= & \phi a_{2}-\psi a_{1}^{\dagger} .
\end{aligned}
$$

Ou seja, a relação entre quasifótons de polarização circular e fótons de polarização circular é

$$
\left\{\begin{array}{l}
d_{1}=\phi a_{1}-\psi a_{2}^{\dagger} \\
d_{2}=\phi a_{2}-\psi a_{1}^{\dagger}
\end{array} .\right.
$$

Escrevendo a equação

$$
d_{i}|\Omega\rangle=0
$$

em representação de Fock-Bargmann nós temos:

\section{Fótons de Polarização Linear}

$$
\left\{\begin{array} { l } 
{ [ \phi ( \frac { \partial } { \partial z _ { 1 } } - i \frac { \partial } { \partial z _ { 2 } } ) - \psi ( z _ { 1 } - i z _ { 2 } ) ] \Omega ( z _ { 1 } , z _ { 2 } ) = 0 } \\
{ [ \phi ( \frac { \partial } { \partial z _ { 1 } } + i \frac { \partial } { \partial z _ { 2 } } ) - \psi ( z _ { 2 } + i z _ { 1 } ) ] \Omega ( z _ { 1 } , z _ { 2 } ) = 0 }
\end{array} \Rightarrow \left\{\begin{array}{l}
\left(\phi \frac{\partial}{\partial z_{1}}-\psi z_{1}\right) \Omega\left(z_{1}, z_{2}\right)=0 \\
\left(\phi \frac{\partial}{\partial z_{2}}-\psi z_{2}\right) \Omega\left(z_{1}, z_{2}\right)=0
\end{array}\right.\right.
$$




$$
\Omega\left(z_{1}, z_{2}\right)=k e^{\frac{1}{2} \frac{\psi}{\phi} z_{1}^{2}} e^{\frac{1}{2} \frac{\psi}{\phi} z_{2}^{2}}
$$

o que na representação abstrata adquire a forma

$$
\left|\Omega_{00}^{l}\right\rangle=k e^{\frac{1}{2} \frac{\psi}{\phi}\left(b_{1}^{\dagger}\right)^{2}} e^{\frac{1}{2} \frac{\psi}{\phi}\left(b_{2}^{\dagger}\right)^{2}}|0,0\rangle .
$$

Fótons de Polarização Circular

$$
\left\{\begin{array}{l}
\left(\phi \frac{\partial}{\partial z_{1}}-\psi z_{2}\right) \Omega\left(z_{1}, z_{2}\right)=0 \\
\left(\phi \frac{\partial}{\partial z_{2}}-\psi z_{1}\right) \Omega\left(z_{1}, z_{2}\right)=0
\end{array} \quad \Rightarrow \Omega\left(z_{1}, z_{2}\right)=k e^{\frac{\psi}{\phi} z_{1} z_{2}}\right.
$$

que pode ser expresso como

$$
\left|\Omega_{00}^{c}\right\rangle=k e^{\frac{\psi}{\phi} a_{1}^{\dagger} a_{2}^{\dagger}}|0,0\rangle .
$$

Esses resultados estão de acordo com o esperado, i.e. ao fixarmos uma base de polarização para os fótons, fixamos as propriedades do vácuo de quasifótons, independentemente de usarmos quasifótons de polarização linear ou circular.

\subsubsection{Emaranhamento de Estados de Dois Quasifótons}

Agora estamos interessados em analisar o estado de dois quasifótons de polarizações distintas. Assumindo a base de polarização circular para os fótons, fixamos $\left|\Omega_{00}^{c}\right\rangle$ como o estado de vácuo de quasifótons. Os estados que nos interessam no momento são:

$$
\left|\Phi_{11}^{l i n}\right\rangle=c_{1}^{\dagger} c_{2}^{\dagger}\left|\Omega_{00}^{c}\right\rangle \quad \text { e } \quad\left|\Phi_{11}^{c i r c}\right\rangle=d_{1}^{\dagger} d_{2}^{\dagger}\left|\Omega_{00}^{c}\right\rangle
$$

Escrevendo os operadores $c_{i}^{\dagger}$ e $d_{i}^{\dagger}$ em termos de operadores de criação e aniquilação de fótons de polarização circular, temos:

$$
\begin{aligned}
\left|\Phi_{11}^{l i n}\right\rangle & =\left(\frac{\phi}{\sqrt{2}}\left(a_{1}+a_{2}\right)-\frac{\psi}{\sqrt{2}}\left(a_{1}^{\dagger}+a_{2}^{\dagger}\right)\right)\left(\frac{i \phi}{\sqrt{2}}\left(a_{1}-a_{2}\right)-\frac{i \psi}{\sqrt{2}}\left(a_{2}^{\dagger}-a_{1}^{\dagger}\right)\right)\left|\Omega_{00}^{c}\right\rangle \\
& =\frac{i}{2}\left[\phi^{2}\left(a_{1}^{2}-a_{2}^{2}\right)+\psi^{2}\left(\left(a_{1}^{\dagger}\right)^{2}-\left(a_{2}^{\dagger}\right)^{2}\right)-2 \phi \psi\left(a_{1} a_{2}^{\dagger}-a_{2} a_{1}^{\dagger}\right)\right]\left|\Omega_{00}^{c}\right\rangle
\end{aligned}
$$

$\mathrm{e}$

$$
\begin{aligned}
\left|\Phi_{11}^{c i r c}\right\rangle & =\left(\phi a_{1}^{\dagger}-\psi a_{2}\right)\left(\phi a_{2}^{\dagger}-\psi a_{1}\right)\left|\Omega_{00}^{c}\right\rangle \\
& =\left[\phi^{2} a_{1}^{\dagger} a_{2}^{\dagger}+\psi^{2} a_{1} a_{2}-\phi \psi\left(a_{1}^{\dagger} a_{1}+a_{2}^{\dagger} a_{2}+1\right)\right]\left|\Omega_{00}^{c}\right\rangle .
\end{aligned}
$$


Os estados $\left|\Phi_{11}^{\text {lin }}\right\rangle$ e $\left|\Phi_{11}^{\text {circ }}\right\rangle$, podem, certamente, ser escritos em termos de estados de número definido de fótons de polarização circular, i.e.:

$$
\begin{aligned}
& \left|\Phi_{11}^{l i n}\right\rangle=\alpha_{11}|1,1\rangle+\alpha_{20}|2,0\rangle+\alpha_{02}|0,2\rangle+\cdots \\
& \left|\Phi_{11}^{\text {circ }}\right\rangle=\beta_{11}|1,1\rangle+\beta_{20}|2,0\rangle+\beta_{02}|0,2\rangle+\cdots .
\end{aligned}
$$

Vamos nos concentrar no cálculo das amplitudes de dois fótons apresentadas nas expressões (4.2.8) e (4.2.9). Primeiramente, lembramos que o estado

$$
\left|\Psi_{00}^{c}\right\rangle=k e^{\frac{\psi}{\phi} a_{1}^{\dagger} a_{2}^{\dagger}}|0,0\rangle
$$

pode ser escrito em termos do operador $S_{12}(r)$ de compressão de dois modos (vide apêndice F), i.e.:

$$
\left|\Omega_{00}^{c}\right\rangle=S_{12}(r)|0,0\rangle, \quad S_{12}(r)=\exp \left[r \hat{a}_{1}^{\dagger} \hat{a}_{2}^{\dagger}-r \hat{a}_{1} \hat{a}_{2}\right], r \in \mathbb{R},
$$

donde tiramos que

$$
\left|\Omega_{00}^{c}\right\rangle=\frac{1}{\cosh (r)} \sum_{n=0}^{\infty} \tanh ^{n}(r)|n, n\rangle .
$$

Cálculo das Amplitudes de Probabilidade no Caso de Quasifótons de Polarização Linear Para facilitar os cálculos, é conveniente calcular inicialmente a ação de $c_{1}^{\dagger} c_{2}^{\dagger} \mathrm{em}$ $|n, n\rangle$ :

$$
\begin{aligned}
c_{1}^{\dagger} c_{2}^{\dagger}|n, n\rangle= & \frac{i}{2}\left[\phi^{2}\left(a_{1}^{2}-a_{2}^{2}\right)+\psi^{2}\left(\left(a_{1}^{\dagger}\right)^{2}-\left(a_{2}^{\dagger}\right)^{2}\right)-2 \phi \psi\left(a_{1} a_{2}^{\dagger}-a_{2} a_{1}^{\dagger}\right)\right]|n, n\rangle \\
= & \frac{i}{2}\left[\phi^{2} \sqrt{n(n-1)}(|n-2, n\rangle-|n, n-2\rangle)+\right. \\
& +\psi^{2} \sqrt{(n+1)(n+2)}(|n+2, n\rangle-|n, n+2\rangle)- \\
& -2 \phi \psi \sqrt{n(n+1)}(|n-1, n+1\rangle-|n+1, n-1\rangle)] .
\end{aligned}
$$

Assim, temos que:

$$
\begin{aligned}
\left|\Phi_{11}^{l i n}\right\rangle= & \frac{i}{2 \cosh (r)}\left[\sum_{n=2}^{\infty} \phi^{2} \tanh ^{n}(r) \sqrt{n(n-1)}(|n-2, n\rangle-|n, n-2\rangle)+\right. \\
& +\sum_{n=0}^{\infty} \psi^{2} \tanh ^{n}(r) \sqrt{(n+1)(n+2)}(|n+2, n\rangle-|n, n+2\rangle)- \\
& \left.-\sum_{n=1}^{\infty} 2 \phi \psi \tanh ^{n}(r) \sqrt{n(n+1)}(|n-1, n+1\rangle-|n+1, n-1\rangle)\right]
\end{aligned}
$$


De forma que

$$
\begin{array}{r}
\left|\Phi_{11}^{l i n}\right\rangle=\frac{i}{2 \cosh (r)}\left[\sum_{n=0}^{\infty} \sqrt{(n+1)(n+2)}\left(\phi^{2} \tanh ^{n+2}(r)-\psi^{2} \tanh ^{n}(r)-2 \phi \psi \tanh ^{n+1}(r)\right) \times\right. \\
\times(|n, n+2\rangle-|n+2, n\rangle)]
\end{array}
$$

O cálculo das amplitudes $\alpha_{11}, \alpha_{20}$ e $\alpha_{02}$ segue facilmente de (4.2.11):

$$
\begin{gathered}
\alpha_{11}=\left\langle 1,1 \mid \Phi_{11}^{\text {lin }}\right\rangle=0, \\
\alpha_{20}=\left\langle 2,0 \mid \Phi_{11}^{l i n}\right\rangle=\frac{i}{\sqrt{2} \cosh (r)}\left[-\phi^{2} \tanh ^{2}(r)+\psi^{2}+2 \phi \psi \tanh (r)\right], \\
\alpha_{02}=\left\langle 0,2 \mid \Phi_{11}^{l i n}\right\rangle=-\frac{i}{\sqrt{2} \cosh (r)}\left[-\phi^{2} \tanh ^{2}(r)+\psi^{2}+2 \phi \psi \tanh (r)\right] .
\end{gathered}
$$

Essas expressões podem ser simplificadas se levarmos em conta o fato de que

$$
\frac{\psi}{\phi}=\tanh (r) \Rightarrow \cosh (r)=\frac{1}{\sqrt{1-\tanh ^{2}(r)}}=\frac{\phi}{\sqrt{\phi^{2}-\psi^{2}}} .
$$

Assim,

$$
\alpha_{20}=-\alpha_{02}=i \sqrt{2} \frac{\psi^{2}}{\phi} \sqrt{\phi^{2}-\psi^{2}} .
$$

Esse resultado nos diz que a probabilidade de $\left|\Phi_{11}^{\text {lin }}\right\rangle$ ser observado no estado $|1,1\rangle$ é nula, porém a probabilidade de ser observado em $|2,0\rangle$ é a mesma que a de ser observado em $|0,2\rangle$, que, em geral, é não nula.

\section{Cálculo das Amplitudes de Probabilidade no Caso de Quasifótons de Polarização}

Circular Procedendo da mesma forma que anteriormente,

$$
\begin{aligned}
d_{1}^{\dagger} d_{2}^{\dagger}|n, n\rangle & =\left[\phi^{2} a_{1}^{\dagger} a_{2}^{\dagger}+\psi^{2} a_{1} a_{2}-\phi \psi\left(a_{1}^{\dagger} a_{1}+a_{2}^{\dagger} a_{2}+1\right)\right]|n, n\rangle \\
& =\left[\phi^{2}(n+1)|n+1, n+1\rangle+\psi^{2} n|n-1, n-1\rangle-\phi \psi(2 n+1)|n, n\rangle\right] .
\end{aligned}
$$


Dessa forma,

$$
\begin{aligned}
\left|\Phi_{11}^{c i r c}\right\rangle= & \frac{1}{\cosh (r)}\left[\sum_{n=0}^{\infty} \tanh ^{n}(r) \phi^{2}(n+1)|n+1, n+1\rangle+\right. \\
& +\sum_{n=1}^{\infty} \tanh ^{n}(r) \psi^{2} n|n-1, n-1\rangle- \\
& \left.-\sum_{n=0}^{\infty} \tanh ^{n}(r) \phi \psi(2 n+1)|n, n\rangle\right] .
\end{aligned}
$$

Portanto, obtemos

$$
\begin{aligned}
\left|\Phi_{11}^{\text {circ }}\right\rangle= & \frac{1}{\cosh (r)}\left\{\sum_{n=0}^{\infty}\left[(n+1) \phi^{2} \tanh ^{n}(r)|n+1, n+1\rangle\right]\right. \\
& \left.+\sum_{n=0}^{\infty}\left[\left((n+1) \psi^{2} \tanh ^{n+1}(r)-(2 n+1) \phi \psi \tanh ^{n}(r)\right)|n, n\rangle\right]\right\} .
\end{aligned}
$$

O cálculo das amplitudes $\beta_{11}, \beta_{20}$ e $\beta_{02}$ segue facilmente de (4.2.13):

$$
\begin{gathered}
\beta_{11}=\left\langle 1,1 \mid \Phi_{11}^{\text {circ }}\right\rangle=\frac{1}{\cosh (r)}\left[\phi^{2}+2 \psi^{2} \tanh ^{2}(r)-3 \phi \psi \tanh (r)\right] \\
\beta_{20}=\left\langle 2,0 \mid \Phi_{11}^{\text {circ }}\right\rangle=0 \\
\beta_{02}=\left\langle 0,2 \mid \Phi_{11}^{\text {circ }}\right\rangle=0 .
\end{gathered}
$$

Usando as relações (4.2.12), obtemos:

$$
\beta_{11}=\frac{\phi}{\sqrt{\phi^{2}-\psi^{2}}}\left[\phi^{2}-3 \psi^{2}+2 \frac{\psi^{4}}{\phi^{2}}\right]
$$

Observamos na expressão (4.2.13) que a amplitude $\beta_{00}=\left\langle 0,0 \mid \Phi_{00}^{\text {circ }}\right\rangle$ é relevante. Calculandoa, obtemos:

$$
\beta_{00}=\frac{1}{\cosh (r)}\left[\psi^{2} \tanh (r)-\phi \psi\right] \Rightarrow \beta_{00}=\frac{\phi}{\sqrt{\phi^{2}-\psi^{2}}}\left[\frac{\psi^{3}}{\phi}-\phi \psi\right]
$$

Se fizermos um truncamento considerando apenas os termos mais significativos de (4.2.11) e (4.2.13), teremos:

$$
\left|\Phi_{11}^{\text {lin }}\right\rangle=\alpha_{20}|2,0\rangle+\alpha_{02}|0,2\rangle \quad \text { e }\left|\Phi_{11}^{\text {circ }}\right\rangle=\beta_{00}|0,0\rangle+\beta_{11}|1,1\rangle
$$




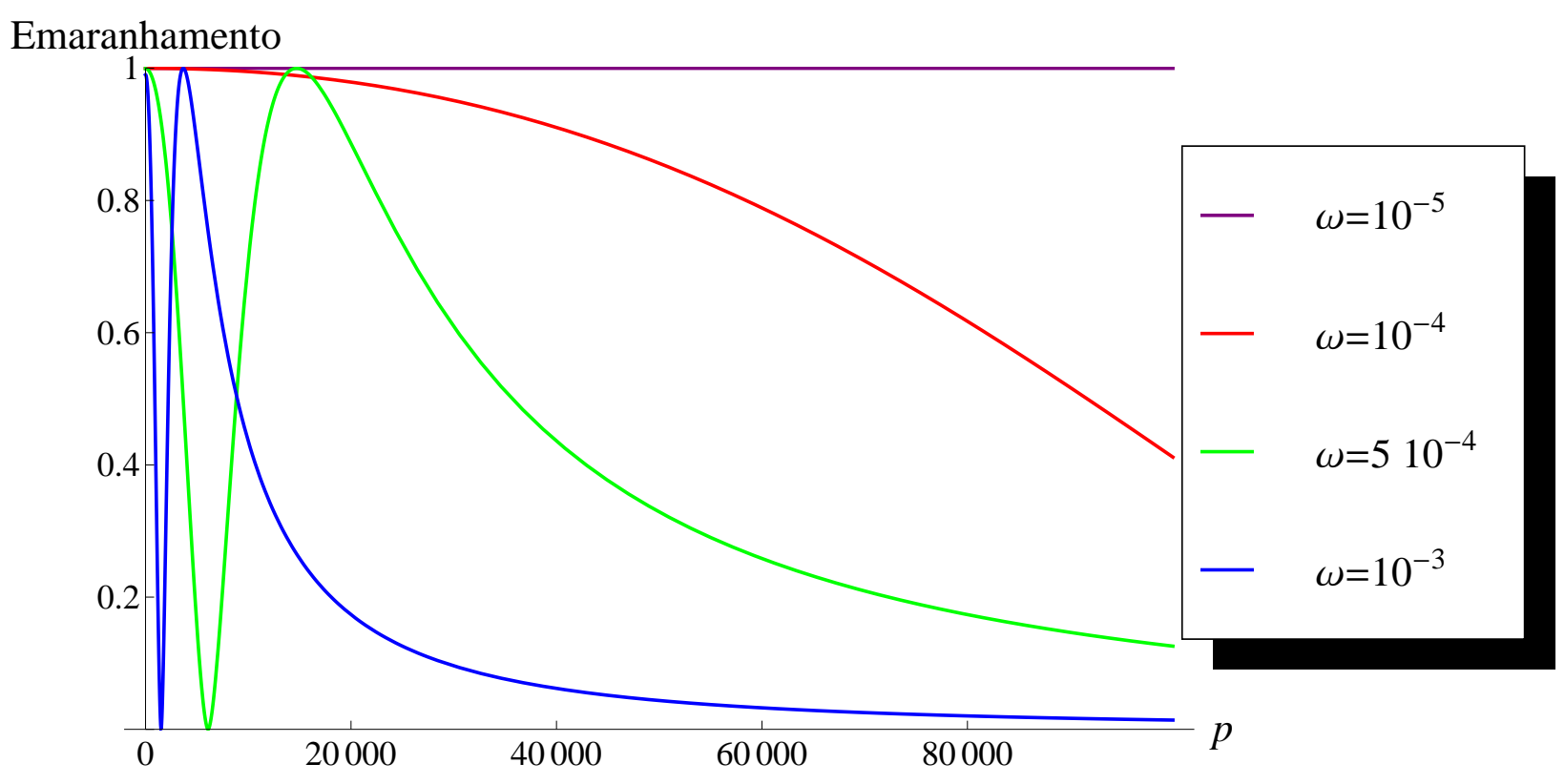

Figure 4.2.1: Esse gráfico mostra a quantidade de emaranhamento do estado $\left|\tilde{\Psi}_{11}\right\rangle$ em função do momento $p$ do elétron para diferentes frequências do campo eletromagnético. Varremos uma região de momento em que o elétron tem de 0 a $0.999999 c$ e consideramos frequências dentro do infravermelho.

onde o estado $\left|\Phi_{11}^{\text {lin }}\right\rangle$ sempre representa um estado maximalmente emaranhado enquanto que o estado $\left|\Phi_{11}^{c i r c}\right\rangle$ apresenta emaranhamento dependendo do momento $p$ do elétron e da energia $\omega$ dos fótons. Na figura 4.2.1 apresentamos a quantidade de emaranhamento do estado $\left|\Phi_{11}^{\text {circ }}\right\rangle$ em função de $p$ para diferentes valores de $\omega$. Usamos a entropia de von Neumann como quantificador de emaranhamento. Notamos dois comportamentos interessantes para a quantidade de emaranhamento: em primeiro lugar, quando o momento do elétron é suficientemente grande, a quantidade de emaranhamento cai monotonicamente a zero. Este fato pode ser entendido com a ideia de que quanto mais momento o elétron possui, menos chance ele terá de interagir com os fótons e emaranhá-los. Em segundo lugar, observamos que quando o elétron tem momento baixo, para uma frequência suficientemente alta do espectro eletromagnético, observamos um efeito de oscilação da quantidade de emaranhamento. 


\section{Capítulo 5}

\section{Conclusão}

Os resultados do nosso trabalho, contidos no capítulo 4, serão sumarizados a seguir.

Supomos inicialmente que o vácuo de quasifótons é igual ao vácuo de fótons, o que é, em primeiro lugar, razoável em baixas energias devido ao fato da constante de estrutura fina ser pequena e, em segundo lugar, é uma aproximação que é usualmente feita quando tratamos a eletrodinâmica quântica perturbativamente.

Primeiramente, na seção 4.1.1, consideramos estados de dois quasifótons de frequências e polarizações distintas e mostramos que na ausência de campo magnético externo não há emaranhamento entre as frequências dos fótons. Em seguida, na seção 4.1.2, consideramos a mesma situação porém agora com um campo magnético externo e mostramos que há emaranhamento entre as polarizações desses fótons. Calculamos a quantidade de emaranhamento utilizando a entropia de von Neumann e mostramos que podemos controlar os parâmetros do sistema para conseguir qualquer quantidade de emaranhamento.

Numa segunda análise, notamos que era possível, no caso monocromático, levar em conta o vácuo de quasifótons exatamente, o que é algo útil pelo fato de nos permitir estudar situações com energias tão altas quanto o desejável.

Na seção 4.2, mostramos que o vácuo exato de quasifótons representa um estado emaranhado quando escrito em termos de fótons de polarização circular, porém representa um estado separável quando escrito em termos de fótons de polarização linear. Explicamos esse fenômeno com o argumento de que escolhas diferentes de observáveis podem transformar estados emaranhados em estados separáveis.

Na seção 4.2.1, partindo do vácuo exato de quasifótons escrito em termos de fótons de polarização circular, estudamos o emaranhamento dos estados de dois quasifótons truncando suas expressões em termos de fótons para levar em conta apenas amplitudes com número de fótons próximo ao número de quasifótons. No caso de dois quasifótons de polarização linear, observamos a presença de máximo emaranhamento, porém esse emaranhamento observado 
é puramente cinemático devido ao fato de estarmos escrevendo quasifótons de polarização linear em termos de fótons de polarização circular. No caso de dois quasifótons de polarização circular identificamos emaranhamento, porém não sobre polarizações mas sobre número de fótons. Notamos que a quantidade de emaranhamento exibe um comportamento curioso: para frequências do espectro eletromagnético suficientemente altas, a saber, acima do infravermelho, a quantidade de emaranhamento sofre oscilações ao variarmos o momento do elétron, levando o sistema de maximalmente emaranhado para não emaranhado.

\section{$5.1 \quad$ Perspectivas}

Uma questão de crucial interesse nesse trabalho e que continua em aberto é a questão da evolução temporal. Dado que dispomos de um conjunto completo de soluções exatas da equação de Dirac, somos capazes, em princípio, de construir a evolução temporal desse sistema e estudar como a quantidade de emaranhamento de um dado estado evolui com o tempo. Esse tipo de análise nos permitiria ser mais precisos sobre que efeitos esperar na variação da quantidade de emaranhamento dos fótons devido à interação com o elétron. 


\section{Apêndice A}

\section{Algumas Definições de Interesse}

\section{A.1 Soma Direta de Espaços de Hilbert}

Sejam $\mathcal{H}_{1}$ e $\mathcal{H}_{2}$ espaços de Hilbert. Dados $x_{1} \in \mathcal{H}_{1}$ e $x_{2} \in \mathcal{H}_{2}$, definimos o objeto $x_{1} \oplus x_{2}$ com as seguintes propriedades:

1. $\alpha\left(x_{1} \oplus x_{2}\right)=\left(\alpha x_{1}\right) \oplus\left(\alpha x_{2}\right), \quad \alpha \in \mathbb{C}, x_{1} \in \mathcal{H}_{1}, x_{2} \in \mathcal{H}_{2}$,

2. $x_{1} \oplus x_{2}+y_{1} \oplus y_{2}=\left(x_{1}+y_{1}\right) \oplus\left(x_{2}+y_{2}\right), \quad x_{1}, y_{1} \in \mathcal{H}_{1}, x_{2}, y_{2} \in \mathcal{H}_{2}$.

Seja $\mathcal{S}$ o conjunto das combinações lineares finitas dos $x_{1} \oplus x_{2}$, com $x_{1} \in \mathcal{H}_{1}$ e $x_{2} \in \mathcal{H}_{2}$. As propriedades acima fazem de $\mathcal{S}$ um espaço vetorial. Introduzindo o seguinte produto interno em $\mathcal{S}$ :

$$
\left\langle x_{1} \oplus x_{2} \mid y_{1} \oplus y_{2}\right\rangle=\left\langle x_{1} \mid y_{1}\right\rangle+\left\langle x_{2} \mid y_{2}\right\rangle
$$

obtemos um espaço vetorial com produto interno, i.e. um espaço pré-hilbertiano, que, após completamento na norma induzida por tal produto, se torna um espaço de Hilbert que é comumente denotado $\mathcal{H}_{1} \oplus \mathcal{H}_{2}$, o espaço soma direta.

\section{A.2 Produto Tensorial de Espaços de Hilbert}

Sejam $\mathcal{H}_{1}$ e $\mathcal{H}_{2}$ espaços de Hilbert. Dados $x_{1} \in \mathcal{H}_{1}$ e $x_{2} \in \mathcal{H}_{2}$, definimos o objeto $x_{1} \otimes x_{2}$ com as seguintes propriedades:

1. $\alpha\left(x_{1} \otimes x_{2}\right)=\left(\alpha x_{1}\right) \otimes x_{2}=x_{1} \otimes\left(\alpha x_{2}\right), \quad \alpha \in \mathbb{C}, x_{1} \in \mathcal{H}_{1}, x_{2} \in \mathcal{H}_{2}$,

2. $\left(x_{1}+y_{1}\right) \otimes x_{2}=x_{1} \otimes x_{2}+y_{1} \otimes x_{2}, \quad x_{1}, y_{1} \in \mathcal{H}_{1}, x_{2} \in \mathcal{H}_{2}$,

3. $x_{1} \otimes\left(x_{2}+y_{2}\right)=x_{1} \otimes x_{2}+x_{1} \otimes y_{2}, \quad x_{1} \in \mathcal{H}_{1}, x_{2}, y_{2} \in \mathcal{H}_{2}$. 
Seja $\mathcal{T}$ o conjunto das combinações lineares finitas dos $x_{1} \otimes x_{2}$, com $x_{1} \in \mathcal{H}_{1}$ e $x_{2} \in \mathcal{H}_{2}$. As propriedades acima fazem de $\mathcal{T}$ um espaço vetorial. Introduzindo o seguinte produto interno em $\mathcal{T}$ :

$$
\left\langle x_{1} \otimes x_{2} \mid y_{1} \otimes y_{2}\right\rangle=\left\langle x_{1} \mid y_{1}\right\rangle\left\langle x_{2} \mid y_{2}\right\rangle
$$

obtemos um espaço vetorial com produto interno, i.e. um espaço pré-hilbertiano, que, após completamento na norma induzida por tal produto, se torna um espaço de Hilbert que é comumente denotado $\mathcal{H}_{1} \otimes \mathcal{H}_{2}$, o espaço produto tensorial.

\section{A.3 Produto Tensorial de Operadores de Espaços de Hil- bert}

Seja $A$ um operador de um espaço de Hilbert $\mathcal{H}_{1}$ e $B$ um operador de um espaço de Hilbert $\mathcal{H}_{2}$. Definimos o produto tensorial de $A \operatorname{com} B$, i.e. $A \otimes B$, como sendo um operador no espaço de Hilbert produto tensorial $\mathcal{H}_{1} \otimes \mathcal{H}_{2}$ que age da seguinte forma:

$$
(A \otimes B)\left(x_{1} \otimes x_{2}\right)=\left(A x_{1}\right) \otimes\left(B x_{2}\right), \quad \forall x_{1} \in \mathcal{H}_{1}, \forall x_{2} \in \mathcal{H}_{2}
$$

Essa definição se generaliza facilmente para o produto tensorial de $n$ operadores.

\section{A.4 Espaço de Fock}

A importância do espaço de Fock vem do fato de que ele fornece uma realização simples para as relações canônicas de comutação e anticomutação. Dado um espaço de Hilbert $\mathcal{H}$ definimos o espaço de Fock como sendo:

$$
\mathcal{F}(\mathcal{H})=\bigoplus_{n \in \mathbb{N}} \mathcal{H}^{\otimes n}
$$

onde $\mathcal{H}^{\otimes n} \equiv \mathcal{H} \underbrace{\otimes \cdots \otimes}_{n \text { vezes }} \mathcal{H}$. Com as construções de soma direta e produto tensorial dadas acima fica claro como um espaço de Fock é construído para se tornar um espaço de Hilbert. 


\section{Apêndice B}

\section{Transformações Canônicas Lineares}

O intuito desse apêndice é apresentar alguns resultados de interesse sobre as transformações canônicas lineares (TCL), como relações de ortogonalidade e de normalização. Considere dois conjuntos de operadores de criação e aniquilação, $\left\{b^{\dagger}, b\right\}$ e $\left\{c^{\dagger}, c\right\}$ que satisfazem relações canônicas de comutação, i.e.:

$$
\begin{aligned}
& {\left[b_{i}, b_{j}^{\dagger}\right]=\delta_{i j},\left[b_{i}, b_{j}\right]=\left[b_{i}^{\dagger}, b_{j}^{\dagger}\right]=0} \\
& {\left[c_{i}, c_{j}^{\dagger}\right]=\delta_{i j},\left[c_{i}, c_{j}\right]=\left[c_{i}^{\dagger}, c_{j}^{\dagger}\right]=0}
\end{aligned}
$$

Usaremos uma notação matricial nesta seção, de forma que, por exemplo, $b$ denotará o vetor de operadores de aniquilação $b_{k}$. Seja a TCL dada por:

$$
\left\{\begin{array}{l}
b=\phi c+\psi c^{\dagger}+\gamma, \\
b^{\dagger}=c^{\dagger} \phi^{\dagger}+c \psi^{\dagger}+\gamma^{*}
\end{array}\right.
$$

Impondo na (B.0.3) a (B.0.1), obtemos:

$$
\begin{aligned}
{\left[b, b^{\dagger}\right]=} & {\left[\phi c+\psi c^{\dagger}+\gamma, c^{\dagger} \phi^{\dagger}+c \psi^{\dagger}+\gamma^{*}\right] } \\
= & {\left[\phi c, c^{\dagger} \phi^{\dagger}\right]+\left[\psi c^{\dagger}, c \psi^{\dagger}\right] } \\
= & \phi_{a b} \phi_{c d}^{\dagger}\left[c_{b}, c_{c}^{\dagger}\right]+\psi_{a b} \psi_{c d}^{\dagger}\left[c_{b}^{\dagger}, c_{c}\right] \\
= & \phi_{a b} \phi_{c d}^{\dagger} \delta_{b c}-\psi_{a b} \psi_{c d}^{\dagger} \delta_{b c} \\
{\left[b, b^{\dagger}\right]=} & \phi \phi^{\dagger}-\psi \psi^{\dagger} \\
& \therefore \phi \phi^{\dagger}-\psi \psi^{\dagger}=1
\end{aligned}
$$




$$
\begin{aligned}
{[b, b]=} & {\left[\phi c+\psi c^{\dagger}+\gamma, \phi c+\psi c^{\dagger}+\gamma\right] } \\
= & {\left[\phi c, \psi c^{\dagger}\right]+\left[\psi c^{\dagger}, \phi c\right] } \\
= & \phi_{a b} \psi_{c d}\left[c_{b}, c_{d}^{\dagger}\right]+\psi_{a b} \phi_{c d}\left[c_{b}^{\dagger}, c_{d}\right] \\
= & \phi_{a b} \psi_{c d} \delta_{b d}-\psi_{a b} \phi_{c d} \delta_{b d} \\
{[b, b]=} & \phi \psi^{t}-\psi \phi^{t} \\
& \therefore \phi \psi^{t}-\psi \phi^{t}=0
\end{aligned}
$$

Observe que usando (B.0.4) e (B.0.5) podemos obter as seguintes expressões:

$$
\begin{aligned}
& \phi^{\dagger} \phi-\psi^{t} \psi^{*}=1, \\
& \phi^{\dagger} \psi-\psi^{t} \phi^{*}=0 .
\end{aligned}
$$

De fato, partindo de (B.0.4),

$$
\phi \phi^{\dagger}-\psi \psi^{\dagger}=1 \Leftrightarrow \phi^{*} \phi^{t}-\psi^{*} \psi^{t}=1
$$

multiplicamos à esquerda por $\psi^{t}$ e obtemos:

$$
\psi^{t} \phi^{*} \phi^{t}-\psi^{t} \psi^{*} \psi^{t}=\psi^{t} \Leftrightarrow \phi^{\dagger} \psi \phi^{t}-\psi^{t} \psi^{*} \psi^{t}=\psi^{t}
$$

Utilizando nessa última expressão a (B.0.5), concluímos que:

$$
\begin{gathered}
\phi^{\dagger} \underbrace{\psi \phi^{t}}_{=\phi \psi^{t}}-\psi^{t} \psi^{*} \psi^{t}=\psi^{t} \Leftrightarrow\left(\phi^{\dagger} \phi-\psi^{t} \psi^{*}\right) \psi^{t}=\psi^{t} \\
\therefore \phi^{\dagger} \phi-\psi^{t} \psi^{*}=1 .
\end{gathered}
$$

Para deduzirmos a (B.0.7), partimos de (B.0.6) e a multiplicamos por $\phi^{\dagger}$ pela direita:

$$
\begin{gathered}
\phi^{\dagger} \underbrace{\phi \phi^{\dagger}}_{=1+\psi \psi^{\dagger}}-\psi^{t} \psi^{*} \phi^{\dagger}=\phi^{\dagger} \\
\Leftrightarrow \phi^{\dagger} \psi \psi^{\dagger}-\psi^{t} \underbrace{\psi^{*} \phi^{\dagger}}_{=\phi^{*} \psi^{\dagger}}=0 \Leftrightarrow\left(\phi^{\dagger} \psi-\psi^{t} \phi^{*}\right) \psi^{\dagger}=0 \\
\therefore \phi^{\dagger} \psi-\psi^{t} \phi^{*}=0 .
\end{gathered}
$$


Tendo em mente as expressões (B.0.4), (B.0.5), (B.0.6) e (B.0.7) é imediato inverter a (B.0.3). De fato:

$$
\left\{\begin{array}{l}
\phi^{\dagger} b=\phi^{\dagger} \phi c+\phi^{\dagger} \psi c^{\dagger}+\phi^{\dagger} \gamma \\
\psi^{t} b^{\dagger}=\psi^{t} c^{\dagger} \phi^{\dagger}+\psi^{t} c \psi^{\dagger}+\psi^{t} \gamma^{*}
\end{array}\right.
$$

Subtraindo uma expressão da outra, obtemos:

$$
\begin{gathered}
\phi^{\dagger} b-\psi^{t} b^{\dagger}=\phi^{\dagger} \phi c-\psi^{t} \underbrace{c \psi^{\dagger}}_{=\psi^{*} c}+\phi^{\dagger} \psi c^{\dagger}-\psi^{t} \underbrace{c^{\dagger} \phi^{\dagger}}_{=\phi^{*} c^{\dagger}}+\phi^{\dagger} \gamma-\psi^{t} \gamma^{*} \\
\Leftrightarrow \phi^{\dagger} b-\psi^{t} b^{\dagger}=\underbrace{\left(\phi^{\dagger} \phi-\psi^{t} \psi^{*}\right)}_{=1} c+\underbrace{\left(\phi^{\dagger} \psi-\psi^{t} \phi^{*}\right)}_{=0} c^{\dagger}+\phi^{\dagger} \gamma-\psi^{t} \gamma^{*} \\
\therefore c=\phi^{\dagger} b-\psi^{t} b^{\dagger}+\psi^{t} \gamma^{*}-\phi^{\dagger} \gamma
\end{gathered}
$$




\section{Apêndice C}

\section{Sistemas com Hamiltonianos Quadráticos}

O intuito desse apêndice é mostrar como podemos usar transformações canônicas lineares para reduzir Hamiltonianos quadráticos a uma forma mais simples. Este texto é baseado no segundo apêndice apresentado em [7]. Seja um Hamiltoniano dado por:

$$
H=b^{\dagger} A b+\frac{1}{2}\left(b B b+b^{\dagger} B^{\dagger} b^{\dagger}\right)+\left(D^{*} b+b^{\dagger} D\right)+H_{0},
$$

onde $\left\{b^{\dagger}, b\right\}$ são operadores de criação e aniquilação que satisfazem as relações canônicas de comutação:

$$
\left[b_{i}, b_{j}^{\dagger}\right]=\delta_{i j},\left[b_{i}, b_{j}\right]=\left[b_{i}^{\dagger}, b_{j}^{\dagger}\right]=0 .
$$

Da hermiticidade de $H$, i.e. $H^{\dagger}=H$, concluímos que $A^{\dagger}=A$ e $H_{0}^{*}=H_{0}$. Pelo fato de $\left[b_{i}, b_{j}\right]=\left[b_{i}^{\dagger}, b_{j}^{\dagger}\right]=0$, concluímos que $B$ deve ser simétrico, i.e. $B=B^{t}$. Realizando a seguinte transformação canônica linear:

$$
\left\{\begin{array}{l}
b=\phi c+\psi c^{\dagger}+\gamma, \\
b^{\dagger}=c^{\dagger} \phi^{\dagger}+c \psi^{\dagger}+\gamma^{*}
\end{array}\right.
$$

obtemos a seguinte Hamiltoniana:

$$
H=c^{\dagger} \bar{A} c+\frac{1}{2}\left(c \bar{B} c+c^{\dagger} \bar{B}^{\dagger} c^{\dagger}\right)+\left(\bar{D}^{*} c+c^{\dagger} \bar{D}\right)+\bar{H}_{0}
$$

Calculemos os termos $\bar{A}, \bar{B}, \bar{D}$ e $\bar{H}_{0}$, em termos de $A, B, D$ e $H_{0}$. 
1. $b^{\dagger} A b$ :

$$
\begin{aligned}
b^{\dagger} A b= & \left(c^{\dagger} \phi^{\dagger}+c \psi^{\dagger}+\gamma^{*}\right) A\left(\phi c+\psi c^{\dagger}+\gamma\right) \\
= & c^{\dagger}\left(\phi^{\dagger} A \phi+\psi^{t} A^{t} \psi^{*}\right) c+c\left(\psi^{\dagger} A \phi\right) c+c^{\dagger}\left(\phi^{\dagger} A \psi\right) c^{\dagger}+ \\
& +\left(\gamma^{*} A \phi+\gamma A^{t} \psi^{*}\right) c+c^{\dagger}\left(\phi^{\dagger} A \gamma+\psi^{t} A^{t} \gamma^{*}\right)+\operatorname{Tr}\left(\psi^{\dagger} A \psi\right)+\gamma^{*} A \gamma
\end{aligned}
$$

2. $b B b$ :

$$
\begin{aligned}
b B b= & \left(\phi c+\psi c^{\dagger}+\gamma\right) B\left(\phi c+\psi c^{\dagger}+\gamma\right) \\
= & c^{\dagger}\left(2 \psi^{t} B \phi\right) c+c\left(\phi^{t} B \phi\right) c+c^{\dagger}\left(\psi^{t} B \psi\right) c^{\dagger}+ \\
& +\left(2 \phi^{t} B \gamma\right) c+c^{\dagger}\left(2 \psi^{t} B \gamma\right)+\operatorname{Tr}\left(\phi^{t} B \psi\right)+\gamma B \gamma
\end{aligned}
$$

3. $b^{\dagger} B^{\dagger} b^{\dagger}:$

$$
\begin{aligned}
b^{\dagger} B^{\dagger} b^{\dagger}= & \left(c^{\dagger} \phi^{\dagger}+c \psi^{\dagger}+\gamma^{*}\right) B^{\dagger}\left(c^{\dagger} \phi^{\dagger}+c \psi^{\dagger}+\gamma^{*}\right) \\
= & c^{\dagger}\left(2 \phi^{\dagger} B^{\dagger} \psi^{*}\right) c+c\left(\psi^{\dagger} B^{\dagger} \psi^{*}\right) c+c^{\dagger}\left(\phi^{\dagger} B^{\dagger} \phi^{*}\right) c^{\dagger}+ \\
& +\left(2 \psi^{\dagger} B^{\dagger} \gamma^{*}\right) c+c^{\dagger}\left(2 \phi^{\dagger} B^{\dagger} \gamma^{*}\right)+\operatorname{Tr}\left(\psi^{\dagger} B^{\dagger} \phi^{*}\right)+\gamma^{*} B^{\dagger} \gamma^{*}
\end{aligned}
$$

4. $D^{*} b+b^{\dagger} D$ :

$$
\begin{aligned}
D^{*} b+b^{\dagger} D & =D^{*}\left(\phi c+\psi c^{\dagger}+\gamma\right)+\left(c^{\dagger} \phi^{\dagger}+c \psi^{\dagger}+\gamma^{*}\right) D \\
& =\left(D^{*} \phi+D \psi^{*}\right) c+c^{\dagger}\left(\psi^{t} D^{*}+\phi^{\dagger} D\right)+D^{*} \gamma+\gamma^{*} D
\end{aligned}
$$

Reunindo os resultados obtidos, concluímos que:

$$
\begin{aligned}
\bar{A}= & \phi^{\dagger}\left(A \phi+B^{\dagger} \psi^{*}\right)+\psi^{t}\left(A^{t} \psi^{*}+B \phi\right) \\
\bar{B}= & 2 \psi^{\dagger} A \phi+\phi^{t} B \phi+\psi^{\dagger} B^{\dagger} \psi^{*} \\
\bar{D}= & \left(\phi^{\dagger} A+\psi^{t} B\right) \gamma+\left(\psi^{t} A^{t}+\phi^{\dagger} B^{\dagger}\right) \gamma^{*}+\psi^{t} D^{*}+\phi^{\dagger} D \\
\bar{H}_{0}= & \gamma^{*} A \gamma+\frac{1}{2} \gamma B \gamma+\frac{1}{2} \gamma^{*} B^{\dagger} \gamma^{*}+D^{*} \gamma+\gamma^{*} D+ \\
& +\frac{1}{2} \operatorname{Tr}\left(2 \psi^{\dagger} A \psi+\phi^{t} B \psi+\psi^{\dagger} B^{\dagger} \phi^{*}\right)+H_{0}
\end{aligned}
$$

Queremos transformar o Hamiltoniano (C.0.2) em:

$$
H=c^{\dagger} r c+\kappa,
$$


onde $r$ é uma matriz não singular e $\kappa$ uma constante. Observando a expressão que obtivemos para $\bar{A}$, se supormos que:

$$
\begin{aligned}
\phi r & =A \phi+B^{\dagger} \psi^{*}, \\
-\psi^{*} r & =A^{t} \psi^{*}+B \phi,
\end{aligned}
$$

então, tendo em mente a relação (B.0.6), concluímos que $\bar{A}=r$. Como o termo $\psi^{\dagger} A \phi$ na expressão para $\bar{B}$ deve ser simétrico, podemos trocá-lo por $\frac{1}{2}\left(\psi^{\dagger} A \phi+\phi^{t} A^{t} \psi^{*}\right)$ e, dessa forma:

$$
\begin{aligned}
\bar{B} & =\underbrace{\left(\psi^{\dagger} A+\phi^{t} B\right)}_{=-r^{t} \psi^{\dagger}} \phi+\underbrace{\left(\phi^{t} A^{t}+\psi^{\dagger} B^{\dagger}\right)}_{=r^{t} \phi^{t}} \psi^{*} \\
& =r^{t}\left(\phi^{t} \psi^{*}-\psi^{\dagger} \phi\right) \\
\bar{B} & =-r^{t}\left(\phi^{\dagger} \psi-\psi^{t} \phi^{*}\right)^{\dagger}
\end{aligned}
$$

i.e., tendo em mente a (B.0.7), $\bar{B}=0$. Analisando o termo $\bar{D}$, percebemos que podemos reescrevê-lo na forma:

$$
\begin{aligned}
\bar{D} & =\phi^{\dagger}\left(A \gamma+B^{\dagger} \gamma^{*}+D\right)+\psi^{t}\left(A^{t} \gamma^{*}+B \gamma+D^{*}\right) \\
& =\phi^{\dagger}\left(A \gamma+B^{\dagger} \gamma^{*}+D\right)+\psi^{t}\left(A \gamma+B^{\dagger} \gamma^{*}+D\right) .^{*}
\end{aligned}
$$

Isso nos diz que se escolhermos $\gamma$ de forma a obedecer a equação

$$
A \gamma+B^{\dagger} \gamma^{*}+D=0
$$

então teremos $\bar{D}=0$. Essas hipóteses nos permitem reescrever a expressão (C.0.2) na forma desejada (C.0.3), onde $\kappa=\bar{H}_{0}$ e $\bar{A}=r$. Notemos, entretanto, que as equações (C.0.4) e (C.0.5) podem não ter solução. De fato, usando a relação (B.0.7), vemos que:

$$
\begin{gathered}
\psi^{\dagger} \phi r=\psi^{\dagger} A \phi+\psi^{\dagger} B^{\dagger} \psi^{*} \\
-\phi^{t} \psi^{*} r=\phi^{t} A^{t} \psi^{*}+\phi^{t} B \phi \\
\Rightarrow\left(\psi^{\dagger} \phi-\phi^{t} \psi^{*}\right) r=\psi^{\dagger} A \phi+\psi^{\dagger} B^{\dagger} \psi^{*}+\phi^{t} A^{t} \psi^{*}+\phi^{t} B \phi \\
\therefore \psi^{\dagger} A \phi+\psi^{\dagger} B^{\dagger} \psi^{*}+\phi^{t} A^{t} \psi^{*}+\phi^{t} B \phi=0,
\end{gathered}
$$


i.e. a relação (C.0.7) deve ser verificada por $\phi$ e $\psi$. Podemos reescrever a (C.0.7) na forma:

$$
\left(\begin{array}{cc}
\phi^{t} & \psi^{\dagger}
\end{array}\right)\left(\begin{array}{cc}
B & A^{t} \\
A & B^{\dagger}
\end{array}\right)\left(\begin{array}{c}
\phi \\
\psi^{*}
\end{array}\right)=0,
$$

o que nos diz que as condições (C.0.4) e (C.0.5) só podem ser satisfeitas se

$$
|\operatorname{det} B|^{2}-(\operatorname{det} A)^{2} \neq 0 \text {. }
$$




\section{Apêndice D}

\section{Representação de Fock-Bargmann}

O intuito deste apêndice é apresentar resultados básicos sobre a representação de FockBargmann. Citamos como referências sobre o assunto os textos [14, 19].

Considere um sistema quântico com $n$ graus de liberdade que seja descrito pelos operadores $\hat{q}_{i}$ e $\hat{p}_{i}$, com $i \in\{1, \ldots, n\}$. Esses operadores satisfazem as relações de comutação:

$$
\begin{gathered}
{\left[\hat{q}_{i}, \hat{q}_{j}\right]=\left[\hat{p}_{i}, \hat{p}_{j}\right]=0,} \\
{\left[\hat{q}_{i}, \hat{p}_{j}\right]=i \delta_{i j} .}
\end{gathered}
$$

Podemos igualmente descrever esse sistema em termos de operadores de criação e aniquilação $\hat{a}$ e $\hat{a}^{\dagger}$ :

$$
\begin{aligned}
\hat{a}_{i} & =\frac{1}{\sqrt{2}}\left(\hat{q}_{i}+i \hat{p}_{i}\right), \\
\hat{a}_{i}^{\dagger} & =\frac{1}{\sqrt{2}}\left(\hat{q}_{i}-i \hat{p}_{i}\right) .
\end{aligned}
$$

As relações de comutação em termos de operadores de criação e aniquilação se escrevem na forma:

$$
\begin{gathered}
{\left[\hat{a}_{i}, \hat{a}_{j}\right]=\left[\hat{a}_{i}^{\dagger}, \hat{a}_{j}^{\dagger}\right]=0,} \\
{\left[\hat{a}_{i}, \hat{a}_{j}^{\dagger}\right]=\delta_{i j} .}
\end{gathered}
$$

Dado esse conjunto de operadores de criação e aniquilação, identificamos $n$ operadores Hermitianos, a saber os operadores $\hat{N}_{i}=\hat{a}_{i}^{\dagger} \hat{a}_{i}$. Seja $|0\rangle$ o estado que é aniquilado por todos os operadores $\hat{a}_{i}$, i.e.:

$$
\hat{a}_{i}|0\rangle=0, \quad \forall i \in\{1, \ldots, n\} .
$$


Usando as relações de comutação podemos criar o espaço de Hilbert dos estados que diagonalizam todos os operadores $\hat{N}_{i}$ simultaneamente que têm a forma:

$$
\left|k_{1}, \ldots, k_{n}\right\rangle=\frac{\left(\hat{a}_{1}^{\dagger}\right)^{k_{1}} \cdots\left(\hat{a}_{n}^{\dagger}\right)^{k_{n}}}{\sqrt{\left(k_{1} !\right) \cdots\left(k_{n} !\right)}}|0\rangle .
$$

O formalismo apresentado brevemente acima surge naturalmente na eletrodinâmica quântica, por exemplo, onde os operadores $\hat{a}_{i}$ e $\hat{a}_{i}^{\dagger}$ são interpretados como operadores de criação e aniquilação de fótons, os operadores $\hat{N}_{i}$ são observáveis associados ao número de fótons do tipo $i$ e $\left|k_{1}, \ldots, k_{n}\right\rangle$ é um estado que apresenta $k_{j}$ fótons do tipo $j, j \in\{1, \ldots n\}$.

Seja $\hat{D}\left(z_{1}, \ldots, z_{n}\right)$ o operador definido da seguinte forma:

$$
\hat{D}\left(z_{1}, \ldots, z_{n}\right)=e^{\sum_{i}\left(z_{i} \hat{a}_{i}^{\dagger}-z_{i}^{*} \hat{a}_{i}^{\dagger}\right)}, z_{i} \in \mathbb{C}
$$

Esse operador é conhecido como operador de deslocamento. Observe que podemos reescrever a (D.0.1) como:

$$
\hat{D}\left(z_{1}, \ldots, z_{n}\right)=\prod_{i} e^{-\left|z_{i}\right|^{2} / 2} e^{z_{i} \hat{a}_{i}^{\dagger}} e^{z_{i}^{*} \hat{a}_{i}}
$$

Definimos o estado $\left|z_{1}, \ldots, z_{n}\right\rangle$ como:

$$
\left|z_{1}, \ldots, z_{n}\right\rangle=\hat{D}\left(z_{1}, \ldots, z_{n}\right)|0\rangle
$$

Usando a (D.0.2) na (D.0.3), chegamos em:

$$
\left|z_{1}, \ldots, z_{n}\right\rangle=\prod_{i} e^{-\left|z_{i}\right|^{2} / 2} \sum_{k_{i}=0}^{\infty} \frac{z_{i}^{k_{i}}}{\sqrt{k_{i} !}}\left|k_{i}\right\rangle .
$$

Os estados $|\boldsymbol{z}\rangle \equiv\left|z_{1}, \ldots, z_{n}\right\rangle$ são usualmente conhecidos como estados coerentes e eles são capazes de mapear o espaço de Hilbert $\mathcal{H}$ gerado por $\left\{\left|k_{1}, \ldots, k_{n}\right\rangle\right\}$ em $\mathbb{C}^{n}$. Listamos algumas propriedades de interesse desses estados:

1. Minimização das relações de incerteza:

$$
\left(\Delta \hat{q}_{i}\right)_{\boldsymbol{z}}\left(\Delta \hat{p}_{i}\right)_{\boldsymbol{z}}=\frac{1}{2}
$$

2. Os estados $|\boldsymbol{z}\rangle$ são autoestados dos operadores $\hat{a}_{i}$ :

$$
\hat{a}_{i}|\boldsymbol{z}\rangle=z_{i}|\boldsymbol{z}\rangle
$$


3. Os estados $|\boldsymbol{z}\rangle$ formam um conjunto supercompleto de estados, i.e.:

$$
\mathbb{I}=\frac{1}{\pi^{n}} \int\left(\prod_{i} d z_{i 1} d z_{i 2}\right)\left|z_{1}, \ldots, z_{n}\right\rangle\left\langle z_{1}, \ldots, z_{n}\right|, \quad z_{i}=z_{i 1}+i z_{i 2} .
$$

Seja $|\psi\rangle \in \mathcal{H}$, tal que:

$$
|\psi\rangle=\sum_{k_{1}, \ldots, k_{n}} c_{k_{1} \ldots k_{n}}\left|k_{1}, \ldots, k_{n}\right\rangle \quad\langle\psi \mid \psi\rangle=\sum_{k_{1}, \ldots, k_{n}}\left|c_{k_{1} \ldots k_{n}}\right|^{2}=1
$$

Fazendo o produto escalar entre $|\psi\rangle$ e os estados $|\boldsymbol{z}\rangle$ definidos pela (D.0.4), temos:

$$
\begin{aligned}
\left\langle z_{1}, \ldots, z_{n} \mid \psi\right\rangle & =e^{-\sum_{i}\left|z_{i}\right|^{2} / 2} \sum_{k_{1}, \ldots, k_{n}} c_{k_{1} \ldots k_{n}} \frac{\left(z_{1}^{*}\right)^{k_{1}} \cdots\left(z_{n}^{*}\right)^{k_{n}}}{\sqrt{k_{1} ! \cdots k_{n} !}} \\
& \equiv e^{-\sum_{i}\left|z_{i}\right|^{2} / 2} \psi\left(\boldsymbol{z}^{*}\right)
\end{aligned}
$$

onde definimos:

$$
\begin{gathered}
\psi(\boldsymbol{z})=\sum_{k_{1}, \ldots, k_{n}} c_{k_{1}, \ldots, k_{n}} u_{k_{1}, \ldots, k_{n}}(\boldsymbol{z}), \\
u_{k_{1}, \ldots, k_{n}}(\boldsymbol{z})=\frac{z_{1}^{k_{1}} \cdots z_{n}^{k_{n}}}{\sqrt{k_{1} ! \cdots k_{n} !}} .
\end{gathered}
$$

Como $\sum_{k_{1}, \ldots, k_{n}}\left|c_{k_{1} \ldots k_{n}}\right|^{2}=1$, então a série presente em (D.0.5) converge uniformemente em qualquer compacto de $\mathbb{C}^{n}$, o que implica que $\psi(\boldsymbol{z})$ é uma função analítica em $\mathbb{C}^{n} \mathrm{e}$

$$
\begin{aligned}
\|\psi(\boldsymbol{z})\|^{2} & =\langle\psi \mid \psi\rangle=\frac{1}{\pi^{n}} \int\left(\prod_{i} d z_{i 1} d z_{i 2}\right)\left|\left\langle z_{1}, \ldots, z_{n} \mid \psi\right\rangle\right|^{2} \\
& =\int\left(\frac{1}{\pi^{n}} \prod_{i} e^{\left|z_{i}\right|^{2}} d z_{i 1} d z_{i 2}\right)|\psi(\boldsymbol{z})|^{2} \\
\|\psi(\boldsymbol{z})\|^{2} & \equiv \int d \mu(\boldsymbol{z})|\psi(\boldsymbol{z})|^{2}<\infty,
\end{aligned}
$$

onde $d \mu(\boldsymbol{z})=\frac{1}{\pi^{n}} \prod_{i} e^{\left|z_{i}\right|^{2}} d z_{i 1} d z_{i 2}$. O conjunto das funções analíticas em $\mathbb{C}^{n}$, satisfazendo a condição (D.0.6) e munido do produto interno

$$
\left\langle\psi_{1}(\boldsymbol{z}) \mid \psi_{2}(\boldsymbol{z})\right\rangle=\int d \mu(\boldsymbol{z}) \psi_{1}^{*}(\boldsymbol{z}) \psi_{2}(\boldsymbol{z})
$$

segundo Bargmann [11], forma um espaço de Hilbert. Dessa forma, temos uma realização 
concreta do espaço de Hilbert $\mathcal{H}$ num espaço de funções analíticas em $\mathbb{C}^{n}$. Essa representação é comumente denominada representação de Fock-Bargmann. Nessa representação os operadores $\hat{a}_{i}$ e $\hat{a}_{i}^{\dagger}$ agem respectivamente como uma derivada e como um operador multiplicativo, i.e.:

$$
\begin{aligned}
& \hat{a}_{i} \rightarrow \frac{\partial}{\partial z_{i}}, \\
& \hat{a}_{i}^{\dagger} \rightarrow z_{i} .
\end{aligned}
$$

Observe que se $|\psi\rangle$ representa um estado não emaranhado, i.e.:

$$
|\psi\rangle=\left|\psi_{1}\right\rangle \otimes \cdots \otimes\left|\psi_{n}\right\rangle, \quad\left|\psi_{j}\right\rangle=\sum_{k_{j}=0}^{\infty} c_{k_{j}}\left|k_{j}\right\rangle
$$

então, na representação de Fock-Bargmann, a função $\psi(\boldsymbol{z})$ será da forma:

$$
\begin{aligned}
\psi(\boldsymbol{z}) & =\sum_{k_{1}, \ldots, k_{n}} c_{k_{1}} \ldots c_{k_{n}} u_{k_{1} \ldots k_{n}}(\boldsymbol{z}) \\
& =\prod_{i=1}^{n}\left(\sum_{k_{i}=0}^{\infty} c_{k_{i}} u_{k_{i}}\left(z_{i}\right)\right) \\
\psi(\boldsymbol{z}) & =\psi_{1}\left(z_{1}\right) \cdots \psi_{n}\left(z_{n}\right),
\end{aligned}
$$

onde $\psi_{j}\left(z_{j}\right)=\sum_{k_{j}} c_{k_{j}} u_{k_{j}}\left(z_{j}\right)$. Isso nos diz que se um estado é separável, então o seu representante na representação de Fock-Bargmann é uma função $\psi(\boldsymbol{z})$ que se fatoriza num produto como em (D.0.7). 


\section{Apêndice E}

\section{Um Fato Sobre Estados Comprimidos}

\section{E.1 Fatoração do Operador de Compressão}

Seja

$$
S(\xi)=\exp \left[\frac{1}{2}\left(\xi\left(\hat{a}^{\dagger}\right)^{2}-\xi^{*} \hat{a}^{2}\right)\right]
$$

o operador de compressão. Gostaríamos de mostrar que tal operador pode ser fatorado na forma

$$
S(\xi)=e^{\alpha\left(\hat{a}^{\dagger}\right)^{2}} e^{\beta \hat{a}^{2}} e^{\gamma\left[\hat{a}^{2},\left(\hat{a}^{\dagger}\right)^{2}\right]} .
$$

Para demonstrarmos (E.1.1) vamos considerar $X=\left(\hat{a}^{\dagger}\right)^{2}, Y=\hat{a}^{2}$ e $Z=\left[\hat{a}^{2},\left(\hat{a}^{\dagger}\right)^{2}\right]=$ $2\left(2 \hat{a}^{\dagger} \hat{a}+1\right)$, o que nos leva a concluir que

$$
[X, Z]=-8 X \quad \text { e } \quad[Y, Z]=8 Y
$$

Além disso, definimos

$$
U(\tau)=\exp \left[\frac{\tau}{2}\left(\xi X-\xi^{*} Y\right)\right]=e^{\alpha(\tau) X} e^{\beta(\tau) Y} e^{\gamma(\tau) Z} .
$$

Derivando $U$ em relação a $\tau$, temos:

$$
\begin{aligned}
\frac{d U}{d \tau}=\frac{1}{2}(\xi X & \left.-\xi^{*} Y\right) U=\alpha^{\prime} X U+\beta^{\prime} e^{\alpha(\tau) X} Y e^{\beta(\tau) Y} e^{\gamma(\tau) Z}+\gamma^{\prime} e^{\alpha(\tau) X} e^{\beta(\tau) Y} Z e^{\gamma(\tau) Z} \\
e^{\alpha X} Y e^{-\alpha X} & =Y+[\alpha X, Y]+\frac{1}{2 !}[\alpha X,[\alpha X, Y]]+\frac{1}{3 !}[\alpha X,[\alpha X,[\alpha X, Y]]]+\cdots \\
& =Y+\alpha Z-4 \alpha^{2} X
\end{aligned}
$$




$$
\begin{aligned}
& e^{\beta Y} Z e^{-\beta Y}=Z+[\beta Y, Z]+\frac{1}{2 !}[\beta Y,[\beta Y, Z]]+\cdots \\
& =Z+8 \beta Y \\
& e^{\alpha X} Z e^{-\alpha X}=Z+[\alpha X, Z]+\frac{1}{2 !}[\alpha X,[\alpha X, Z]]+\cdots \\
& =Z-8 \alpha X \\
& e^{\alpha X}(Z+8 \beta Y) e^{-\alpha X}=e^{\alpha X} Z e^{-\alpha X}+8 \beta e^{\alpha X} Y e^{-\alpha X} \\
& =(Z-8 \alpha X)+8 \beta\left(Y+\alpha Z-4 \alpha^{2} X\right) \\
& =\left(-8 \alpha-32 \alpha^{2} \beta\right) X+8 \beta Y+(1+8 \alpha \beta) Z \\
& \therefore \frac{\xi}{2} X-\frac{\xi^{*}}{2} Y=\alpha^{\prime} X+\beta^{\prime}\left(Y+\alpha Z-4 \alpha^{2} X\right)+\gamma^{\prime}\left[\left(-8 \alpha-32 \alpha^{2} \beta\right) X+8 \beta Y+(1+8 \alpha \beta) Z\right] \\
& \left\{\begin{array}{l}
\alpha^{\prime}-4 \alpha^{2} \beta^{\prime}-8 \gamma^{\prime} \alpha(1+4 \alpha \beta)=\frac{\xi}{2} \\
\beta^{\prime}+8 \gamma^{\prime} \beta=-\frac{\xi^{*}}{2} \\
\beta^{\prime} \alpha+\gamma^{\prime}(1+8 \alpha \beta)=0
\end{array}\right.
\end{aligned}
$$

Resolvendo esse sistema, obtemos:

$$
\begin{aligned}
& \alpha(\tau)=\frac{1}{2}\left(\frac{\xi}{\xi^{*}}\right)^{1 / 2} \tan (\tau|\xi|), \\
& \beta(\tau)=-\frac{1}{2}\left(\frac{\xi}{\xi^{*}}\right)^{-1 / 2} \cos (\tau|\xi|) \sin (\tau|\xi|), \\
& \gamma(\tau)=-\frac{1}{4} \ln [\cos (\tau|\xi|)] .
\end{aligned}
$$

\section{Portanto}

$$
S(\xi)=\exp \left[\frac{1}{2}\left(\xi\left(\hat{a}^{\dagger}\right)^{2}-\xi^{*} \hat{a}^{2}\right)\right]=\frac{1}{\sqrt{\cos (r)}} e^{\frac{1}{2} e^{i \theta} \tan (r)\left(\hat{a}^{\dagger}\right)^{2}} e^{-\frac{1}{2} e^{-i \theta} \cos (r) \sin (r) \hat{a}^{2}} e^{-\ln [\cos (r)] \hat{a}^{\dagger} \hat{a}}
$$




\section{Apêndice F}

\section{O Vácuo de Quasifótons em Termos dos Operadores de Compressão de duas Polarizações e de Deslocamento}

Considere o estado $|\boldsymbol{z}, \xi\rangle, \boldsymbol{z}=\left(z_{1}, z_{2}\right) \in \mathbb{C}^{2}$, definido por:

$$
|\boldsymbol{z}, \xi\rangle=S_{12}(\xi) D(\boldsymbol{z})|0\rangle,
$$

onde

$$
S_{12}(\xi)=\exp \left[\xi \hat{a}_{1}^{\dagger} \hat{a}_{2}^{\dagger}-\xi^{*} \hat{a}_{1} \hat{a}_{2}\right] \quad \text { e } \quad D(\boldsymbol{z})=D\left(z_{1}\right) D\left(z_{2}\right), \quad D\left(z_{i}\right)=\exp \left[z_{i} \hat{a}_{i}^{\dagger}-z_{i}^{*} \hat{a}_{i}\right] .
$$

Mostraremos que o estado $|\boldsymbol{\alpha}, \xi\rangle$ pode ser escrito na forma

$$
|\boldsymbol{z}, \xi\rangle=k \exp \left[A \hat{a}_{1}^{\dagger} \hat{a}_{2}^{\dagger}+B_{1} \hat{a}_{1}^{\dagger}+B_{2} \hat{a}_{2}^{\dagger}\right]|0\rangle,
$$

onde $k$ é uma constante de normalização.

Para começarmos a demonstração, observe que:

$$
\begin{aligned}
D\left(z_{i}\right) & =e^{z_{i} \hat{a}_{i}^{\dagger}} e^{-z_{i}^{*} \hat{a}_{i}} e^{\frac{1}{2}\left[z_{i} \hat{a}_{i}^{\dagger}, z_{i}^{*} \hat{a}_{i}\right]} \\
& =e^{z_{i} \hat{a}_{i}^{\dagger}} e^{-z_{i}^{*} \hat{a}_{i}} e^{\frac{1}{2}\left|z_{i}\right|^{2}},
\end{aligned}
$$

onde usamos o fato de que se dois operadores $X$ e $Y$ não comutam, i.e. $[X, Y] \neq 0$, porém $[X,[X, Y]]=[Y,[X, Y]]=0$, então

$$
e^{X+Y}=e^{X} e^{Y} e^{-\frac{1}{2}[X, Y]} .
$$


Daqui pra frente vamos ignorar todo termo que seja apenas a exponencial de um número, como o termo $e^{\frac{1}{2}\left|z_{i}\right|^{2}}$ que aparece em $D\left(z_{i}\right)$, pois eles podem ser absorvidos na constante $k$. Note agora que

$$
\begin{aligned}
|\boldsymbol{z}, \xi\rangle & =S_{12}(\xi) D\left(z_{1}\right) D\left(z_{2}\right)|0\rangle \\
& =k S_{12}(\xi) e^{z_{1} \hat{a}_{1}^{\dagger}} e^{-z_{1}^{*} \hat{a}_{1}} e^{z_{2} \hat{a}_{2}^{\dagger}} e^{-z_{2}^{*} \hat{a}_{2}}|0\rangle \\
& =k S_{12}(\xi) e^{z_{1} \hat{a}_{1}^{\dagger}} e^{z_{2} \hat{a}_{2}^{\dagger}} e^{-z_{1}^{*} \hat{a}_{1}} e^{-z_{2}^{*} \hat{a}_{2}}|0\rangle \\
|\boldsymbol{z}, \xi\rangle & =k S_{12}(\xi) e^{z_{1} \hat{a}_{1}^{\dagger}} e^{\alpha z_{2} \hat{a}_{2}^{\dagger}}|0\rangle,
\end{aligned}
$$

pois $e^{-z_{1}^{*} \hat{a}_{1}} e^{-z_{2}^{*} \hat{a}_{2}}|0\rangle=|0\rangle$. Agora vamos nos concentrar em mostrar que o operador $S_{12}(\xi)$ pode ser escrito na forma:

$$
S_{12}(\xi)=\exp \left[\xi \hat{a}_{1}^{\dagger} \hat{a}_{2}^{\dagger}-\xi^{*} \hat{a}_{1} \hat{a}_{2}\right]=e^{\alpha \hat{a}_{1}^{\dagger} \hat{a}_{2}^{\dagger}} e^{\beta \hat{a}_{1} \hat{a}_{2}} e^{\gamma\left[\hat{a}_{1}^{\dagger} \hat{a}_{2}^{\dagger}, \hat{a}_{1} \hat{a}_{2}\right]}
$$

onde

$$
\begin{aligned}
\alpha & =\left(\frac{\xi}{\xi^{*}}\right)^{1 / 2} \tanh |\xi|, \\
\beta & =-\left(\frac{\xi}{\xi^{*}}\right)^{-1 / 2} \cosh |\xi| \sinh |\xi|, \\
\gamma & =\ln (\cosh |\xi|) .
\end{aligned}
$$

Para demonstrar esse fato, seja

$$
U(\tau)=\exp \left[\tau\left(\xi X-\xi^{*} Y\right)\right]=e^{\alpha(\tau) X} e^{\beta(\tau) Y} e^{\gamma(\tau) Z}
$$

onde $X=\hat{a}_{1}^{\dagger} \hat{a}_{2}^{\dagger}, Y=\hat{a}_{1} \hat{a}_{2}, Z=\left[\hat{a}_{1}^{\dagger} \hat{a}_{2}^{\dagger}, \hat{a}_{1} \hat{a}_{2}\right]=-\left(\hat{a}_{1}^{\dagger} \hat{a}_{1}+\hat{a}_{2}^{\dagger} \hat{a}_{2}+1\right)$ e $\alpha(0)=\beta(0)=\gamma(0)=$ 0 . Derivando $U$ em relação a $\tau$, temos, por um lado:

$$
\frac{d U}{d \tau}=\left(\xi X-\xi^{*} Y\right) U
$$

Porém, por outro lado:

$$
\frac{d U}{d \tau}=\alpha^{\prime} X U+\beta^{\prime} e^{\alpha X} Y e^{\beta Y} e^{\gamma Z}+\gamma^{\prime} e^{\alpha X} e^{\beta Y} Z e^{\gamma Z}
$$

Usando o fato de que

$$
e^{X} Y e^{-X}=Y+[X, Y]+\frac{1}{2 !}[X,[X, Y]]+\frac{1}{3 !}[X,[X,[X, Y]]]+\cdots
$$


obtemos

$$
\begin{aligned}
e^{\alpha X} Y & =\left(Y+\alpha Z+\alpha^{2} X\right) e^{\alpha X}, \\
e^{\beta Y} Z & =(Z-2 \beta Y) e^{\beta Y}, \\
e^{\alpha X}(Z-2 \beta Y) & =[2 \alpha(1-\alpha \beta) X-2 \beta Y+(1-2 \alpha \beta) Z] e^{\alpha X} .
\end{aligned}
$$

Igualando as expressões (F.0.3) e (F.0.4), obtemos:

$$
\left[\alpha^{\prime}+\alpha^{2} \beta^{\prime}+2 \alpha(1-\alpha \beta) \gamma^{\prime}-\xi\right] X+\left[\beta^{\prime}-2 \beta \gamma^{\prime}+\xi^{*}\right] Y+\left[\alpha \beta^{\prime}+(1-2 \alpha \beta) \gamma^{\prime}\right] Z=0, \forall \tau
$$

Tal expressão só pode ser identicamente nula se os coeficientes de $X, Y$ e $Z$ se anularem, o que nos fornece um sistema de três equações diferenciais:

$$
\left\{\begin{array}{l}
\alpha^{\prime}+\alpha^{2} \beta^{\prime}+2 \alpha(1-\alpha \beta) \gamma^{\prime}-\xi=0 \\
\beta^{\prime}-2 \beta \gamma^{\prime}+\xi^{*}=0 \\
\alpha \beta^{\prime}+(1-2 \alpha \beta) \gamma^{\prime}=0
\end{array} .\right.
$$

Resolvendo o sistema (F.0.5) com as condições iniciais $\alpha(0)=\beta(0)=\gamma(0)=0$, chegamos em:

$$
\begin{aligned}
\alpha(\tau) & =\left(\frac{\xi}{\xi^{*}}\right)^{1 / 2} \tanh (|\xi| \tau), \\
\beta(\tau) & =-\left(\frac{\xi}{\xi^{*}}\right)^{-1 / 2} \cosh (|\xi| \tau) \sinh (|\xi| \tau), \\
\gamma(\tau) & =\ln (\cosh (|\xi| \tau)) .
\end{aligned}
$$

Tomando $\tau=1$ obtemos a decomposição proposta em (F.0.2). Até o presente momento sabemos que

$$
|\boldsymbol{z}, \xi\rangle=k e^{\alpha \hat{a}_{1}^{\dagger} \hat{a}_{2}^{\dagger}} e^{\beta \hat{a}_{1} \hat{a}_{2}} e^{-\gamma \hat{a}_{1}^{\dagger} \hat{a}_{1}} e^{z_{1} \hat{a}_{1}^{\dagger}} e^{-\gamma \hat{a}_{2}^{\dagger} \hat{a}_{2}} e^{z_{2} \hat{a}_{2}^{\dagger}}|0\rangle
$$

Gostaríamos de comutar as exponenciais $e^{-\gamma \hat{a}_{i}^{\dagger} \hat{a}_{i}}$ e $e^{z_{i} \hat{a}_{i}^{\dagger}}$. Para tanto, utilizaremos a mesma técnica que utilizamos para fatorar o operador $S_{12}(\xi)$, i.e. suporemos que

$$
U(\tau)=e^{-\tau \gamma \hat{a}_{i}^{\dagger} \hat{a}_{i}} e^{\tau z_{i} \hat{a}_{i}^{\dagger}}=e^{\delta(\tau) \hat{a}_{i}^{\dagger}} e^{\epsilon(\tau) \hat{a}_{i}^{\dagger} \hat{a}_{i}}
$$

onde $\delta(0)=\epsilon(0)=0$. Derivando a expressão (F.0.6) em relação a $\tau$, obtemos:

$$
-\gamma \hat{a}_{i}^{\dagger} \hat{a}_{i} U+z_{i} e^{-\tau \gamma \hat{a}_{i}^{\dagger} \hat{a}_{i}} \hat{a}_{i}^{\dagger} e^{\tau z_{i} \hat{a}_{i}^{\dagger}}=\delta^{\prime} \hat{a}_{i}^{\dagger} U+\epsilon^{\prime} \hat{a}_{i}^{\dagger} e^{\delta \hat{a}_{i}^{\dagger}} \hat{a}_{i} e^{\epsilon \hat{a}_{i}^{\dagger} \hat{a}_{i}}
$$


Note que

$$
\begin{aligned}
e^{-\tau \gamma \hat{a}_{i}^{\dagger} \hat{a}_{i}} \hat{a}_{i}^{\dagger} & =e^{-\tau \gamma} \hat{a}_{i}^{\dagger} e^{-\tau \gamma \hat{a}_{i}^{\dagger} \hat{a}_{i}} \\
e^{\delta \hat{a}_{i}^{\dagger}} \hat{a}_{i} & =\left(\hat{a}_{i}-\delta\right) e^{\delta \hat{a}_{i}^{\dagger}}
\end{aligned}
$$

Reunindo nossos resultados, temos

$$
\left(\gamma+\epsilon^{\prime}\right) \hat{a}_{i}^{\dagger} \hat{a}_{i}+\left(z_{i} e^{-\tau \gamma}-\delta^{\prime}+\epsilon^{\prime} \delta\right) \hat{a}_{i}^{\dagger}=0
$$

o que nos dá o seguinte sistema de equações:

$$
\left\{\begin{array}{l}
\epsilon^{\prime}=-\gamma \\
\delta^{\prime}+\gamma \delta-z_{i} e^{-\tau \gamma}=0
\end{array}\right.
$$

cujas soluções que satisfazem as condições $\delta(0)=\epsilon(0)=0$ são:

$$
\begin{aligned}
\epsilon(\tau) & =-\gamma \tau, \\
\delta(\tau) & =z_{i} \tau e^{-\gamma \tau} .
\end{aligned}
$$

Dessa forma, temos que

$$
e^{-\gamma \hat{a}_{i}^{\dagger} \hat{a}_{i}} e^{z_{i} \hat{a}_{i}^{\dagger}}=e^{z_{i} e^{-\gamma} \hat{a}_{i}^{\dagger}} e^{-\gamma \hat{a}_{i}^{\dagger} \hat{a}_{i}}
$$

e

$$
\begin{aligned}
|\boldsymbol{z}, \xi\rangle & =k e^{\alpha \hat{a}_{1}^{\dagger} \hat{a}_{2}^{\dagger}} e^{\beta \hat{a}_{1} \hat{a}_{2}} e^{z_{1} e^{-\gamma} \hat{a}_{1}^{\dagger}} e^{-\gamma \hat{a}_{1}^{\dagger} \hat{a}_{1}} e^{z_{2} e^{-\gamma} \hat{a}_{2}^{\dagger}} e^{-\gamma \hat{a}_{2}^{\dagger} \hat{a}_{2}}|0\rangle \\
& =k e^{\alpha \hat{a}_{1}^{\dagger} \hat{a}_{2}^{\dagger}} e^{\beta \hat{a}_{1} \hat{a}_{2}} e^{z_{1} e^{-\gamma}} \hat{a}_{1}^{\dagger} e^{z_{2} e^{-\gamma} \hat{a}_{2}^{\dagger}} e^{-\gamma \hat{a}_{1}^{\dagger} \hat{a}_{1}} e^{-\gamma \hat{a}_{2}^{\dagger} \hat{a}_{2}}|0\rangle \\
|\boldsymbol{z}, \xi\rangle & =k e^{\alpha \hat{a}_{1}^{\dagger} \hat{a}_{2}^{\dagger}} e^{\beta \hat{a}_{1} \hat{a}_{2}} e^{z_{1} e^{-\gamma}} \hat{a}_{1}^{\dagger} e^{z_{2} e^{-\gamma} \hat{a}_{2}^{\dagger}}|0\rangle .
\end{aligned}
$$

Nosso último passo será comutar os termos $e^{\beta \hat{a}_{1} \hat{a}_{2}}$ e $e^{z_{i} e^{-\gamma} \hat{a}_{i}^{\dagger}}$, onde $i=1,2$. É útil notar que, sendo $X=\beta \hat{a}_{1} \hat{a}_{2}$ e $Y=z_{i} e^{-\gamma} \hat{a}_{i}^{\dagger}$, então claramente $[X, Y]=z_{i} \beta e^{-\gamma} \hat{a}_{j} \neq 0$, onde se $i=1$, então $j=2$ e vice-versa. Além disso, $[X,[X, Y]]=[Y,[X, Y]]=0$. Esses fatos nos permitem fazer uso da expressão (F.0.1). Observe que se a (F.0.1) vale, então:

$$
\begin{array}{r}
e^{X+Y}=e^{X} e^{Y} e^{-\frac{1}{2}[X, Y]} \\
=e^{Y} e^{X} e^{\frac{1}{2}[X, Y]} \\
\therefore e^{X} e^{Y}=e^{Y} e^{X} e^{[X, Y]} .
\end{array}
$$


Assim,

$$
e^{\beta \hat{a}_{1} \hat{a}_{2}} e^{z_{1} e^{-\gamma} \hat{a}_{1}^{\dagger}}=e^{z_{1} e^{-\gamma} \hat{a}_{1}^{\dagger}} e^{\beta \hat{a}_{1} \hat{a}_{2}} e^{z_{1} \beta e^{-\gamma} \hat{a}_{2}}
$$

e

$$
e^{\beta \hat{a}_{1} \hat{a}_{2}} e^{z_{2} e^{-\gamma} \hat{a}_{2}^{\dagger}}=e^{z_{2} e^{-\gamma} \hat{a}_{2}^{\dagger}} e^{\beta \hat{a}_{1} \hat{a}_{2}} e^{z_{2} \beta e^{-\gamma} \hat{a}_{1}}
$$

Finalmente, obtemos:

$$
\begin{aligned}
|\boldsymbol{z}, \xi\rangle & =k e^{\alpha \hat{a}_{1}^{\dagger} \hat{a}_{2}^{\dagger}} e^{\beta \hat{a}_{1} \hat{a}_{2}} e^{z_{1} e^{-\gamma} \hat{a}_{1}^{\dagger}} e^{z_{2} e^{-\gamma} \hat{a}_{2}^{\dagger}}|0\rangle \\
& =k e^{\alpha \hat{a}_{1}^{\dagger} \hat{a}_{2}^{\dagger}} e^{z_{1} e^{-\gamma} \hat{a}_{1}^{\dagger}} e^{\beta \hat{a}_{1} \hat{a}_{2}} e^{z_{1} \beta e^{-\gamma} \hat{a}_{2}} e^{z_{2} e^{-\gamma} \hat{a}_{2}^{\dagger}}|0\rangle \\
& =k e^{\alpha \hat{a}_{1}^{\dagger} \hat{a}_{2}^{\dagger}} e^{z_{1} e^{-\gamma} \hat{a}_{1}^{\dagger}} e^{z_{1} \beta e^{-\gamma} \hat{a}_{2}} e^{\beta \hat{a}_{1} \hat{a}_{2}} e^{z_{2} e^{-\gamma} \hat{a}_{2}^{\dagger}}|0\rangle \\
& =k e^{\alpha \hat{a}_{1}^{\dagger} \hat{a}_{2}^{\dagger}} e^{z_{1} e^{-\gamma} \hat{a}_{1}^{\dagger}} e^{z_{1} \beta e^{-\gamma} \hat{a}_{2}} e^{z_{2} e^{-\gamma} \hat{a}_{2}^{\dagger}} e^{\beta \hat{a}_{1} \hat{a}_{2}} e^{z_{2} \beta e^{-\gamma} \hat{a}_{1}}|0\rangle \\
|\boldsymbol{z}, \xi\rangle & =k e^{\alpha \hat{a}_{1}^{\dagger} \hat{a}_{2}^{\dagger}} e^{z_{1} e^{-\gamma} \hat{a}_{1}^{\dagger}} e^{z_{2} e^{-\gamma} \hat{a}_{2}^{\dagger}}|0\rangle .
\end{aligned}
$$

Observe que comutamos os termos $e^{z_{1} \beta e^{-\gamma} \hat{a}_{2}}$ e $e^{z_{2} e^{-\gamma} \hat{a}_{2}^{\dagger}}$, no entanto, o termo adicional que ganhamos ao fazer essa operação foi absorvido na constante $k$. Concluímos que o estado $|\boldsymbol{z}, \xi\rangle$ é da forma:

$$
\begin{aligned}
|\boldsymbol{z}, \xi\rangle=k \exp \left[e^{i \theta} \tanh (r) \hat{a}_{1}^{\dagger} \hat{a}_{2}^{\dagger}\right. & \left.+\frac{1}{\cosh (r)}\left(z_{1} \hat{a}_{1}^{\dagger}+z_{2} \hat{a}_{2}^{\dagger}\right)\right]|0\rangle, \quad \xi=r e^{i \theta}, \\
A & =e^{i \theta} \tanh (r), \\
B_{1} & =\frac{z_{1}}{\cosh (r)} \\
B_{2} & =\frac{z_{2}}{\cosh (r)} .
\end{aligned}
$$




\section{Referências Bibliográficas}

[1] V. G. Bagrov, P. V. Bozrikov, and D. M. Gitman. A charge in a quantized field of a plane wave. Radiophysics and Quantum Electronics. Radiophysics and Quantum Electronics, 16(1), 1973.

[2] V. G. Bagrov, P. V. Bozrikov, and D. M. Gitman. Electron in The Field of a Plane Quantized Electromagnetic Wave. Theoretical and Mathematical Physics, 14(2), 1973.

[3] V. G. Bagrov, P. V. Bozrikov, D. M. Gitman, and M. M. Lavrov. Electron in the field of a plane quantized monochromatic electromagnetic wave. Russian Physics Journal, 16(8), 1973.

[4] V. G. Bagrov, D. M. Gitman, V. A. Kuchin, and M. M. Lavrov. Fundamental problems of the electrodynamics of electrons in the quantized field of a plane wave I. Russian Physics Journal, 17(12), 1974.

[5] V. G. Bagrov, D. M. Gitman, V. A. Kuchin, and M. M. Lavrov. Fundamental problems of the electrodynamics of electrons in the quantized field of a plane wave II. Russian Physics Journal, 18(7), 1974.

[6] V. G. Bagrov, D. M. Gitman, and M. M. Lavrov. The electron in the quantum field of a plane wave and the classical field of Redmond configuration. Soviet Physics Journal, 17(6), 1974.

[7] V.G. Bagrov and D.M. Gitman. Exact Solutions of Relativistic Wave Equations. Kluber Academic Publishers, 1990.

[8] M. C. Baldiotti and D. M. Gitman. Four-level systems and a universal quantum gate. Arxiv preprint arXiv:0803.0299, page 12, October 2007.

[9] J. C. A. Barata and M. S. Hussein. The Moore-Penrose Pseudoinverse . A Tutorial Review of the Theory Introduction, Motivation and Notation. Organization, (3):1-23. 
[10] F. A. S. Barbosa, A. S. Coelho, A. J. De Faria, K. N. Cassemiro, A. S. Villar, and P. Nussenzveig. Robustness of bipartite Gaussian entangled beams propagating in lossy channels. October, 4(October):7-10, 2010.

[11] V. Bargmann. Remarks on a Hilbert Space of Analytic Functions. Commun Pure Appl Math, 14:187, 1961.

[12] C. H. Bennett, S. Popescu, and B. Schumacher. Concentrating partial entanglement by local operations. Physical Review A, 53(4):2046-2052, April 1996.

[13] R. P. Feynman. Simulating physics with computers. International Journal of Theoretical Physics, 21(6-7):467-488, June 1982.

[14] J.-P. Gazeau. Coherent states in quantum physics, volume 4. Wiley-VCH, 2009.

[15] K Gottfried and T.-M. Yan. Quantum Mechanics: Fundamentals. Springer, second edition, 2004.

[16] S. Haroche and P. Raymond. Cavity Quantum Electrodynamics. Academic Press, New York, 1994.

[17] P. Horodecki. Separability criterion and inseparable mixed states with positive partial transposition. Physics Letters A, 9(August), 1997.

[18] M. A. Nielsen and I. L. Chuang. Quantum Computation and Quantum Information. Cambridge University Press, 2000.

[19] A. Perelomov. Generalized Coherent States and Their Applications, volume 4. SpringerVerlag, 1986.

[20] A. Peres. Separability criterion for density matrices. Physical Review Letters, 77(8):1413$1415,1996$.

[21] A. F. R. de Toledo Piza. Mecânica Quântica. Edusp, 2009.

[22] E. Schrödinger. Die gegenwärtige Situation in der Quantenmechanik. Die Naturwissenschaften, 23(48):807-812, 1935.

[23] P. W. Shor. Algorithms for quantum computation: discrete logarithms and factoring. In Proceedings 35th Annual Symposium on Foundations of Computer Science, pages 124-134. IEEE Comput. Soc. Press, 1994. 
[24] R. Simon. Peres-horodecki separability criterion for continuous variable systems. Physical review letters, 84(12):2726-9, March 2000.

[25] R. Simon, N. Mukunda, and B. Dutta. Quantum-noise matrix for multimode systems: U (n) invariance, squeezing, and normal forms. Physical Review A, 49(3):1567, 1994.

[26] E. Wigner. On the quantum correction for thermodynamic equilibrium. Physical Review, 40(5):749, 1932.

[27] P. Zanardi. Virtual Quantum Subsystems. Physical Review Letters, 87(7):4, July 2001. 